\title{
Snake venoms identification via fluorescent discrimination
}

Fei Chen, ${ }^{\S a}$ Meng Qin, ${ }^{\S b}$ Wei Liu, ${ }^{\mathrm{c}}$ Fan Wang, ${ }^{\mathrm{c}}$ Wanjie Ren, ${ }^{\mathrm{a}}$ Huihua $\mathrm{Xu}{ }^{\mathrm{a}}$ and Fengyu $\mathrm{Li}^{* \mathrm{a}}$

${ }^{a}$ College of Chemistry and Materials Science, Guangdong Provincial Key Laboratory of Functional Supramolecular Coordination Materials and Applications, Guangdong Engineering \& Technology Research Centre of Graphene-like Materials and Products, Jinan University, Guangzhou 510632, China

${ }^{b}$ College of Polymer Science and Engineering, State Key Laboratory of Polymer Materials Engineering, Sichuan University, Chengdu 610065, P. R. China

${ }^{c}$ College of Pharmacy, Xinxiang Medical University, Xinxiang 453000, China

*Email: lifengyu@jnu.edu.cn

\section{Table of Content}

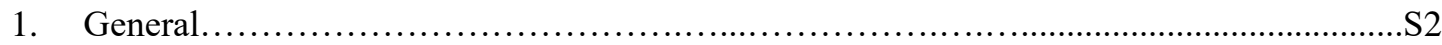

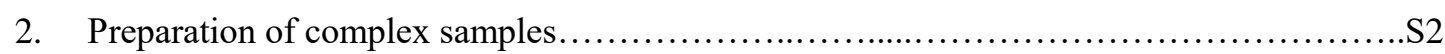

3. Operation parameters of RF-5301 spectrofluorometer for fluorescence measurements......S2

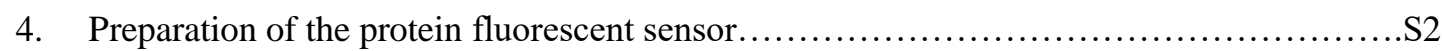

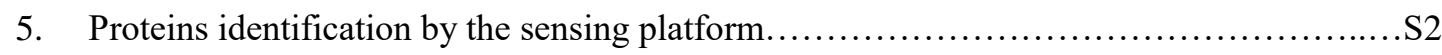

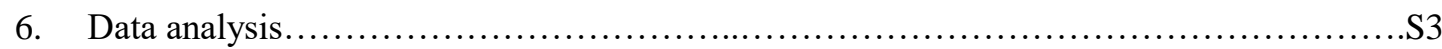

7. Molecular structures and emission spectra of four fluorescent dyes. (Figure S1-S2) ...........S4

8. Optimization of the concentration of four fluorescent dyes (Figure S3) ....................S6

9. Fluorescence responses of various dyes to eight proteins. (Figure S4-S11) ...................S7

10. LDA analysis report and results of proteins and protein mixtures. (Figure S12-S16) ........S15

11. Raw fluorescence response of the fluorescent sensor array for proteins. (Table S1) .........S20

12. Fluorescence responses of dyes to snake venoms and two interferents. (FigureS17-25) .....S30

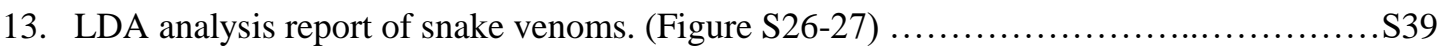

14. Raw fluorescence response of the fluorescent sensor array for seven snake venoms and interferents in PBS buffer and human plasma. (Table S2-S3) .............................................S41

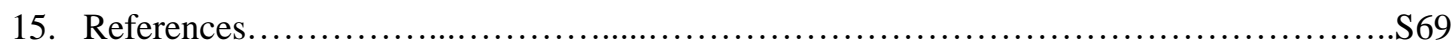




\section{- General}

Eosin Y (EY), Sulforhodamine B (SRB), and Titan yellow (TY) were purchased from Sinopharm Chemical Reagent Co., Ltd. Fluorescein isothiocyanate isomer I (FITC) and thrombin (From bovine plasma) were purchased from Beijing InnoChem Technology Co., Ltd. Phospholipase A2 from Naja atra, Phospholipase A2 from Golden Bee and Hyaluronidase were purchased from Shanghai yuanye Bio-Technology Co., Ltd. Phospholipase A2 from pig pancreas was purchased from Beijing gersion Bio-Technology Co., Ltd. Cardiotoxin and $\alpha$-Cobratoxin were purchased from Shanghai Boyao Trading Co., Ltd. Haemocoagulase was purchased from Beijing Kangchen Pharmaceutical Co., Ltd. Ophiophagus Hannah snake venom was purchased from Jiangxi Deinagkistrodon acutus Biological Technology Co., Ltd. Other snake venoms were purchased from the snake Research Institute of Yiwu City, ZheJiang Province, China. Human plasma (Heparin anticoagulant) was purchased from Guangzhou Hongquan Bio-Technology Co., Ltd. All reagents were used as received. 10mM PBS buffer (pH 7.4) was used as solvent when there no special indications. PBS solution was prepared with ultrapure water $(18.2 \mathrm{M} \Omega . \mathrm{cm})$. All proteins solutions and fluorescent dyes were freshly prepared and operated at room temperature $25^{\circ} \mathrm{C}(298 \mathrm{~K})$. Fluorescence measurements were performed by Shimadzu RF-5301 Fluorescence Spectrophotometer. UV-vis spectra were performed by Ocean Optic HR 4000 fiberoptics UV-vis spectrometer. Fluorescent images were taken by ChampChemi Professional + Image station and analyzed by software Lane 1D v4.0. (ChampChemi, Inc., china). Co., Ltd

\section{- Preparation of complex samples}

Human plasma samples

Human plasma diluted in PBS buffer at 1:100 to reduce the fluorescent background. Human plasma without added snake venom mixed with fluorescent dye was used as a control sample. Preparation of snake venom solution $(10 \mathrm{mg} / \mathrm{mL})$ with PBS buffer. Mixing $4 \mu \mathrm{L}$ of snake venoms solution $(10 \mathrm{mg} / \mathrm{mL})$ with $36 \mu \mathrm{L}$ of human plasma solution to obtain snake venom-spiked samples $(1 \mathrm{mg} / \mathrm{mL})$.

\section{- Operation parameters of RF-5301 spectrofluorometer for fluorescence measurements}

EY excitation of $520 \mathrm{~nm}$ and emission of $538 \mathrm{~nm}$ at a concentration of $8.75 \mu \mathrm{M}$. All protein solutions and Eosin $\mathrm{Y}$ mixed solution: $\lambda_{\text {ex }}=520 \mathrm{~nm} \quad \lambda_{\mathrm{em}}=525-625 \mathrm{~nm}$

FITC excitation of $496 \mathrm{~nm}$ and emission of $518 \mathrm{~nm}$ at a concentration of $1 \mu \mathrm{M}$. All protein solutions and FITC mixed solution: $\lambda_{\mathrm{ex}}=496 \mathrm{~nm} \quad \lambda_{\mathrm{em}}=500-600 \mathrm{~nm}$

SRB excitation of $583 \mathrm{~nm}$ and emission of $589 \mathrm{~nm}$ at a concentration of $25 \mu \mathrm{M}$. All protein solutions and SRB mixed solution: $\lambda_{\mathrm{ex}}=583 \mathrm{~nm} \quad \lambda_{\mathrm{em}}=500-700 \mathrm{~nm}$

TY excitation of $340 \mathrm{~nm}$ and emission of $426 \mathrm{~nm}$ at a concentration of $25 \mu \mathrm{M}$. All protein solutions and TY mixed solution: $\quad \lambda_{\mathrm{ex}}=340 \mathrm{~nm} \quad \lambda_{\mathrm{em}}=360-560 \mathrm{~nm}$

\section{- Preparation of the protein fluorescent sensor}

A 16-channel pipette was used to add $40 \mathrm{ul}$ of EY $(8.75 \mu \mathrm{M})$, FITC $(1 \mu \mathrm{M})$, SRB $(25 \mu \mathrm{M})$, and TY $(25 \mu \mathrm{M})$ to a 384-well plate, with one column of 40ul of PBS buffer or human plasma added as a control sample (Control). Then $40 \mathrm{ul}$ of proteins solution were added to the remaining column.

\section{- Proteins identification by the sensing platform}

To generate a matrix for linear discriminant analysis (LDA) and hierarchical clustering analysis (HCA), 14 parallel samples were set up for the different concentrations of various proteins, snake venoms and control.

Fluorescence responses were recorded in 6 channels (CH1: $450 \mathrm{~nm}, \mathrm{CH} 2: 480 \mathrm{~nm}, \mathrm{CH} 3: 505 \mathrm{~nm}, \mathrm{CH} 4: 535 \mathrm{~nm}$, CH5: $570 \mathrm{~nm}, \mathrm{CH6}$ : $605 \mathrm{~nm}$ ) by Fluorescence scanner (ChampChemi Professional + Image station).

\section{- Data analysis}

The statistical multivariate methods, linear discriminant analysis (LDA) was used to analyze and evaluate the response of sensors to proteins. It can more vividly explain the correlation between samples through graphic output, 
and calculate the accuracy of classification. HCA analysis is to create a hierarchical nested clustering tree by calculating the similarity between different types of data points. It can help us to analyze complex samples ${ }^{1-3}$. The LDA was carried out using SYSTAT® v13.0 and the HCA was carried out using Minitab® v16.1.1.0. 
<smiles>COc1c(Br)cc2c(-c3ccccc3C(=O)O[Na])c3cc(Br)c(=O)c(Br)c-3oc2c1Br</smiles>

Eosin Y<smiles>CCN(CC)c1ccc2c(c1)OC1=CC(=[N+](CC)CC)C=CC12c1ccc(S(=O)(=O)O)cc1S(=O)(=O)O</smiles>

SRB<smiles>O=C1OC2(c3ccc(O)cc3Oc3cc(O)ccc32)c2ccc(N=C=S)cc21</smiles>

FITC

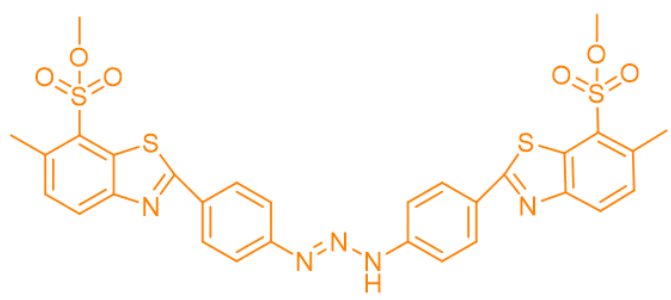

Titan yellow

1

Figure S1 Structures of fluorescent dye molecules. 
a

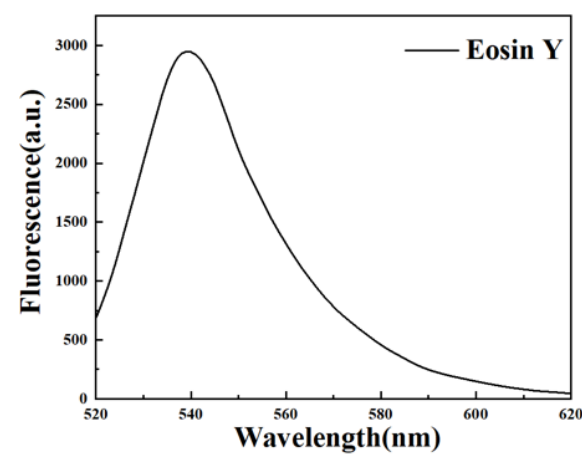

c

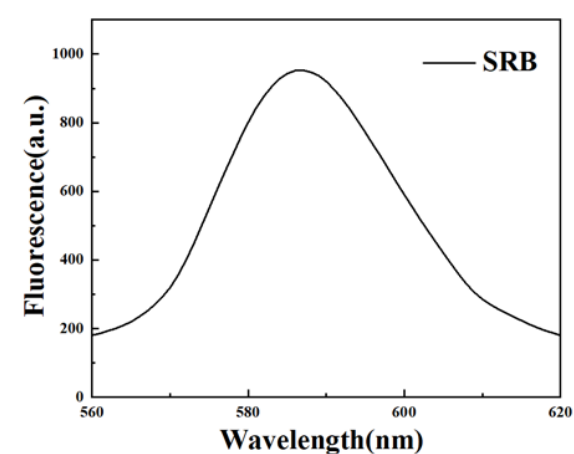

b
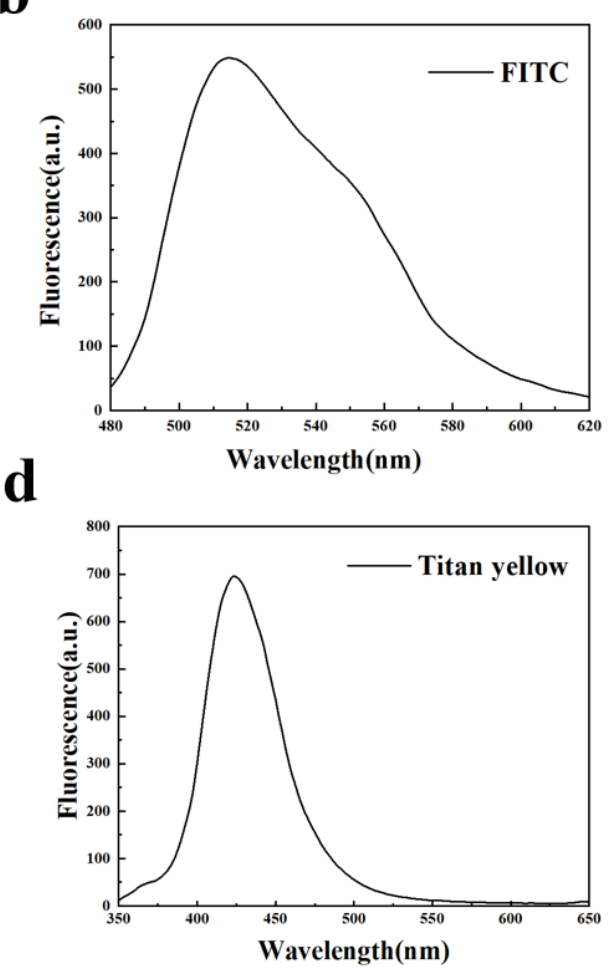

Figure S2 Fluorescence emission spectra of fluorescent dye molecules. 
a

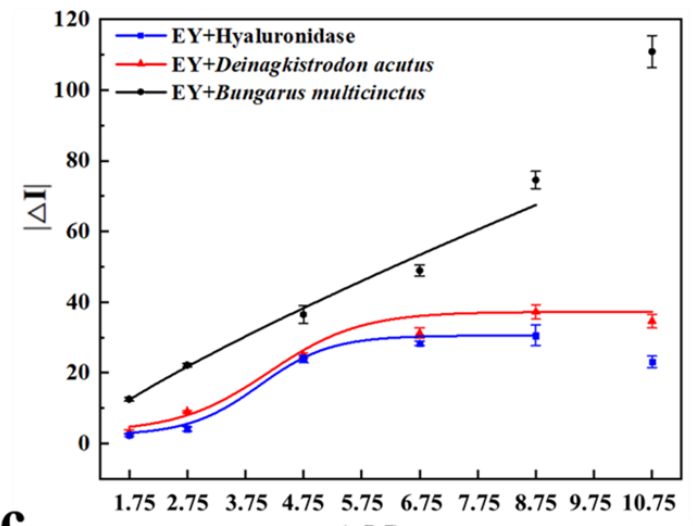

C

$[\mathrm{EY}](\mu \mathrm{M})$

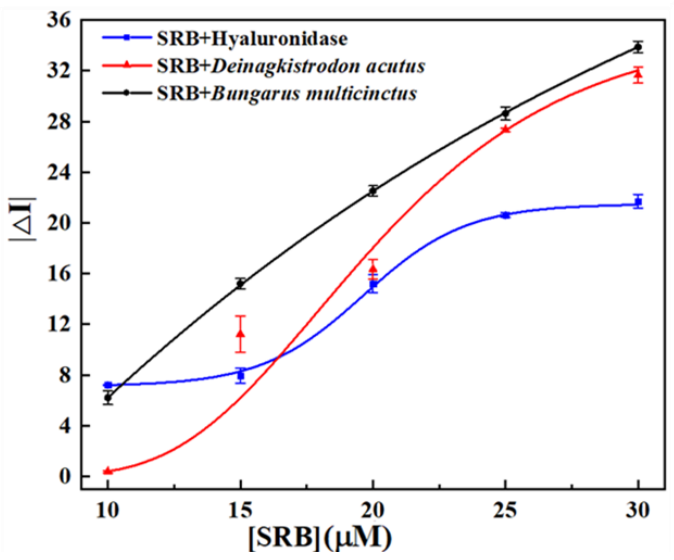

b
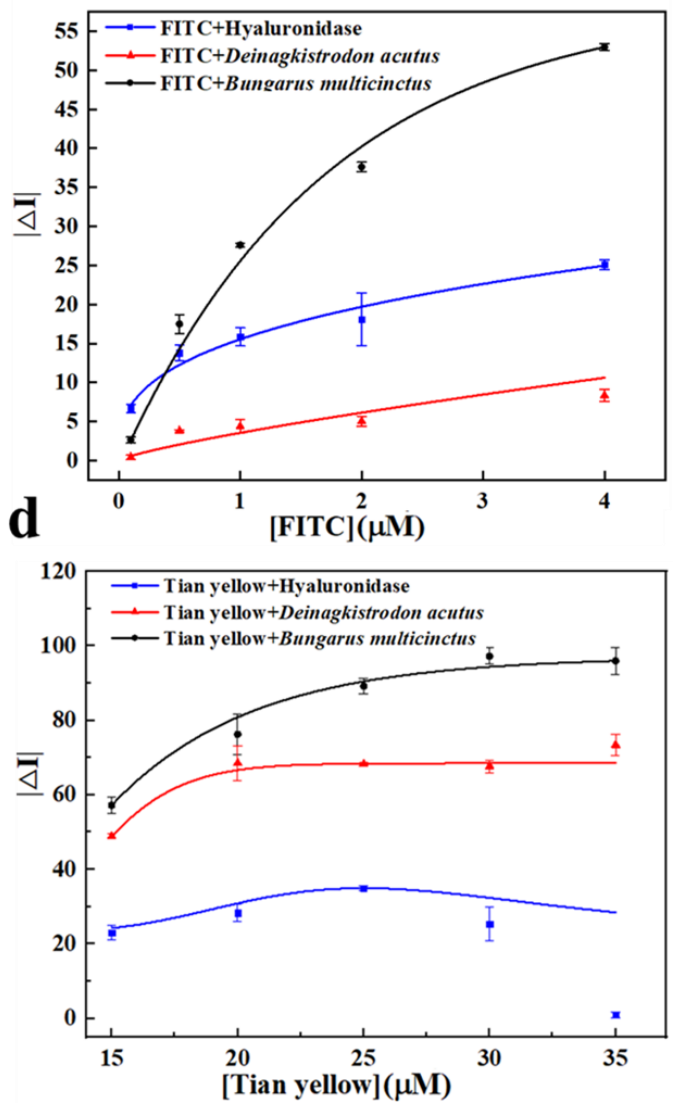

Figure S3 Fluorescent dependence to the concentrations of dyes. (EY:1.75, 2.75, 4.75, 6.75, 8.75, $10.75 \mu \mathrm{M}$; FITC: $0.1,0.5,1,2,4 \mu \mathrm{M}$; SRB: 10, 15, 20, 25, $30 \mu \mathrm{M}$; TY: 15, 20, 25, 30, $35 \mu \mathrm{M} .|\triangle \mathrm{I}|:\left|\mathrm{I}_{1}-\mathrm{I}_{0}\right|$, the modulus of the change in the fluorescence intensity $(|\triangle \mathrm{I}|)$ before $\left(\mathrm{I}_{0}\right)$ and after $\left(\mathrm{I}_{1}\right)$ binding with protein.) 
a

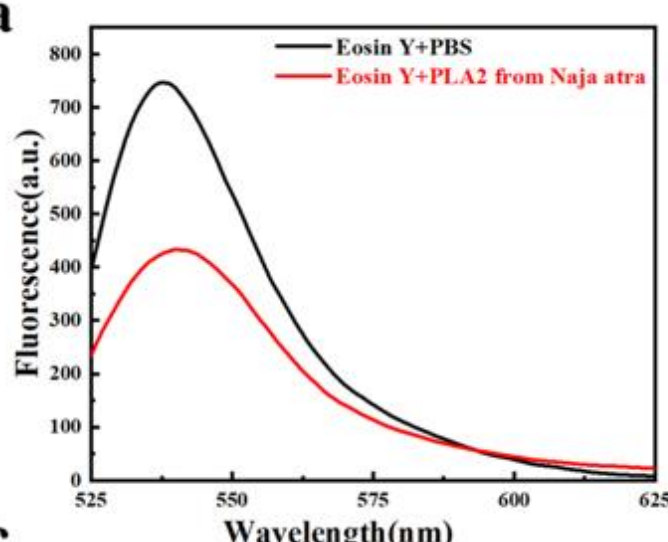

c

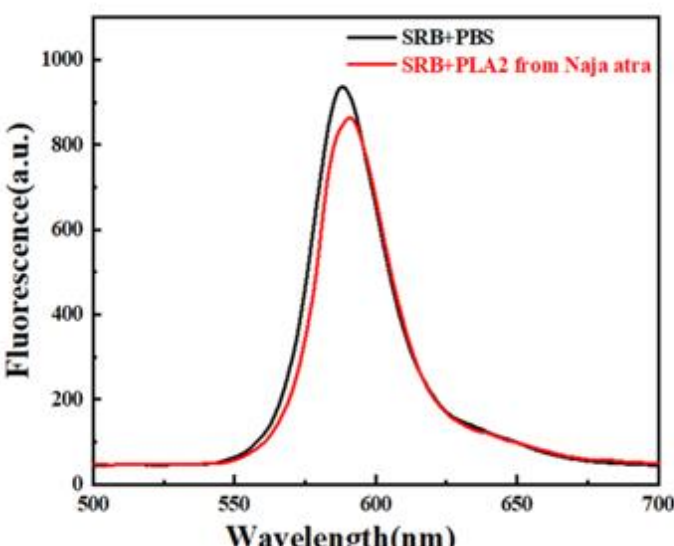

b
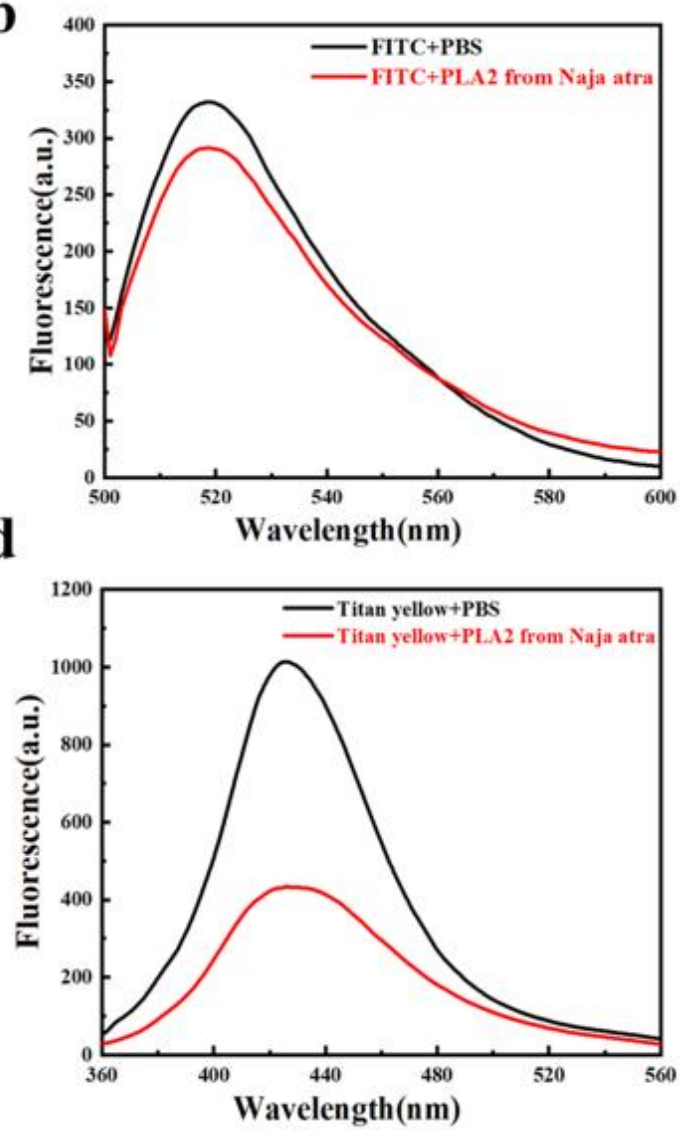

Figure S4 Fluorescence responses of various dyes to PLA2 from Naja atra. 
a

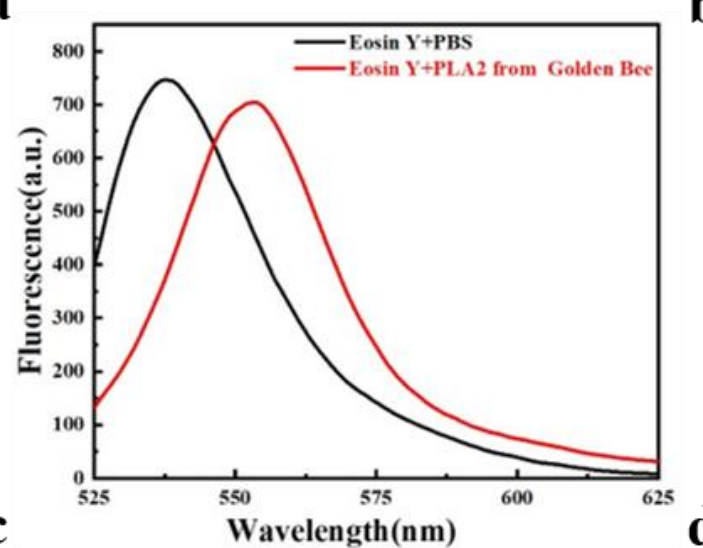

c

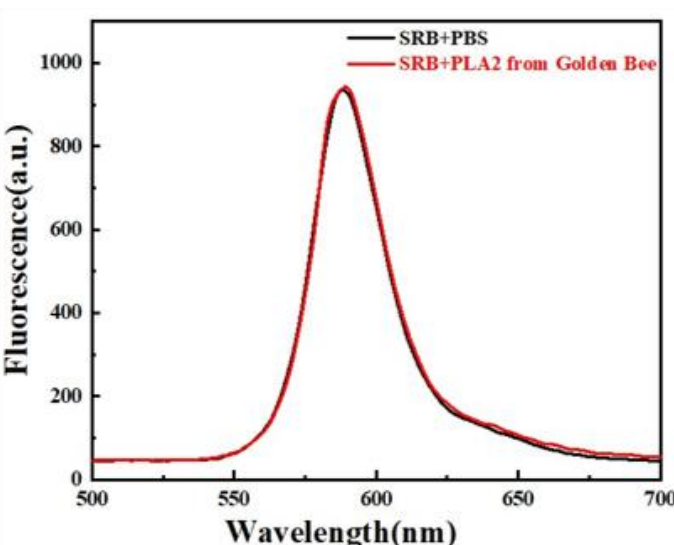

b
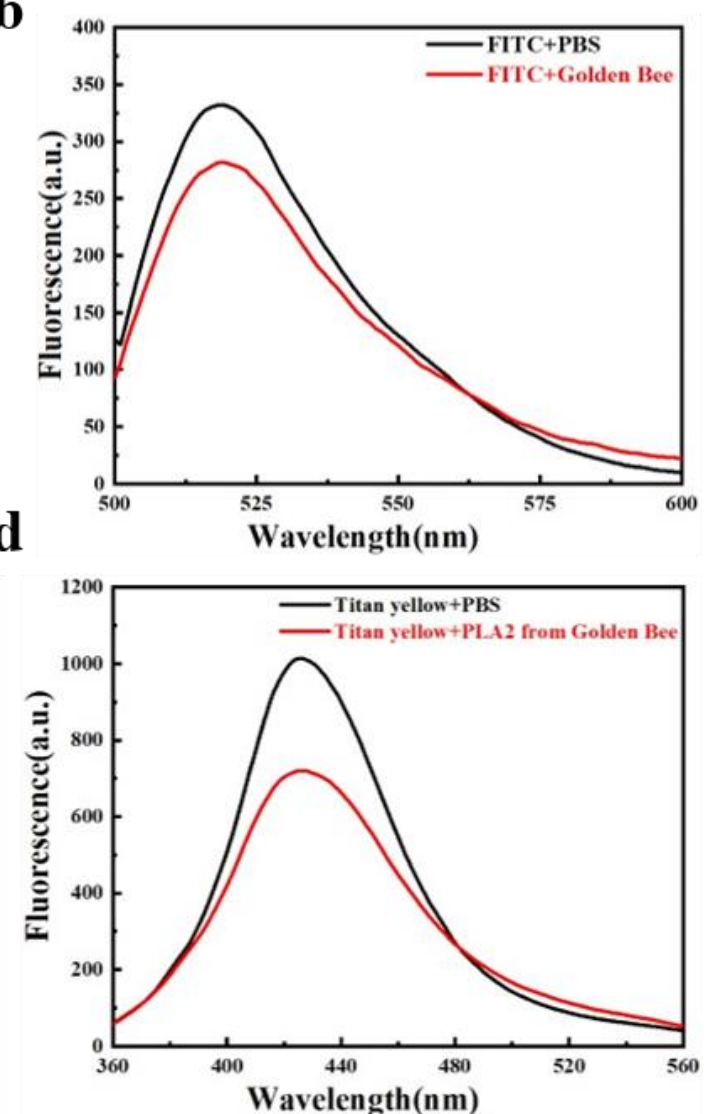

Figure S5 Fluorescence responses of various dyes to PLA2 from Golden Bee. 
a

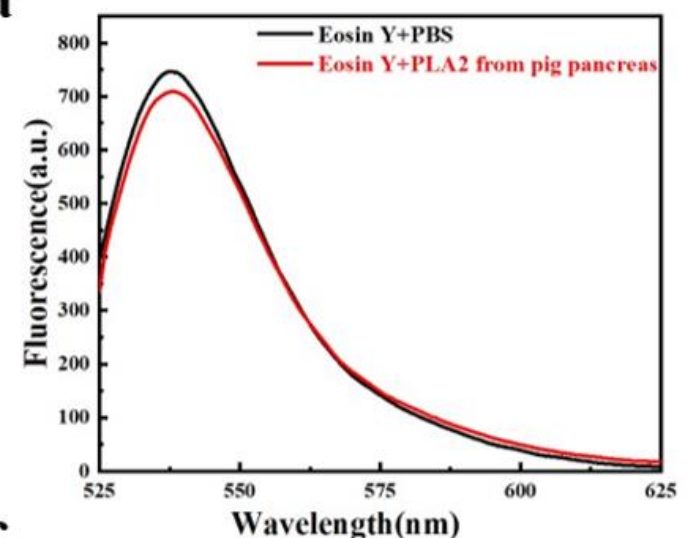

c

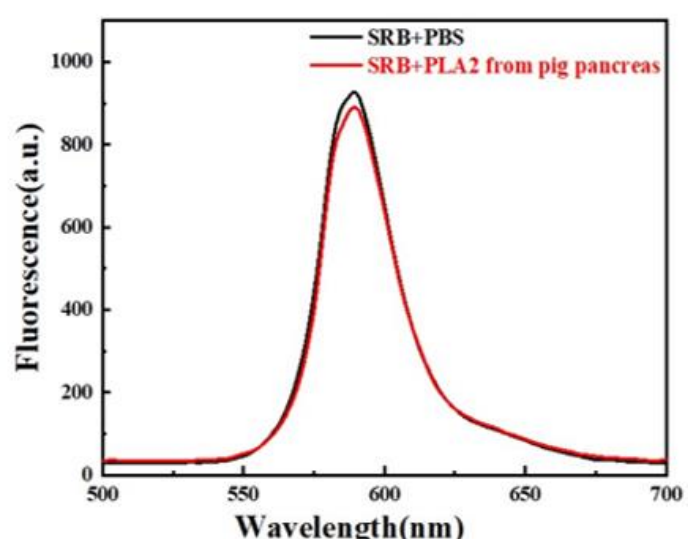

b
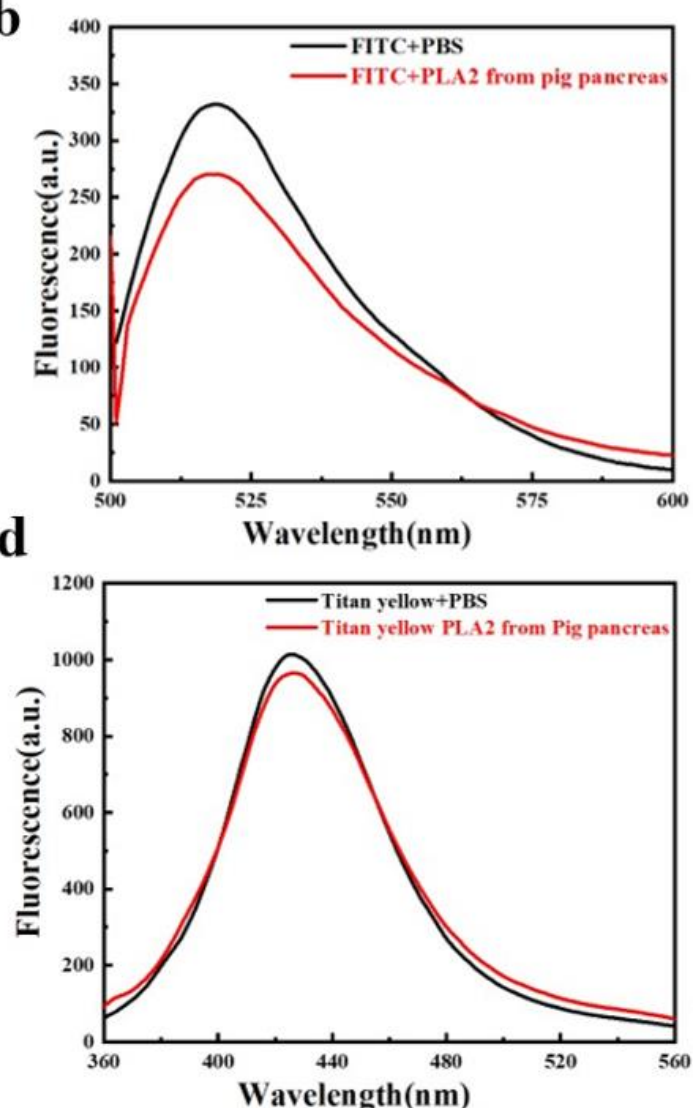

Figure S6 Fluorescence responses of various dyes to PLA2 from pig pancreas. 
a

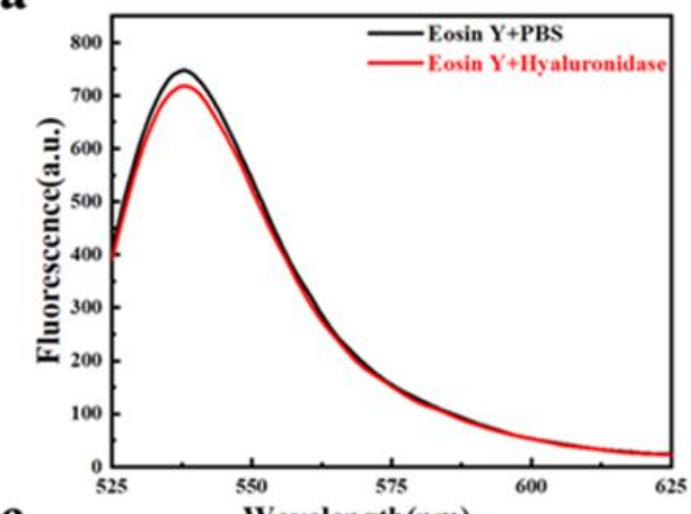

c

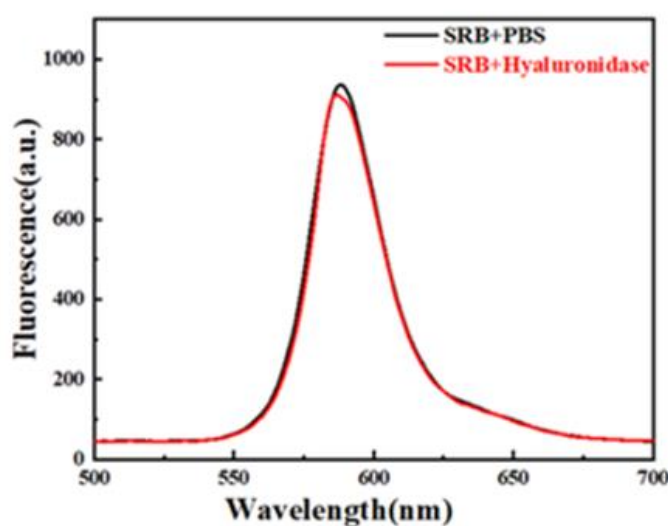

b
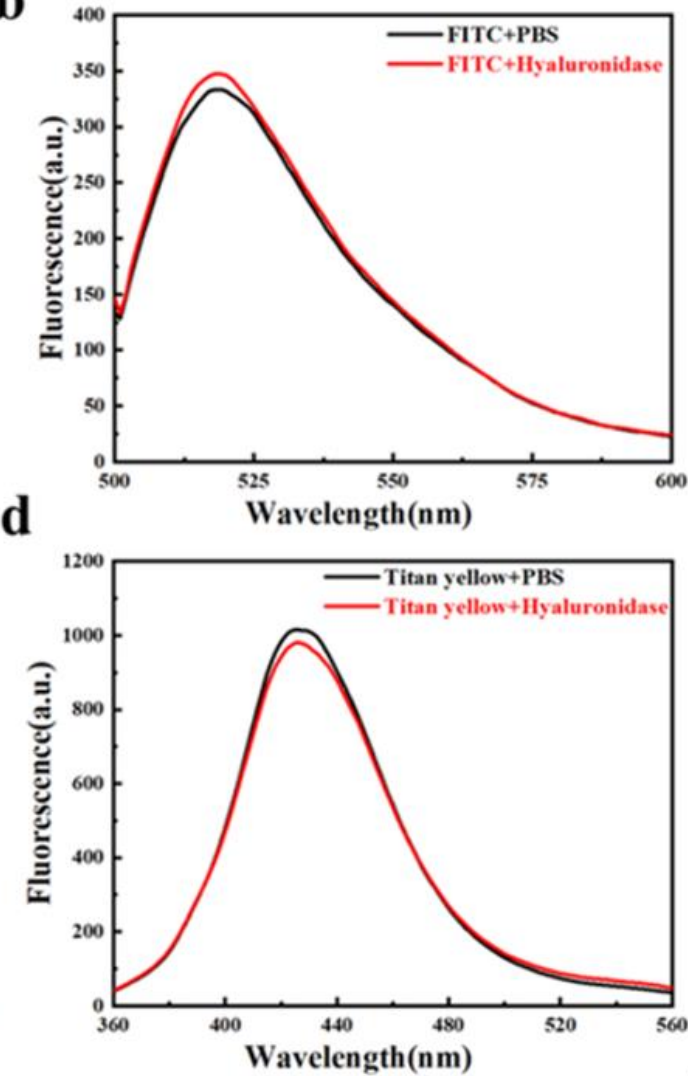

Figure S7 Fluorescence responses of various dyes to hyaluronidase. 

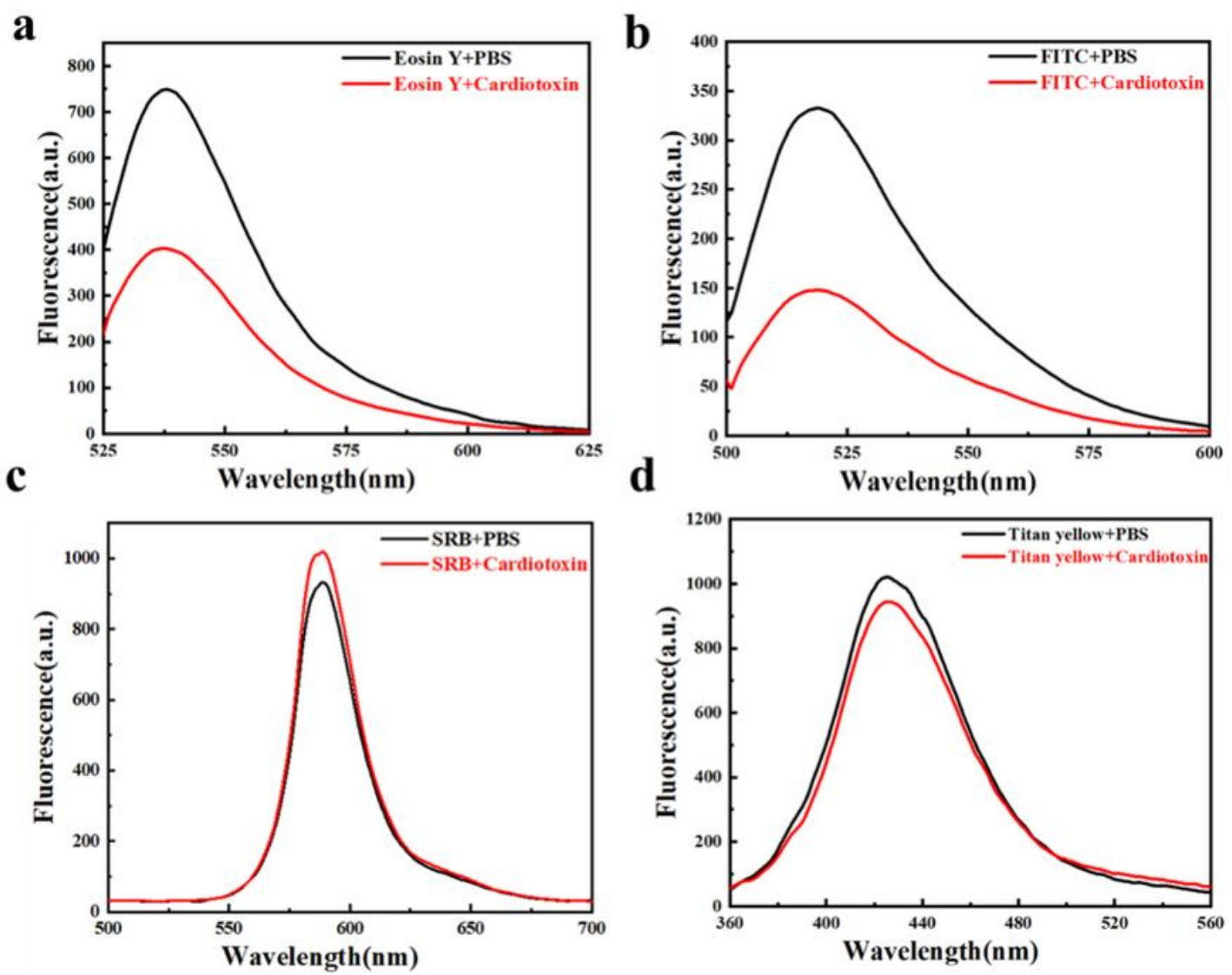

Figure S8 Fluorescence responses of various dyes to cardiotoxin. 

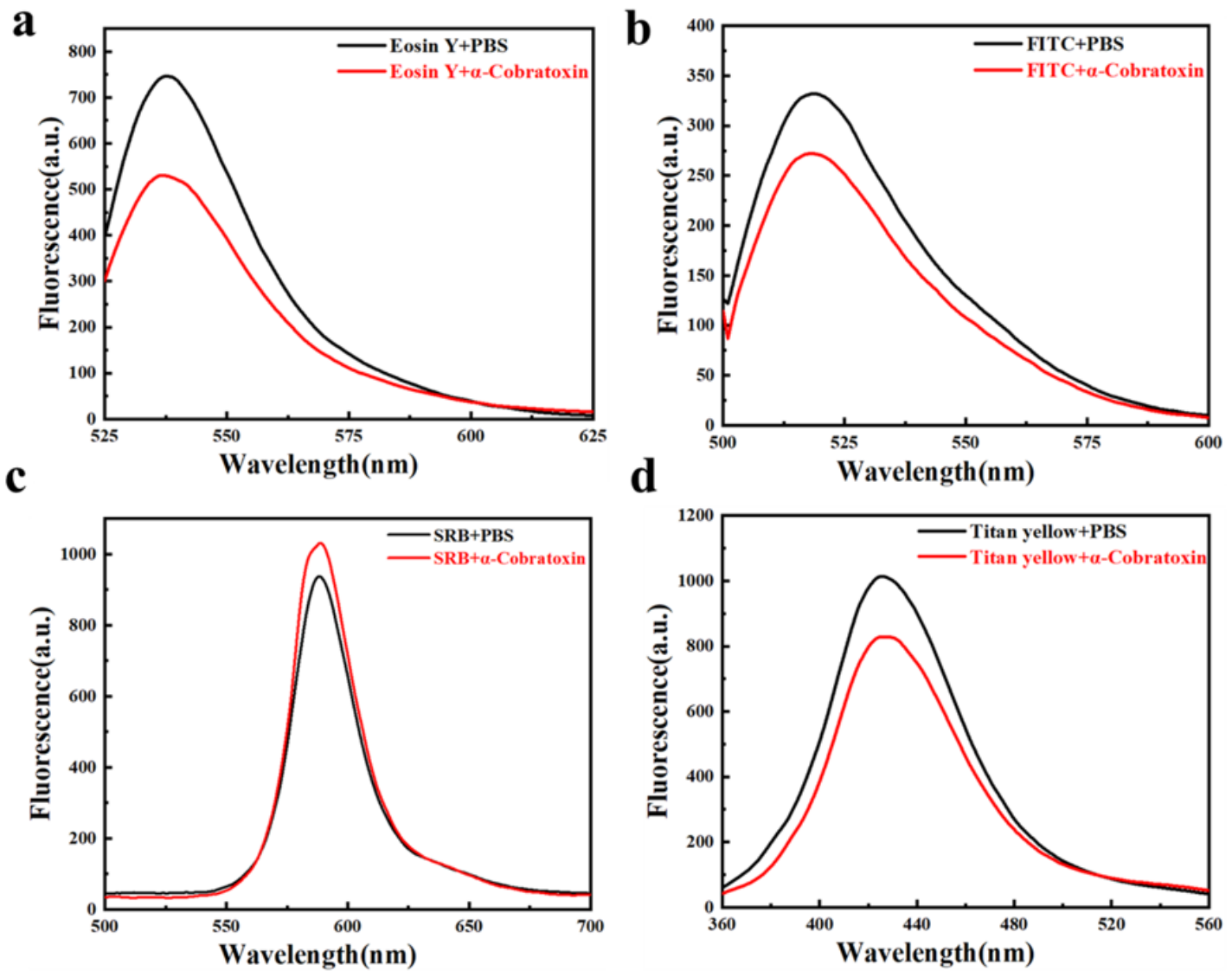

20 Figure S9 Fluorescence responses of various dyes to $\alpha$-Cobratoxin. 
a

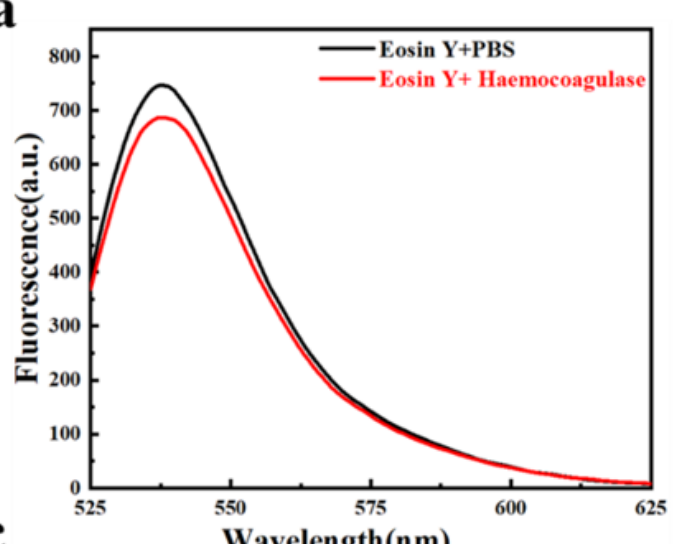

c

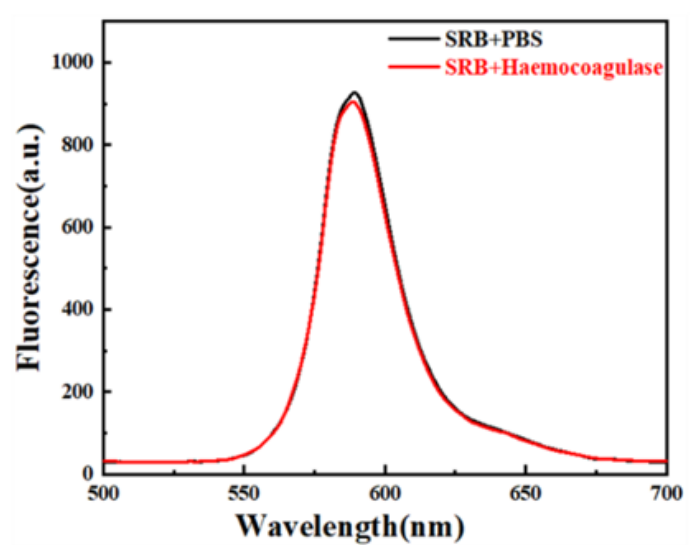

b
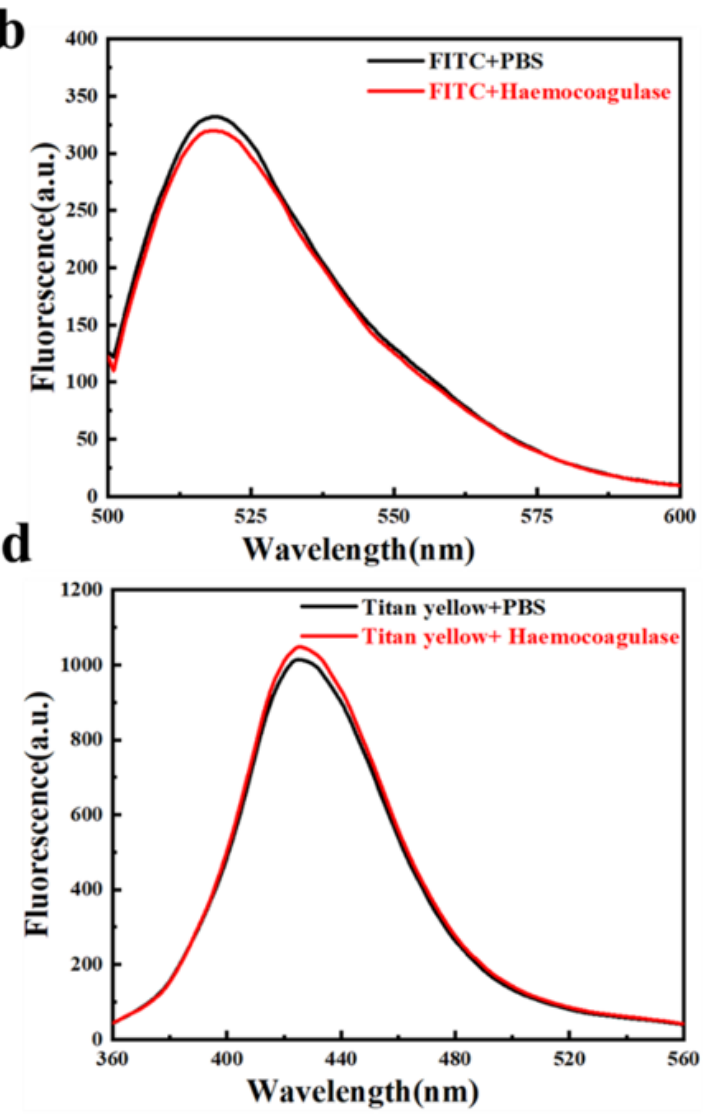

Figure S10 Fluorescence responses of various dyes to haemocoagulase. 

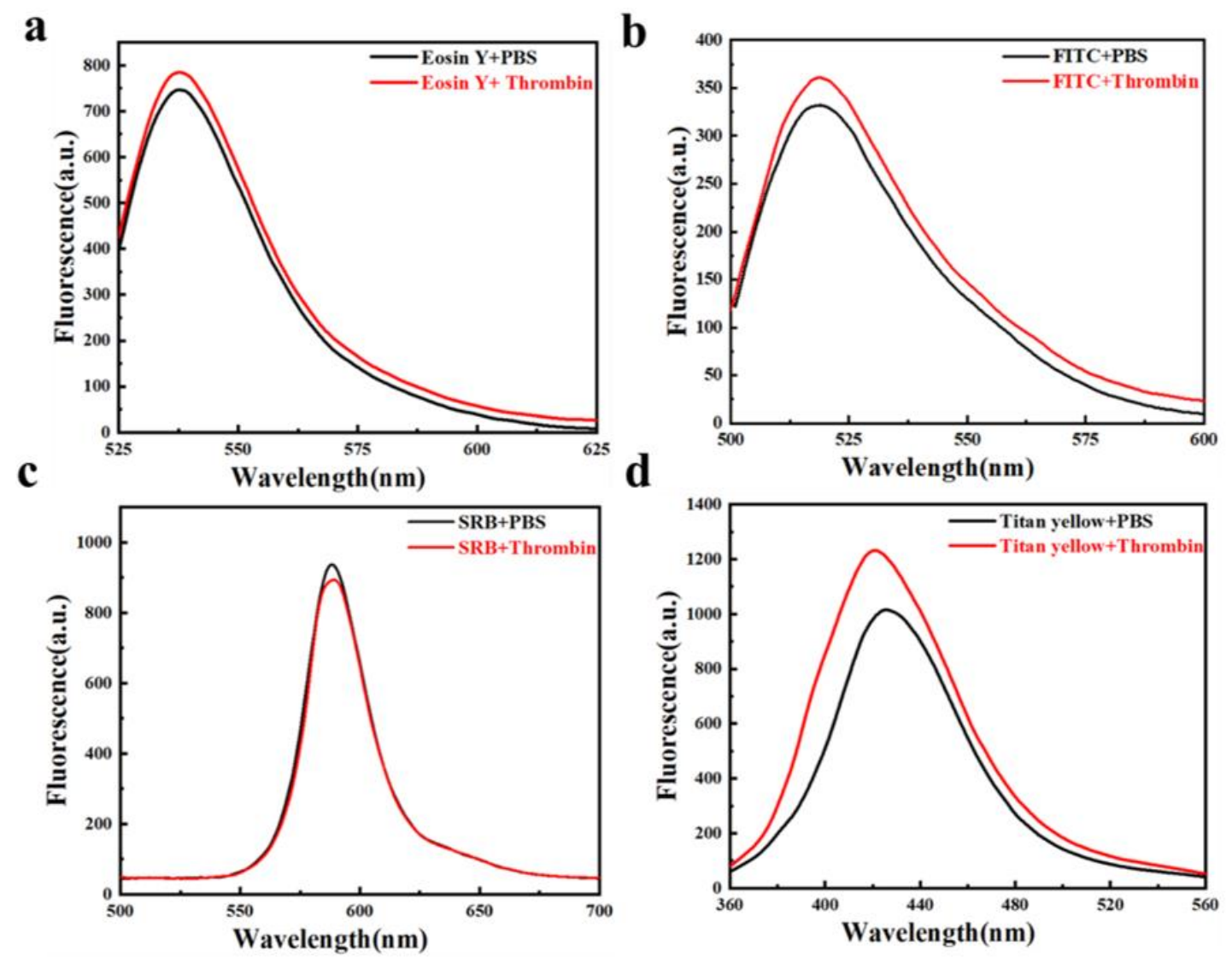

24 Figure S11 Fluorescence responses of various dyes to thrombin. 


Jackknifed Classification Matrix
\begin{tabular}{|l|r|r|r|r|r|r|r|r|}
\hline & Cardiotoxin & Control & Haemocoagulase & Hyaluronidase & PhospholipaseA2 & Thrombin & a-Cobratoxin & $\%$ correct \\
\hline Cardiotoxin & 14 & 0 & 0 & 0 & 0 & 0 & 0 & 100 \\
\hline Control & 0 & 14 & 0 & 0 & 0 & 0 & 0 & 100 \\
\hline Haemocoagulase & 0 & 0 & 14 & 0 & 0 & 0 & 0 & 100 \\
\hline Hyaluronidase & 0 & 0 & 0 & 14 & 0 & 0 & 0 & 100 \\
\hline PhospholipaseA2 & 0 & 0 & 0 & 0 & 14 & 0 & 0 & 100 \\
\hline Thrombin & 0 & 0 & 0 & 0 & 0 & 14 & 0 & 100 \\
\hline a-Cobratoxin & 0 & 0 & 0 & 0 & 0 & 0 & 14 & 100 \\
\hline Total & 14 & 14 & 14 & 14 & 14 & 14 & 14 & 100 \\
\hline
\end{tabular}

b

Canonical Scores Plot

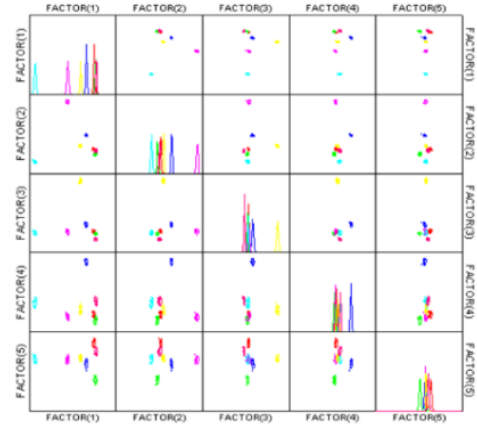

$\operatorname{VAR} \$(1)$

\section{$\square$ Cardiotoxin}

Control

Haemocoagulase

Hyaluronidase

PhospholipaseA2

Thrombin

$\square$ a-Cobratoxin

Figure S12 (a) Jackknifed classification matrix of the fluorescent sensor array for the discrimination of different proteins in PBS buffer. (PhospholipaseA2 from Naja atra) (b) Canonical scores plot of the fluorescent sensor array for the discrimination of different proteins in PBS buffer. 


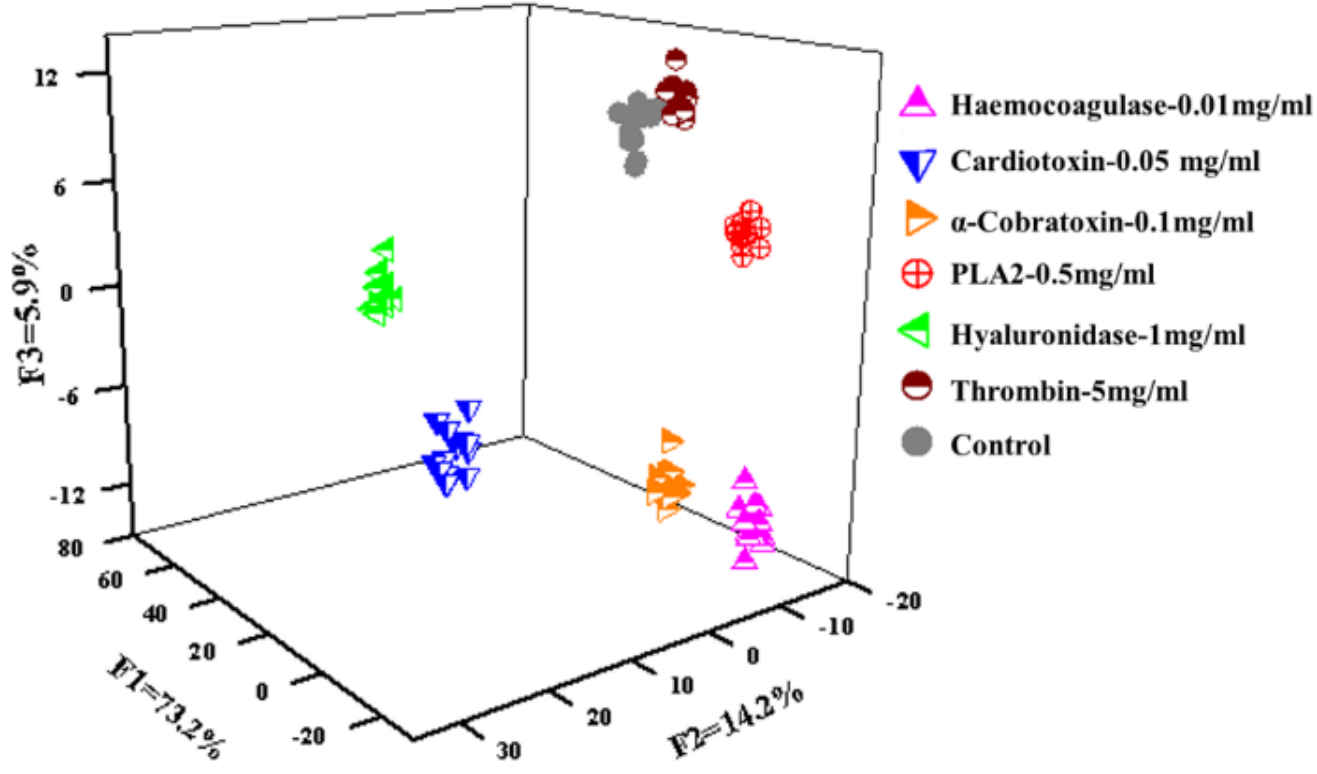

Figure S13 LDA results of different proteins with different concentrations in PBS buffer. (Haemocoagulase in 0.01 $\mathrm{mg} / \mathrm{mL}$, Cardiotoxin in $0.05 \mathrm{mg} / \mathrm{mL}, \alpha$-Cobratoxin in $0.1 \mathrm{mg} / \mathrm{mL}$, PLA2 from Naja atra in $0.5 \mathrm{mg} / \mathrm{mL}$, Hyaluronidase in $1 \mathrm{mg} / \mathrm{mL}$, Thrombin in $5 \mathrm{mg} / \mathrm{mL}$ ). 


\section{a}

Jackknifed Classification Matrix
\begin{tabular}{|l|r|r|r|r|r|r|r|r|}
\hline & A & B & C & D & E & F & G & \%correct \\
\hline A & 14 & 0 & 0 & 0 & 0 & 0 & 0 & 100 \\
\hline B & 0 & 14 & 0 & 0 & 0 & 0 & 0 & 100 \\
\hline C & 0 & 0 & 14 & 0 & 0 & 0 & 0 & 100 \\
\hline D & 0 & 0 & 0 & 14 & 0 & 0 & 0 & 100 \\
\hline E & 0 & 0 & 0 & 0 & 14 & 0 & 0 & 100 \\
\hline F & 0 & 0 & 0 & 0 & 0 & 14 & 0 & 100 \\
\hline G & 0 & 0 & 0 & 0 & 0 & 0 & 14 & 100 \\
\hline Total & 14 & 14 & 14 & 14 & 14 & 14 & 14 & 100 \\
\hline
\end{tabular}

b

Canonical Scores Plot

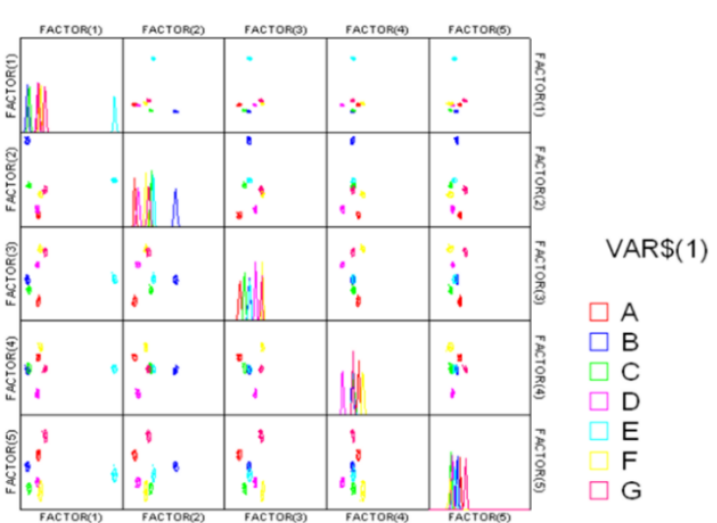

Figure S14 (a) Jackknifed classification matrix of the fluorescent sensor array for the discrimination of proteins with different concentrations in PBS buffer. (b) Canonical scores plot of the fluorescent sensor array for the discrimination of proteins with different concentrations in PBS buffer. (A: Haemocoagulase in 0.01; mg/mL; B: Cardiotoxin in 0.05 $\mathrm{mg} / \mathrm{mL}$; C: $\alpha$-cobratoxin in $0.1 \mathrm{mg} / \mathrm{mL}$; D: PLA2 from Naja atra in $0.5 \mathrm{mg} / \mathrm{mL}$ E: Hyaluronidase in $1 \mathrm{mg} / \mathrm{mL} \mathrm{F}$ : Thrombin in $5 \mathrm{mg} / \mathrm{mL}$ G: Control). 


\section{a}

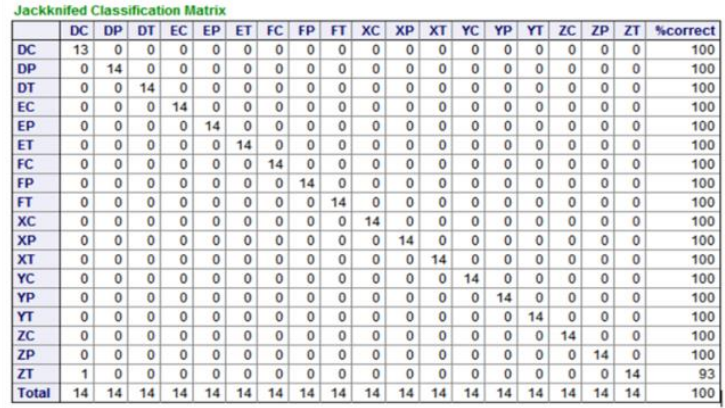

b

Canonical Scores Plot

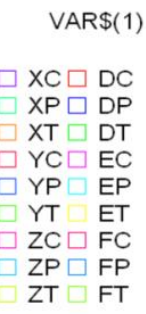

Figure S15 (a) Jackknifed classification matrix of the fluorescent sensor array for the discrimination of three proteins with different concentration gradients in PBS buffer. (b) Canonical scores plot of the fluorescent sensor array for the discrimination of three proteins with different concentration gradients in PBS buffer.

(T: Thrombin, C: $\alpha$-Cobratoxin P: PLA2 from Naja atra; D: $0.01 \mathrm{mg} / \mathrm{mL}$, E: $0.02 \mathrm{mg} / \mathrm{mL}, \mathrm{F}: 0.05 \mathrm{mg} / \mathrm{mL}, \mathrm{X}: 0.1$ $\mathrm{mg} / \mathrm{mL}, \mathrm{Y}: 0.2 \mathrm{mg} / \mathrm{mL}, \mathrm{Z}: 0.5 \mathrm{mg} / \mathrm{mL}$; DT, ET, FT, XT, YT, ZT are thrombin with concentrations of 0.01, 0.02, 0.05, $0.1,0.2,0.5 \mathrm{mg} / \mathrm{mL}$, respectively. DC, EC, FC, XC, YC, ZC are $\alpha$-Cobratoxin with concentrations of $0.01,0.02$, $0.05,0.1,0.2,0.5 \mathrm{mg} / \mathrm{mL}$, respectively. DP, EP, FP, XP, YP, ZP are PLA2 from Naja atra with concentrations of $0.01,0.02,0.05,0.1,0.2,0.5 \mathrm{mg} / \mathrm{mL}$, respectively.) 
a

Jackknifed Classification Matrix
\begin{tabular}{|l|r|r|r|r|r|r|r|r|r|}
\hline & A & B & C & D & E & F & G & H & $\%$ correct \\
\hline A & 14 & 0 & 0 & 0 & 0 & 0 & 0 & 0 & 100 \\
\hline B & 0 & 14 & 0 & 0 & 0 & 0 & 0 & 0 & 100 \\
\hline C & 0 & 0 & 14 & 0 & 0 & 0 & 0 & 0 & 100 \\
\hline D & 0 & 0 & 0 & 14 & 0 & 0 & 0 & 0 & 100 \\
\hline E & 0 & 0 & 0 & 0 & 14 & 0 & 0 & 0 & 100 \\
\hline F & 0 & 0 & 0 & 0 & 0 & 14 & 0 & 0 & 100 \\
\hline G & 0 & 0 & 0 & 0 & 0 & 0 & 14 & 0 & 100 \\
\hline H & 0 & 0 & 0 & 0 & 0 & 0 & 0 & 14 & 100 \\
\hline Total & 14 & 14 & 14 & 14 & 14 & 14 & 14 & 14 & 100 \\
\hline
\end{tabular}

b

Canonical Scores Plot

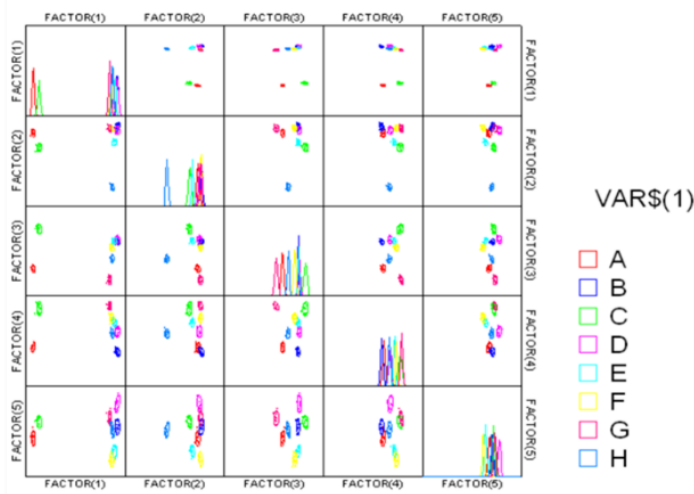

Figure S16 (a) Jackknifed classification matrix of the fluorescent sensor array for the discrimination of protein mixtures in PBS buffer. (b) Canonical scores plot of the fluorescent sensor array for the discrimination of protein mixtures in PBS buffer. (A: a mixture of hyaluronidase, cardiotoxin and $\alpha$-Cobratoxin; B: a mixture of PLA2 from Naja atra, haemocoagulase and thrombin; C: a mixture of hyaluronidase, cardiotoxin and $\alpha$-Cobratoxin; D: a mixture of PLA2 from Naja atra and $\alpha$-Cobratoxin; E: a mixture of haemocoagulase and thrombin; F: a mixture of $\alpha$ Cobratoxin and haemocoagulase; G: a mixture of $\alpha$-Cobratoxin and thrombin; H: PLA2 from Naja atra) 
Table S1 Raw fluorescence responses in six channels of the fluorescent sensor array in the discrimination of six 56 proteins in PBS buffer.

\begin{tabular}{|c|c|c|c|c|c|c|c|c|c|c|c|c|}
\hline & \multicolumn{12}{|c|}{ Eosin $Y$} \\
\hline & CH1 & $\mathrm{CH} 2$ & $\mathrm{CH} 3$ & $\mathrm{CH} 4$ & CH5 & CH6 & CH1 & $\mathrm{CH} 2$ & $\mathrm{CH} 3$ & $\mathrm{CH} 4$ & CH5 & CH6 \\
\hline & 302 & 302 & 302 & 302 & 302 & 302 & 365 & 365 & 365 & 365 & 365 & 365 \\
\hline & 450 & 480 & 505 & 535 & 570 & 605 & 450 & 480 & 505 & 535 & 570 & 605 \\
\hline \multirow{14}{*}{$\begin{array}{c}\text { PLA2 from } \\
\text { Naja atra }\end{array}$} & 4714 & 6447 & 6470 & 16515 & 13413 & 13506 & 3238 & 7649 & 2172 & 12959 & 7818 & 6309 \\
\hline & 4832 & 5903 & 7266 & 18166 & 13111 & 13049 & 3242 & 7674 & 2251 & 12933 & 7534 & 6376 \\
\hline & 4944 & 6822 & 6316 & 18036 & 13353 & 12647 & 3301 & 6602 & 2464 & 12070 & 7583 & 6569 \\
\hline & 4949 & 6375 & 7206 & 17533 & 13977 & 12913 & 3536 & 6823 & 2675 & 12260 & 7219 & 6674 \\
\hline & 5500 & 7211 & 6767 & 17717 & 14618 & 13601 & 3684 & 6932 & 2785 & 12093 & 7750 & 6372 \\
\hline & 4936 & 7419 & 6724 & 17671 & 15384 & 14099 & 3661 & 7934 & 2896 & 12217 & 7965 & 6771 \\
\hline & 5210 & 6978 & 7260 & 16789 & 15108 & 14015 & 3865 & 7258 & 2570 & 11524 & 7839 & 6817 \\
\hline & 5273 & 7367 & 6769 & 16544 & 15844 & 14424 & 3962 & 7034 & 2768 & 11880 & 8040 & 6211 \\
\hline & 5161 & 6580 & 6306 & 17580 & 13999 & 13356 & 3751 & 7118 & 2716 & 10335 & 7270 & 6946 \\
\hline & 5185 & 6398 & 6341 & 17916 & 12751 & 13287 & 3866 & 6938 & 2664 & 11450 & 7564 & 6716 \\
\hline & 5446 & 6986 & 6470 & 16880 & 12974 & 13135 & 3876 & 7442 & 2565 & 11924 & 7437 & 6837 \\
\hline & 5350 & 6694 & 7312 & 17193 & 12724 & 14347 & 3802 & 7014 & 2522 & 11820 & 7567 & 6243 \\
\hline & 5368 & 7118 & 7232 & 17373 & 12844 & 11810 & 3660 & 7390 & 2827 & 13257 & 7496 & 6826 \\
\hline & 5215 & 7490 & 7356 & 17557 & 14379 & 13852 & 3781 & 6573 & 2600 & 11797 & 7351 & 6234 \\
\hline \multirow{14}{*}{ Hyaluronidase } & 4265 & 7719 & 5015 & 12707 & 10041 & 9377 & 4556 & 5826 & 4206 & 10123 & 7757 & 4745 \\
\hline & 4486 & 7660 & 4874 & 11590 & 9519 & 9317 & 3998 & 5562 & 4541 & 10104 & 6959 & 4607 \\
\hline & 4392 & 7316 & 4607 & 10523 & 8841 & 9230 & 4179 & 5789 & 4179 & 9463 & 6740 & 4396 \\
\hline & 4566 & 7586 & 4747 & 10964 & 9916 & 8936 & 4267 & 6140 & 4525 & 9455 & 6885 & 4472 \\
\hline & 4621 & 7965 & 5141 & 11538 & 10044 & 9351 & 4233 & 5869 & 4624 & 9740 & 7151 & 4574 \\
\hline & 4777 & 7768 & 5352 & 11703 & 9929 & 9800 & 4187 & 5118 & 4937 & 10692 & 7359 & 4976 \\
\hline & 4641 & 7978 & 5335 & 12009 & 10310 & 9645 & 4412 & 5672 & 4959 & 10091 & 7225 & 4723 \\
\hline & 4711 & 7492 & 5201 & 11543 & 9639 & 9171 & 4251 & 5698 & 5006 & 10399 & 7458 & 4635 \\
\hline & 4654 & 7477 & 5125 & 11253 & 9308 & 9008 & 4317 & 5144 & 4845 & 10879 & 7494 & 4884 \\
\hline & 4369 & 7604 & 4709 & 11418 & 9303 & 8968 & 4241 & 5882 & 4684 & 10766 & 7126 & 4705 \\
\hline & 4596 & 6943 & 4729 & 11038 & 9252 & 9087 & 4186 & 6059 & 4566 & 10387 & 7254 & 4610 \\
\hline & 4549 & 7357 & 5228 & 12656 & 8821 & 10417 & 4235 & 6127 & 4642 & 10527 & 7735 & 4954 \\
\hline & 4202 & 7261 & 4915 & 10789 & 9741 & 8929 & 4396 & 5235 & 4355 & 9282 & 7137 & 4350 \\
\hline & 4302 & 8195 & 4986 & 12710 & 9677 & 10229 & 4401 & 5946 & 4868 & 10070 & 7571 & 4875 \\
\hline \multirow{8}{*}{ Cardiotoxin } & 4469 & 5709 & 5046 & 7962 & 5078 & 5393 & 3441 & 6009 & 2426 & 6547 & 3152 & 2634 \\
\hline & 4677 & 7007 & 5148 & 7193 & 5400 & 4563 & 3108 & 6410 & 2507 & 6664 & 3196 & 2054 \\
\hline & 4144 & 6216 & 5250 & 6791 & 4748 & 4358 & 3252 & 5571 & 2678 & 6236 & 3229 & 2082 \\
\hline & 4309 & 6636 & 5047 & 8570 & 5351 & 4318 & 3210 & 6027 & 2810 & 6180 & 3159 & 2189 \\
\hline & 4315 & 6897 & 5477 & 8551 & 5250 & 4755 & 3050 & 5283 & 3024 & 5996 & 3113 & 2468 \\
\hline & 4759 & 7096 & 5526 & 7548 & 5000 & 4949 & 3403 & 5648 & 3064 & 6087 & 3011 & 2445 \\
\hline & 4460 & 7062 & 5457 & 8493 & 5765 & 5216 & 3635 & 5938 & 2740 & 5959 & 3165 & 2686 \\
\hline & 4383 & 6364 & 5430 & 8222 & 5585 & 5214 & 3413 & 5930 & 2985 & 5713 & 3156 & 2563 \\
\hline
\end{tabular}




\begin{tabular}{|c|c|c|c|c|c|c|c|c|c|c|c|c|}
\hline & 4620 & 6901 & 5520 & 7571 & 5070 & 4704 & 3307 & 5704 & 2802 & 5982 & 3054 & 2759 \\
\hline & 4091 & 6784 & 5001 & 7593 & 5022 & 4846 & 3144 & 5952 & 3084 & 5870 & 3248 & 2783 \\
\hline & 4326 & 6482 & 5640 & 8216 & 5195 & 4817 & 3226 & 5314 & 2881 & 5690 & 3323 & 2815 \\
\hline & 4065 & 6266 & 5078 & 8517 & 5610 & 4964 & 3094 & 5400 & 3166 & 6109 & 3349 & 2962 \\
\hline & 4626 & 6712 & 6138 & 8136 & 5836 & 5315 & 3085 & 5950 & 2929 & 6244 & 3057 & 2655 \\
\hline & 4470 & 7048 & 6239 & 7293 & 5879 & 5446 & 3571 & 5304 & 3081 & 6166 & 3389 & 2421 \\
\hline \multirow{14}{*}{$\alpha$-Cobratoxin } & 4897 & 6296 & 5245 & 7353 & 5310 & 4484 & 3295 & 5753 & 2839 & 4391 & 2615 & 2392 \\
\hline & 5264 & 6222 & 4682 & 6715 & 4453 & 4216 & 3030 & 5854 & 2518 & 4756 & 2558 & 2148 \\
\hline & 4648 & 6084 & 4907 & 6538 & 4077 & 4219 & 3201 & 5425 & 2534 & 5411 & 2684 & 2092 \\
\hline & 5142 & 6887 & 4919 & 6654 & 4534 & 4317 & 2978 & 5115 & 2616 & 4362 & 2453 & 2106 \\
\hline & 4776 & 6960 & 5187 & 7611 & 5005 & 5003 & 2924 & 5660 & 2713 & 4416 & 2447 & 2216 \\
\hline & 4758 & 6762 & 5287 & 7486 & 5159 & 4909 & 2906 & 5643 & 2915 & 4769 & 2922 & 2277 \\
\hline & 4778 & 6095 & 4944 & 7339 & 5157 & 5190 & 3115 & 5535 & 2919 & 4559 & 2903 & 2368 \\
\hline & 4960 & 6626 & 5438 & 7285 & 4965 & 4942 & 3173 & 5262 & 2704 & 4753 & 2508 & 2570 \\
\hline & 5167 & 6319 & 5215 & 7508 & 4591 & 4368 & 2809 & 5258 & 2982 & 4414 & 2761 & 2234 \\
\hline & 4833 & 6702 & 5256 & 7316 & 4859 & 4342 & 3075 & 5915 & 2736 & 4679 & 2471 & 2659 \\
\hline & 5157 & 6259 & 5034 & 7145 & 5193 & 4218 & 3217 & 5871 & 2757 & 4812 & 2647 & 2518 \\
\hline & 5130 & 6931 & 5565 & 8088 & 4737 & 4273 & 3068 & 5595 & 2588 & 4891 & 2704 & 2538 \\
\hline & 4784 & 6957 & 4897 & 7443 & 5495 & 5143 & 3182 & 5073 & 2964 & 5066 & 2969 & 2615 \\
\hline & 4830 & 6866 & 5148 & 8027 & 5246 & 4641 & 3669 & 5138 & 2569 & 4511 & 3091 & 2366 \\
\hline \multirow{14}{*}{ Haemocoagulase } & 4105 & 6653 & 4389 & 6312 & 4517 & 4523 & 3316 & 4665 & 2729 & 4755 & 2655 & 2561 \\
\hline & 3776 & 5969 & 4455 & 6392 & 4042 & 4171 & 2999 & 4381 & 3018 & 4828 & 2742 & 2229 \\
\hline & 4156 & 6646 & 4849 & 6201 & 4017 & 4193 & 2952 & 4684 & 2794 & 4515 & 2588 & 2391 \\
\hline & 3926 & 6554 & 4932 & 7182 & 4822 & 4430 & 2845 & 4717 & 2854 & 4456 & 2489 & 2279 \\
\hline & 3990 & 6283 & 4858 & 7015 & 4834 & 5028 & 3105 & 4781 & 2940 & 4637 & 2653 & 2414 \\
\hline & 3928 & 6426 & 4747 & 7636 & 4915 & 4996 & 3058 & 4523 & 3001 & 5193 & 2911 & 2659 \\
\hline & 3459 & 6441 & 4544 & 6936 & 5052 & 4923 & 2968 & 4208 & 2966 & 5074 & 2737 & 2675 \\
\hline & 3754 & 5866 & 5041 & 7673 & 4487 & 4512 & 2840 & 4417 & 2703 & 4580 & 2863 & 2641 \\
\hline & 3286 & 6873 & 4615 & 7141 & 4243 & 4069 & 3092 & 4337 & 2614 & 4475 & 2628 & 2430 \\
\hline & 3260 & 5947 & 4358 & 6536 & 4493 & 4774 & 3379 & 4340 & 3173 & 4448 & 2529 & 2659 \\
\hline & 3210 & 5719 & 4999 & 7265 & 4837 & 4839 & 3069 & 4850 & 2898 & 4509 & 2767 & 2276 \\
\hline & 3444 & 6012 & 4908 & 7517 & 4794 & 4219 & 2742 & 4365 & 3076 & 4842 & 2807 & 2647 \\
\hline & 3916 & 6560 & 4620 & 7660 & 5132 & 5140 & 3448 & 4273 & 3137 & 4368 & 2852 & 2410 \\
\hline & 3491 & 6347 & 4405 & 6549 & 5174 & 4703 & 3143 & 4781 & 2704 & 4597 & 2802 & 2285 \\
\hline \multirow{8}{*}{ Thrombin } & 5131 & 6883 & 6602 & 16709 & 12493 & 12386 & 3910 & 7075 & 4745 & 10485 & 6235 & 4373 \\
\hline & 4678 & 6971 & 6974 & 18336 & 10449 & 10751 & 3371 & 8038 & 4662 & 10604 & 6275 & 4787 \\
\hline & 4781 & 6833 & 6991 & 17708 & 11845 & 10128 & 3527 & 7617 & 4494 & 11819 & 6250 & 4374 \\
\hline & 4902 & 7583 & 7533 & 19233 & 11918 & 10528 & 3718 & 7973 & 4285 & 10782 & 6298 & 4505 \\
\hline & 4872 & 7965 & 6890 & 16485 & 12302 & 10332 & 3913 & 6738 & 4451 & 10719 & 6438 & 4904 \\
\hline & 5206 & 7132 & 7506 & 15878 & 12729 & 10875 & 4056 & 7174 & 4603 & 11288 & 6696 & 4881 \\
\hline & 5421 & 6527 & 7438 & 15503 & 11901 & 11028 & 4141 & 7292 & 4826 & 11093 & 6591 & 4847 \\
\hline & 5273 & 7097 & 6849 & 19380 & 11674 & 10921 & 4251 & 7324 & 4564 & 10405 & 6060 & 4629 \\
\hline
\end{tabular}




\begin{tabular}{|c|c|c|c|c|c|c|c|c|c|c|c|c|}
\hline & 5633 & 7292 & 7003 & 16928 & 11725 & 10779 & 4070 & 7899 & 4756 & 10542 & 6048 & 4197 \\
\hline & 5316 & 7627 & 7013 & 16561 & 11145 & 11850 & 3678 & 7866 & 4822 & 10201 & 6710 & 4316 \\
\hline & 4852 & 7132 & 7417 & 16586 & 12077 & 12188 & 3469 & 6852 & 4554 & 10852 & 6315 & 4450 \\
\hline & 5239 & 6626 & 6602 & 16434 & 12846 & 9967 & 3516 & 6867 & 4755 & 11142 & 6817 & 4236 \\
\hline & 4839 & 7193 & 7545 & 16766 & 12767 & 10395 & 3573 & 7262 & 4623 & 11813 & 6462 & 4396 \\
\hline & 5281 & 6916 & 7588 & 16907 & 13046 & 10558 & 3979 & 7237 & 4974 & 10800 & 6291 & 4525 \\
\hline \multirow{14}{*}{ Control } & 5380 & 5566 & 6891 & 15197 & 9172 & 7959 & 3233 & 5163 & 3708 & 7332 & 5456 & 4150 \\
\hline & 5146 & 5978 & 7071 & 14096 & 8556 & 7690 & 3342 & 5518 & 3562 & 8101 & 5137 & 4649 \\
\hline & 4910 & 5902 & 7554 & 15618 & 9280 & 7625 & 3183 & 5982 & 3452 & 7937 & 6095 & 4588 \\
\hline & 5011 & 6025 & 6335 & 13267 & 9030 & 7279 & 3768 & 6099 & 3509 & 8119 & 6078 & 4446 \\
\hline & 5264 & 6654 & 7451 & 15476 & 9902 & 7534 & 3875 & 5139 & 3447 & 7627 & 5684 & 4502 \\
\hline & 5464 & 6198 & 6023 & 15635 & 10216 & 8296 & 3945 & 5194 & 3523 & 7673 & 5337 & 4139 \\
\hline & 5119 & 6928 & 6339 & 14397 & 9373 & 8002 & 3839 & 5508 & 3796 & 7222 & 5443 & 4055 \\
\hline & 5104 & 6097 & 6312 & 16423 & 8350 & 8073 & 3748 & 6063 & 3601 & 7355 & 5681 & 4116 \\
\hline & 5400 & 6317 & 6485 & 12474 & 8281 & 8295 & 3852 & 5788 & 3393 & 7239 & 5107 & 4253 \\
\hline & 5100 & 6385 & 6632 & 15157 & 8347 & 7273 & 3286 & 5122 & 3270 & 7797 & 4837 & 4344 \\
\hline & 5524 & 6395 & 6221 & 12133 & 8829 & 7284 & 3782 & 5837 & 3750 & 7776 & 5542 & 4505 \\
\hline & 5423 & 6550 & 6222 & 13143 & 8838 & 7109 & 3547 & 5941 & 3362 & 7457 & 5476 & 4073 \\
\hline & 5028 & 6487 & 6505 & 13954 & 9044 & 7249 & 3701 & 6026 & 3512 & 7818 & 5321 & 4059 \\
\hline & 5670 & 6145 & 6621 & 12402 & 9691 & 7279 & 3574 & 6469 & 3504 & 7366 & 4973 & 4646 \\
\hline & \multicolumn{12}{|c|}{ FTIC } \\
\hline & $\mathrm{CH} 1$ & $\mathrm{CH} 2$ & $\mathrm{CH} 3$ & $\mathrm{CH} 4$ & CH5 & CH6 & $\mathrm{CH} 1$ & $\mathrm{CH} 2$ & $\mathrm{CH} 3$ & $\mathrm{CH} 4$ & CH5 & CH6 \\
\hline & 302 & 302 & 302 & 302 & 302 & 302 & 365 & 365 & 365 & 365 & 365 & 365 \\
\hline & 450 & 480 & 505 & 535 & 570 & 605 & 450 & 480 & 505 & 535 & 570 & 605 \\
\hline \multirow{14}{*}{$\begin{array}{l}\text { PLA2 from } \\
\text { Naja atra }\end{array}$} & 3624 & 4702 & 7369 & 3877 & 2019 & 3323 & 3585 & 6529 & 6376 & 6586 & 3454 & 3539 \\
\hline & 3656 & 4150 & 7068 & 3501 & 2803 & 3427 & 3166 & 6296 & 6861 & 6878 & 3398 & 3379 \\
\hline & 4189 & 4971 & 7427 & 3940 & 2005 & 3362 & 3315 & 5834 & 6450 & 6431 & 3296 & 3335 \\
\hline & 3941 & 5833 & 7935 & 3732 & 2255 & 3136 & 3545 & 5910 & 6033 & 6133 & 3395 & 3516 \\
\hline & 3552 & 6484 & 7865 & 4642 & 2565 & 3246 & 3547 & 5350 & 6730 & 6810 & 3567 & 3502 \\
\hline & 3788 & 6791 & 7264 & 3640 & 2605 & 3152 & 3514 & 5879 & 6781 & 6069 & 3532 & 3823 \\
\hline & 4283 & 6635 & 7658 & 4265 & 2496 & 3365 & 3288 & 5297 & 6997 & 6917 & 3758 & 3891 \\
\hline & 4023 & 5437 & 7172 & 4034 & 2956 & 3364 & 3115 & 5653 & 7115 & 6218 & 3410 & 3616 \\
\hline & 3869 & 5562 & 6953 & 4074 & 2255 & 3034 & 3153 & 5071 & 6273 & 6539 & 3695 & 3937 \\
\hline & 4076 & 5508 & 7362 & 3842 & 2380 & 3013 & 4056 & 5575 & 6806 & 6114 & 3781 & 3786 \\
\hline & 3733 & 5820 & 7478 & 3540 & 2585 & 3053 & 3579 & 6081 & 7260 & 6761 & 3917 & 4064 \\
\hline & 3983 & 5579 & 7949 & 4566 & 2553 & 3547 & 3988 & 6110 & 6814 & 6293 & 3579 & 3921 \\
\hline & 4459 & 6755 & 7077 & 4608 & 2775 & 3389 & 3380 & 5344 & 7019 & 6470 & 3386 & 3982 \\
\hline & 4062 & 6241 & 7118 & 4195 & 2517 & 3665 & 3854 & 5345 & 6613 & 6169 & 3434 & 3164 \\
\hline \multirow[t]{3}{*}{ Hyaluronidase } & 4465 & 5352 & 5415 & 4064 & 2212 & 4768 & 4030 & 4353 & 5966 & 7618 & 5169 & 3628 \\
\hline & 4197 & 6002 & 5473 & 3815 & 2237 & 4349 & 3346 & 4460 & 5646 & 7176 & 5242 & 3428 \\
\hline & 4299 & 5458 & 6012 & 4212 & 2327 & 4633 & 3888 & 4750 & 5905 & 7227 & 5231 & 3930 \\
\hline
\end{tabular}




\begin{tabular}{|c|c|c|c|c|c|c|c|c|c|c|c|c|}
\hline \multirow{11}{*}{ Hyaluronidase } & 4039 & 5747 & 5789 & 4018 & 2161 & 4333 & 3953 & 4862 & 5689 & 7524 & 4934 & 3716 \\
\hline & 4206 & 6135 & 5690 & 3808 & 2150 & 4038 & 3703 & 4598 & 5768 & 7584 & 5283 & 3649 \\
\hline & 4258 & 5756 & 5480 & 3913 & 2024 & 4291 & 3606 & 4276 & 5896 & 7164 & 5833 & 3763 \\
\hline & 4368 & 5693 & 5514 & 4510 & 2793 & 4675 & 4190 & 4414 & 5848 & 7874 & 5790 & 3989 \\
\hline & 4736 & 5653 & 5238 & 3812 & 2536 & 4556 & 3777 & 4158 & 6074 & 8159 & 5866 & 3909 \\
\hline & 4240 & 5238 & 5517 & 3969 & 2416 & 4824 & 3530 & 4150 & 5407 & 7819 & 5066 & 4265 \\
\hline & 4638 & 5759 & 5604 & 3890 & 2351 & 4101 & 4160 & 4072 & 5842 & 7286 & 5502 & 3803 \\
\hline & 4434 & 5348 & 5655 & 3963 & 2500 & 4166 & 3435 & 3910 & 5786 & 7897 & 5017 & 4246 \\
\hline & 4810 & 5208 & 5972 & 3888 & 2266 & 4725 & 3693 & 4026 & 5522 & 7455 & 5532 & 3676 \\
\hline & 4644 & 5391 & 5361 & 3879 & 2681 & 4617 & 3587 & 3938 & 6088 & 7422 & 5442 & 4041 \\
\hline & 4711 & 5992 & 5474 & 4423 & 2644 & 4540 & 3793 & 4247 & 5861 & 7977 & 4969 & 4157 \\
\hline \multirow{14}{*}{ Cardiotoxin } & 5683 & 7497 & 12568 & 9496 & 5742 & 7009 & 4999 & 7934 & 10780 & 10758 & 8738 & 7463 \\
\hline & 5774 & 6514 & 12176 & 8588 & 5138 & 6099 & 4410 & 9081 & 10636 & 11224 & 8239 & 7933 \\
\hline & 5239 & 6286 & 12212 & 7957 & 5569 & 6920 & 4663 & 8030 & 11724 & 10989 & 8566 & 7422 \\
\hline & 5882 & 8055 & 12600 & 7671 & 6171 & 6171 & 4765 & 6777 & 13352 & 12020 & 8434 & 7229 \\
\hline & 5788 & 8476 & 13841 & 8307 & 6232 & 6703 & 4949 & 6755 & 13337 & 12668 & 8287 & 7529 \\
\hline & 6213 & 8123 & 13895 & 8804 & 6010 & 6717 & 5162 & 7218 & 13270 & 12733 & 8451 & 7498 \\
\hline & 6248 & 7445 & 13592 & 8747 & 6389 & 6855 & 4918 & 8471 & 13773 & 12972 & 8665 & 7874 \\
\hline & 6137 & 6914 & 15260 & 8844 & 5980 & 6553 & 4968 & 9021 & 12783 & 12768 & 8223 & 7352 \\
\hline & 6268 & 6888 & 14905 & 8644 & 6020 & 6650 & 4714 & 8139 & 12789 & 12784 & 8942 & 7936 \\
\hline & 6484 & 7707 & 14233 & 8463 & 6291 & 6495 & 4606 & 8241 & 13581 & 10667 & 8564 & 8067 \\
\hline & 5912 & 8493 & 14997 & 8429 & 5437 & 6445 & 4711 & 8898 & 13846 & 11558 & 8415 & 7297 \\
\hline & 5941 & 7069 & 14139 & 9682 & 5231 & 6444 & 4405 & 8432 & 14315 & 11311 & 8509 & 7732 \\
\hline & 5592 & 7855 & 13650 & 8588 & 5289 & 6824 & 4241 & 8204 & 13818 & 11694 & 8656 & 7835 \\
\hline & 5763 & 7392 & 14308 & 8335 & 5188 & 7139 & 4816 & 8197 & 14276 & 11843 & 8396 & 7847 \\
\hline \multirow{14}{*}{$\alpha$-Cobratoxin } & 6015 & 6256 & 15615 & 12166 & 6412 & 7091 & 4577 & 6961 & 10249 & 13558 & 8196 & 7589 \\
\hline & 5884 & 6210 & 17634 & 12424 & 6107 & 7018 & 4389 & 7352 & 12432 & 11680 & 8532 & 7873 \\
\hline & 6068 & 6072 & 15968 & 11097 & 6343 & 7203 & 4440 & 7573 & 10476 & 12273 & 8214 & 7568 \\
\hline & 5876 & 6987 & 16437 & 11216 & 6263 & 7059 & 4743 & 6717 & 12285 & 12899 & 8310 & 7620 \\
\hline & 5917 & 6526 & 17206 & 10442 & 6922 & 7308 & 4830 & 7131 & 11876 & 12901 & 8298 & 7221 \\
\hline & 6093 & 7635 & 16737 & 10358 & 6918 & 7025 & 4928 & 7930 & 11839 & 13267 & 8433 & 7303 \\
\hline & 5963 & 6868 & 16363 & 9992 & 6804 & 7099 & 4866 & 8117 & 12269 & 13117 & 8452 & 7487 \\
\hline & 6227 & 6688 & 16602 & 10321 & 6616 & 6733 & 4955 & 7025 & 12883 & 12916 & 8213 & 7456 \\
\hline & 6377 & 6077 & 16347 & 10380 & 6836 & 6981 & 5050 & 7883 & 12908 & 13071 & 7985 & 7346 \\
\hline & 6193 & 6000 & 16869 & 10461 & 6413 & 7124 & 5083 & 7527 & 12428 & 12748 & 8045 & 7601 \\
\hline & 6272 & 7465 & 15343 & 11159 & 6170 & 6994 & 4693 & 7818 & 12840 & 13003 & 8605 & 7730 \\
\hline & 6534 & 7252 & 15057 & 13386 & 6943 & 7157 & 4370 & 7513 & 11377 & 11937 & 8798 & 7947 \\
\hline & 6455 & 6988 & 15667 & 12329 & 6921 & 7763 & 4692 & 7246 & 11002 & 12682 & 8834 & 8064 \\
\hline & 6481 & 6073 & 17645 & 14164 & 6175 & 7790 & 4876 & 6980 & 12176 & 12400 & 8797 & 7842 \\
\hline \multirow{3}{*}{ Haemocoagulase } & 5572 & 6392 & 15103 & 8339 & 5589 & 6274 & 4601 & 7360 & 10241 & 11290 & 8588 & 7536 \\
\hline & 5426 & 6050 & 13048 & 9279 & 5436 & 6906 & 4378 & 7485 & 12127 & 11380 & 8838 & 7069 \\
\hline & 5845 & 6351 & 13804 & 9028 & 5669 & 6821 & 4752 & 7472 & 10904 & 11495 & 8052 & 7672 \\
\hline
\end{tabular}




\begin{tabular}{|c|c|c|c|c|c|c|c|c|c|c|c|c|}
\hline & 5979 & 6171 & 13731 & 9283 & 5673 & 6893 & 4974 & 7061 & 10048 & 11676 & 8607 & 7227 \\
\hline & 5973 & 6991 & 14010 & 9610 & 5752 & 6688 & 4829 & 6645 & 10659 & 12164 & 8690 & 7143 \\
\hline & 6014 & 6908 & 13845 & 9557 & 5492 & 6388 & 5071 & 7165 & 10715 & 12447 & 8682 & 7458 \\
\hline & 5879 & 7366 & 12913 & 10222 & 5497 & 6554 & 5239 & 7994 & 11611 & 12745 & 8930 & 7623 \\
\hline & 5877 & 6843 & 14359 & 9959 & 5426 & 6463 & 5292 & 7394 & 11586 & 13143 & 8335 & 7372 \\
\hline & 6139 & 6708 & 14799 & 10810 & 5670 & 6692 & 5096 & 7454 & 11439 & 13176 & 8065 & 7880 \\
\hline & 6568 & 7097 & 15296 & 11775 & 5878 & 6922 & 5096 & 7219 & 11911 & 11987 & 7930 & 7614 \\
\hline & 6681 & 7848 & 14776 & 9981 & 5214 & 6970 & 4710 & 7189 & 12082 & 13077 & 8281 & 7409 \\
\hline & 6584 & 7112 & 18737 & 9721 & 5240 & 6660 & 4423 & 7680 & 12444 & 12583 & 7849 & 7050 \\
\hline & 6477 & 6197 & 14496 & 8973 & 5521 & 6226 & 4461 & 7678 & 12833 & 11757 & 7944 & 7135 \\
\hline & 5994 & 7725 & 18785 & 11719 & 5693 & 6648 & 5145 & 7321 & 12635 & 11672 & 8776 & 7181 \\
\hline \multirow{14}{*}{ Thrombin } & 6337 & 6138 & 12520 & 9359 & 4996 & 6316 & 4529 & 6133 & 10227 & 10320 & 8554 & 8008 \\
\hline & 6050 & 6133 & 12349 & 9866 & 4943 & 5997 & 4440 & 5185 & 13117 & 10819 & 8814 & 8184 \\
\hline & 6231 & 6649 & 12439 & 8888 & 5135 & 6299 & 4649 & 6182 & 12841 & 10199 & 8552 & 8795 \\
\hline & 6177 & 6838 & 12609 & 8902 & 5070 & 6308 & 4968 & 5857 & 10929 & 11018 & 7997 & 7916 \\
\hline & 5749 & 7052 & 12472 & 8512 & 4961 & 6200 & 5231 & 5328 & 12144 & 11171 & 8077 & 8120 \\
\hline & 6906 & 6756 & 12123 & 8681 & 5198 & 6028 & 5303 & 5327 & 10780 & 11290 & 8160 & 8621 \\
\hline & 6136 & 6370 & 12144 & 8577 & 5561 & 6575 & 5463 & 5854 & 10234 & 11841 & 8765 & 8566 \\
\hline & 5607 & 6787 & 13993 & 9528 & 5578 & 6131 & 5274 & 6326 & 10986 & 12749 & 8169 & 8079 \\
\hline & 5880 & 6363 & 14214 & 9246 & 5728 & 6527 & 5392 & 5576 & 10907 & 11297 & 8026 & 8422 \\
\hline & 6481 & 6529 & 14294 & 10970 & 5710 & 6612 & 5269 & 5725 & 11655 & 10592 & 7996 & 8130 \\
\hline & 6703 & 6611 & 15958 & 10599 & 5135 & 6934 & 5130 & 5545 & 11569 & 12873 & 8376 & 8420 \\
\hline & 6434 & 6797 & 16198 & 9625 & 5633 & 6554 & 4890 & 5837 & 11506 & 11121 & 8547 & 8034 \\
\hline & 6308 & 7018 & 12679 & 10114 & 5865 & 6267 & 4827 & 5141 & 10467 & 10828 & 8587 & 8793 \\
\hline & 6106 & 6760 & 14360 & 9477 & 5591 & 6598 & 5231 & 5076 & 10718 & 10448 & 8930 & 8007 \\
\hline \multirow{14}{*}{ Control } & 5521 & 5406 & 8598 & 6866 & 3312 & 4948 & 3672 & 7464 & 9241 & 10682 & 6649 & 5915 \\
\hline & 5494 & 5598 & 9565 & 6175 & 3478 & 4779 & 3885 & 7501 & 10154 & 10892 & 7162 & 5159 \\
\hline & 5431 & 5925 & 10255 & 6526 & 3487 & 4591 & 4208 & 7824 & 10934 & 11726 & 8063 & 5927 \\
\hline & 5367 & 6344 & 8920 & 7267 & 3489 & 5827 & 5262 & 6949 & 11082 & 13174 & 7649 & 5098 \\
\hline & 5309 & 6492 & 8769 & 6752 & 5705 & 6208 & 5321 & 7264 & 10738 & 13498 & 7632 & 5225 \\
\hline & 4862 & 6771 & 9991 & 6029 & 4908 & 5911 & 4270 & 7331 & 10855 & 10578 & 7559 & 6275 \\
\hline & 5543 & 7245 & 10865 & 6748 & 5005 & 5271 & 4629 & 7022 & 10766 & 11110 & 8307 & 5742 \\
\hline & 5080 & 6869 & 9325 & 7182 & 5357 & 5504 & 4643 & 7514 & 10954 & 11066 & 8231 & 6188 \\
\hline & 4889 & 7331 & 10842 & 7478 & 4696 & 5595 & 4514 & 7656 & 12121 & 11679 & 8220 & 5717 \\
\hline & 5043 & 7257 & 9225 & 6517 & 4553 & 5191 & 4367 & 6981 & 11240 & 11666 & 8739 & 5214 \\
\hline & 5322 & 7687 & 10581 & 7096 & 3490 & 6168 & 3919 & 7550 & 10223 & 11744 & 8425 & 6030 \\
\hline & 5165 & 7287 & 11653 & 6173 & 4693 & 5747 & 4201 & 8034 & 9866 & 10572 & 8963 & 5708 \\
\hline & 5389 & 7445 & 10297 & 7693 & 5845 & 5611 & 4774 & 7597 & 10836 & 12183 & 8794 & 6126 \\
\hline & 5483 & 7244 & 10410 & 6049 & 3942 & 5664 & 3960 & 7619 & 9778 & 11668 & 7293 & 5931 \\
\hline & \multicolumn{12}{|c|}{ SRB } \\
\hline & CH1 & $\mathrm{CH} 2$ & $\mathrm{CH} 3$ & $\mathrm{CH} 4$ & CH5 & CH6 & CH1 & $\mathrm{CH} 2$ & $\mathrm{CH} 3$ & $\mathrm{CH} 4$ & CH5 & CH6 \\
\hline
\end{tabular}




\begin{tabular}{|c|c|c|c|c|c|c|c|c|c|c|c|c|}
\hline & 302 & 302 & 302 & 302 & 302 & 302 & 365 & 365 & 365 & 365 & 365 & 365 \\
\hline & 450 & 480 & 505 & 535 & 570 & 605 & 450 & 480 & 505 & 535 & 570 & 605 \\
\hline \multirow{14}{*}{$\begin{array}{l}\text { PLA2 from } \\
\text { Naja atra }\end{array}$} & 5985 & 5282 & 11284 & 9136 & 4212 & 6179 & 4965 & 7055 & 11105 & 11856 & 9234 & 10019 \\
\hline & 5935 & 6658 & 11676 & 8967 & 4263 & 6818 & 4808 & 7611 & 10968 & 11916 & 9711 & 9234 \\
\hline & 6244 & 5731 & 11720 & 9491 & 4591 & 7072 & 4962 & 7336 & 10493 & 11330 & 8307 & 8955 \\
\hline & 6416 & 6800 & 12534 & 8847 & 4595 & 6281 & 5157 & 6777 & 11719 & 11972 & 10232 & 9203 \\
\hline & 6659 & 6915 & 12669 & 8628 & 4470 & 6311 & 5374 & 6743 & 12130 & 12004 & 9916 & 8929 \\
\hline & 6775 & 6840 & 12586 & 8923 & 4461 & 6287 & 5362 & 6419 & 11070 & 11939 & 9519 & 9150 \\
\hline & 6077 & 6220 & 11012 & 8545 & 4559 & 6064 & 5370 & 7332 & 10302 & 12228 & 9478 & 8920 \\
\hline & 5959 & 5638 & 12903 & 8478 & 4766 & 6235 & 5443 & 6968 & 10624 & 12938 & 9223 & 9159 \\
\hline & 6370 & 6270 & 13546 & 8640 & 4350 & 5927 & 5537 & 7571 & 12018 & 10620 & 9700 & 9439 \\
\hline & 6434 & 6043 & 13422 & 8531 & 4458 & 6321 & 5346 & 7060 & 10626 & 11597 & 8948 & 9207 \\
\hline & 7011 & 5560 & 13834 & 8559 & 4310 & 6152 & 5401 & 6827 & 11390 & 11735 & 9645 & 9155 \\
\hline & 6832 & 6086 & 14264 & 9552 & 4623 & 6203 & 5211 & 7108 & 10199 & 10941 & 9466 & 9150 \\
\hline & 6913 & 6630 & 13394 & 8258 & 4921 & 6186 & 5232 & 7053 & 10424 & 12703 & 9386 & 10047 \\
\hline & 6745 & 6503 & 13301 & 8841 & 4711 & 7131 & 5484 & 7503 & 11792 & 11383 & 10925 & 10747 \\
\hline \multirow{14}{*}{ Hyaluronidase } & 5396 & 8001 & 4475 & 5892 & 22262 & 20545 & 4728 & 6611 & 4880 & 6315 & 24259 & 26924 \\
\hline & 5917 & 8483 & 4458 & 5555 & 21082 & 17474 & 4741 & 6736 & 5390 & 6132 & 23840 & 26762 \\
\hline & 5716 & 8065 & 4474 & 5254 & 20318 & 19172 & 4727 & 6234 & 5433 & 5550 & 25636 & 27805 \\
\hline & 5599 & 8214 & 4667 & 5966 & 22097 & 18113 & 4772 & 6402 & 4989 & 6000 & 25490 & 28017 \\
\hline & 5404 & 8138 & 4947 & 5660 & 20691 & 18229 & 5050 & 6610 & 5457 & 5572 & 21070 & 27008 \\
\hline & 5603 & 8459 & 5245 & 6463 & 19188 & 17229 & 4965 & 5914 & 5715 & 6821 & 23430 & 24084 \\
\hline & 6068 & 8913 & 5429 & 6708 & 19122 & 18838 & 5405 & 6438 & 5889 & 6461 & 22190 & 26493 \\
\hline & 5775 & 8634 & 5440 & 6421 & 20822 & 18846 & 5102 & 6238 & 5950 & 6561 & 23226 & 23646 \\
\hline & 5710 & 9083 & 4608 & 6310 & 18975 & 17902 & 5489 & 6874 & 5739 & 6093 & 25385 & 26251 \\
\hline & 5886 & 8018 & 4772 & 6244 & 20372 & 19218 & 5225 & 6233 & 5503 & 6923 & 27237 & 29105 \\
\hline & 5763 & 7856 & 5068 & 6165 & 20721 & 18041 & 5411 & 6622 & 5738 & 6878 & 27908 & 29930 \\
\hline & 6042 & 7837 & 4999 & 5994 & 20459 & 19664 & 5273 & 6511 & 5767 & 6769 & 28247 & 30350 \\
\hline & 6029 & 8243 & 4769 & 5828 & 18743 & 19810 & 4788 & 6751 & 5537 & 6660 & 27162 & 29945 \\
\hline & 5826 & 8062 & 4733 & 5726 & 20108 & 18810 & 5363 & 6527 & 5379 & 6700 & 28299 & 28953 \\
\hline \multirow{12}{*}{ Cardiotoxin } & 7237 & 6662 & 4715 & 2210 & 10051 & 19367 & 4684 & 6068 & 3698 & 3329 & 7328 & 10779 \\
\hline & 6644 & 7371 & 4676 & 2537 & 10628 & 15745 & 5459 & 6269 & 3488 & 3263 & 7355 & 10155 \\
\hline & 6222 & 6328 & 4286 & 2628 & 11657 & 15586 & 4354 & 6377 & 3349 & 3054 & 6933 & 10087 \\
\hline & 6247 & 6826 & 4575 & 2581 & 10520 & 15358 & 4136 & 6151 & 3386 & 3384 & 6942 & 11780 \\
\hline & 6366 & 7319 & 4393 & 2559 & 10776 & 15514 & 4758 & 6313 & 3365 & 3025 & 7537 & 10156 \\
\hline & 6343 & 6848 & 4402 & 2599 & 11309 & 17913 & 4690 & 6267 & 3771 & 3138 & 7364 & 10657 \\
\hline & 6291 & 7740 & 4240 & 2428 & 10044 & 16591 & 5089 & 6590 & 3650 & 3003 & 7402 & 10496 \\
\hline & 6605 & 6304 & 4292 & 2312 & 11321 & 15231 & 4908 & 6786 & 3715 & 2970 & 7740 & 11738 \\
\hline & 6562 & 6336 & 4419 & 2633 & 14305 & 16011 & 5289 & 6608 & 3655 & 3180 & 7377 & 11424 \\
\hline & 7428 & 6383 & 4174 & 2854 & 11085 & 18102 & 5094 & 6430 & 3516 & 3011 & 7005 & 11568 \\
\hline & 6443 & 6273 & 4328 & 2568 & 12529 & 19359 & 5034 & 6234 & 3729 & 3457 & 7509 & 11218 \\
\hline & 7100 & 7760 & 4602 & 2908 & 11751 & 18689 & 5519 & 6744 & 3832 & 3085 & 7971 & 12394 \\
\hline
\end{tabular}




\begin{tabular}{|c|c|c|c|c|c|c|c|c|c|c|c|c|}
\hline & 6644 & 7606 & 4751 & 2765 & 14506 & 19024 & 5551 & 7181 & 3967 & 3217 & 7031 & 12212 \\
\hline & 6535 & 6809 & 4674 & 2445 & 11371 & 16386 & 5703 & 7317 & 3574 & 2831 & 7294 & 12009 \\
\hline \multirow{14}{*}{$\alpha$-Cobratoxin } & 6256 & 6883 & 4532 & 3884 & 15476 & 16597 & 5707 & 7807 & 4755 & 3930 & 8268 & 11449 \\
\hline & 6427 & 6671 & 4333 & 3231 & 17259 & 18304 & 5341 & 7999 & 4856 & 3853 & 7993 & 12538 \\
\hline & 5967 & 6592 & 4215 & 3098 & 16021 & 16470 & 5470 & 7212 & 4666 & 3826 & 7457 & 10954 \\
\hline & 6343 & 6976 & 4331 & 3567 & 14813 & 18323 & 5236 & 7974 & 4314 & 3784 & 8252 & 11545 \\
\hline & 6474 & 6895 & 4591 & 3667 & 15040 & 19069 & 5065 & 7183 & 4265 & 3643 & 7606 & 10446 \\
\hline & 6346 & 7053 & 4126 & 3666 & 13792 & 15583 & 5434 & 7103 & 4707 & 3326 & 7531 & 11344 \\
\hline & 6112 & 7669 & 4352 & 3801 & 14324 & 13101 & 4977 & 8714 & 4304 & 3823 & 7892 & 12384 \\
\hline & 7437 & 6597 & 4960 & 3360 & 17134 & 14090 & 5574 & 7713 & 4952 & 3775 & 7413 & 11206 \\
\hline & 6203 & 6977 & 4433 & 3789 & 15678 & 16828 & 5662 & 8295 & 4439 & 3796 & 7101 & 13260 \\
\hline & 6546 & 6320 & 4117 & 3513 & 18006 & 15815 & 5235 & 7597 & 4201 & 3561 & 7008 & 13511 \\
\hline & 6267 & 7717 & 4438 & 3667 & 16003 & 13587 & 5269 & 8115 & 4163 & 3940 & 7654 & 12810 \\
\hline & 6723 & 8400 & 4351 & 3932 & 17301 & 12982 & 5370 & 7892 & 4401 & 3645 & 7835 & 12674 \\
\hline & 6966 & 8461 & 4105 & 3698 & 16043 & 14749 & 5835 & 7112 & 4869 & 3991 & 7471 & 12693 \\
\hline & 6484 & 8526 & 4332 & 3364 & 14844 & 18762 & 6202 & 7148 & 4263 & 3863 & 7234 & 12118 \\
\hline \multirow{14}{*}{ Haemocoagulase } & 5923 & 5171 & 3422 & 3381 & 11695 & 17064 & 5519 & 7100 & 3758 & 3870 & 4619 & 8661 \\
\hline & 6681 & 5656 & 4141 & 3608 & 12485 & 15863 & 6062 & 7478 & 3358 & 3227 & 4285 & 8350 \\
\hline & 6570 & 5043 & 3861 & 3232 & 13144 & 16520 & 5328 & 6918 & 3278 & 3925 & 4181 & 8162 \\
\hline & 6445 & 5788 & 3039 & 3136 & 13494 & 14721 & 4298 & 8086 & 3670 & 3556 & 4519 & 8041 \\
\hline & 7083 & 6259 & 3577 & 3287 & 13156 & 14394 & 4509 & 7888 & 3431 & 3675 & 4329 & 8146 \\
\hline & 6002 & 5673 & 3724 & 3333 & 11962 & 15664 & 4653 & 7926 & 3650 & 3556 & 4634 & 8316 \\
\hline & 6635 & 5664 & 2637 & 3326 & 11148 & 14735 & 5245 & 7611 & 3637 & 3815 & 4504 & 8189 \\
\hline & 6738 & 5478 & 3675 & 3123 & 10730 & 13780 & 5084 & 7170 & 3973 & 3718 & 4152 & 8160 \\
\hline & 6889 & 5580 & 3313 & 3209 & 12628 & 12931 & 5312 & 7812 & 3946 & 3568 & 4029 & 8586 \\
\hline & 6099 & 5166 & 3989 & 3224 & 11783 & 13021 & 5666 & 8076 & 4043 & 3463 & 4580 & 8091 \\
\hline & 6349 & 5048 & 3607 & 3133 & 12618 & 16865 & 5269 & 8091 & 3885 & 3541 & 4710 & 8690 \\
\hline & 6566 & 5486 & 4021 & 3209 & 10949 & 14964 & 5380 & 7174 & 4033 & 3442 & 4287 & 8660 \\
\hline & 6502 & 5641 & 4028 & 3234 & 10837 & 17400 & 5329 & 7052 & 3810 & 3375 & 4250 & 8438 \\
\hline & 7331 & 6041 & 3663 & 3654 & 10115 & 17362 & 5001 & 6726 & 4034 & 3513 & 4338 & 8335 \\
\hline \multirow{12}{*}{ Thrombin } & 6876 & 5339 & 3046 & 2329 & 9585 & 16827 & 5980 & 6755 & 4802 & 3725 & 4683 & 8672 \\
\hline & 6858 & 5607 & 3196 & 2425 & 9543 & 16326 & 6209 & 6566 & 4408 & 3714 & 4130 & 8408 \\
\hline & 7043 & 5097 & 3060 & 2595 & 10004 & 16159 & 5461 & 7354 & 4268 & 3202 & 4148 & 8708 \\
\hline & 6192 & 5352 & 3155 & 2651 & 9998 & 15268 & 5696 & 8300 & 4970 & 3945 & 4321 & 8275 \\
\hline & 6041 & 5797 & 3269 & 2798 & 9565 & 15027 & 5237 & 7322 & 4542 & 3873 & 4452 & 7982 \\
\hline & 6896 & 5923 & 3871 & 2801 & 9027 & 14983 & 5122 & 7354 & 4327 & 3134 & 4039 & 8074 \\
\hline & 6150 & 5960 & 3560 & 2764 & 10947 & 13516 & 5277 & 7686 & 4101 & 3386 & 4203 & 8482 \\
\hline & 6866 & 5918 & 3877 & 2979 & 11253 & 12869 & 5729 & 7635 & 4466 & 3821 & 4078 & 8830 \\
\hline & 6765 & 5556 & 3556 & 3128 & 10604 & 15200 & 6008 & 7888 & 4744 & 3492 & 4764 & 9030 \\
\hline & 6954 & 5362 & 4034 & 3163 & 10803 & 17003 & 6152 & 8265 & 4804 & 3402 & 4407 & 8608 \\
\hline & 6599 & 5001 & 3518 & 3041 & 9709 & 14976 & 6036 & 8354 & 4706 & 3147 & 4045 & 8294 \\
\hline & 7164 & 6053 & 3866 & 2877 & 10396 & 14521 & 5914 & 8375 & 4942 & 3332 & 4185 & 8007 \\
\hline
\end{tabular}




\begin{tabular}{|c|c|c|c|c|c|c|c|c|c|c|c|c|}
\hline & 6540 & 5103 & 3140 & 2909 & 9610 & 16636 & 5779 & 7177 & 4891 & 3272 & 4114 & 8081 \\
\hline & 6605 & 5619 & 3578 & 2795 & 11411 & 16593 & 6660 & 6489 & 4750 & 3250 & 4735 & 8459 \\
\hline \multirow{14}{*}{ Control } & 6655 & 5218 & 3305 & 4176 & 18325 & 14932 & 4414 & 7676 & 3804 & 4404 & 8424 & 16623 \\
\hline & 7002 & 5822 & 3559 & 4644 & 19092 & 16040 & 4228 & 7822 & 3142 & 3840 & 8094 & 14200 \\
\hline & 7435 & 5341 & 3499 & 4250 & 22006 & 15714 & 3899 & 7441 & 3534 & 3833 & 8383 & 14240 \\
\hline & 7462 & 5798 & 3643 & 4114 & 21037 & 13753 & 4067 & 7343 & 3296 & 4217 & 9145 & 15421 \\
\hline & 6987 & 6309 & 3655 & 4361 & 21720 & 16861 & 4995 & 7661 & 3114 & 4192 & 8638 & 15136 \\
\hline & 6645 & 6280 & 3603 & 4462 & 19596 & 17852 & 5331 & 7352 & 3077 & 4080 & 8236 & 12523 \\
\hline & 6394 & 5475 & 3651 & 4426 & 19249 & 15222 & 5809 & 7636 & 3371 & 4197 & 8647 & 13131 \\
\hline & 6537 & 6661 & 3848 & 4450 & 19328 & 19390 & 6078 & 6995 & 3281 & 4154 & 8656 & 13017 \\
\hline & 5812 & 6156 & 3872 & 4237 & 18671 & 16148 & 6042 & 7674 & 3211 & 4103 & 8220 & 13082 \\
\hline & 6152 & 5280 & 3766 & 4578 & 18709 & 15055 & 5888 & 7485 & 3382 & 4131 & 8121 & 16321 \\
\hline & 6385 & 5200 & 3880 & 4680 & 19374 & 18107 & 5758 & 6887 & 3467 & 4247 & 8120 & 13388 \\
\hline & 6607 & 5841 & 3663 & 4907 & 20629 & 19574 & 5843 & 7277 & 3969 & 4294 & 8957 & 13959 \\
\hline & 6710 & 5902 & 3510 & 4211 & 21549 & 14911 & 6005 & 6827 & 3876 & 4251 & 9110 & 13377 \\
\hline & 7191 & 6604 & 3947 & 4210 & 21495 & 14409 & 5989 & 7059 & 3089 & 4095 & 8420 & 14151 \\
\hline & \multicolumn{12}{|c|}{ TY } \\
\hline & $\mathrm{CH} 1$ & $\mathrm{CH} 2$ & $\mathrm{CH} 3$ & $\mathrm{CH} 4$ & CH5 & CH6 & $\mathrm{CH} 1$ & $\mathrm{CH} 2$ & $\mathrm{CH} 3$ & $\mathrm{CH} 4$ & CH5 & CH6 \\
\hline & 302 & 302 & 302 & 302 & 302 & 302 & 365 & 365 & 365 & 365 & 365 & 365 \\
\hline & 450 & 480 & 505 & 535 & 570 & 605 & 450 & 480 & 505 & 535 & 570 & 605 \\
\hline \multirow{14}{*}{$\begin{array}{l}\text { PLA2 from } \\
\text { Naja atra }\end{array}$} & 11821 & 17655 & 9399 & 7867 & 3607 & 4987 & 15030 & 16916 & 8403 & 9915 & 4812 & 4808 \\
\hline & 12290 & 17163 & 9038 & 7995 & 3706 & 5093 & 15313 & 17154 & 8166 & 9477 & 4450 & 4682 \\
\hline & 13095 & 16083 & 9447 & 7936 & 3610 & 4313 & 15437 & 18333 & 8546 & 9565 & 4291 & 4518 \\
\hline & 11080 & 16320 & 9813 & 7646 & 3805 & 4394 & 16075 & 19205 & 8688 & 9428 & 4713 & 4528 \\
\hline & 12354 & 19077 & 9737 & 7759 & 3616 & 4711 & 16574 & 18895 & 8901 & 9746 & 4780 & 4817 \\
\hline & 11041 & 18131 & 9545 & 7639 & 3483 & 4644 & 16620 & 19308 & 8762 & 9698 & 4952 & 4930 \\
\hline & 11686 & 17226 & 9928 & 7541 & 3715 & 4855 & 16947 & 19058 & 8594 & 9718 & 4940 & 4745 \\
\hline & 11614 & 17094 & 9178 & 8056 & 3626 & 4994 & 15611 & 19057 & 8708 & 9966 & 4588 & 4944 \\
\hline & 11868 & 16283 & 9178 & 8111 & 3631 & 4721 & 15034 & 18645 & 8393 & 9252 & 4529 & 4919 \\
\hline & 11932 & 16604 & 9083 & 7740 & 3821 & 5094 & 14582 & 18193 & 8233 & 9528 & 4307 & 4748 \\
\hline & 12629 & 16559 & 9906 & 8172 & 3906 & 5210 & 14376 & 17338 & 8190 & 9433 & 4433 & 4788 \\
\hline & 13061 & 16372 & 9172 & 7296 & 3407 & 4865 & 14616 & 16260 & 8995 & 9898 & 4640 & 4799 \\
\hline & 11820 & 19011 & 9220 & 7496 & 3848 & 5161 & 14067 & 16549 & 8701 & 9873 & 4743 & 5043 \\
\hline & 12080 & 18888 & 9342 & 7408 & 3852 & 4512 & 14537 & 16154 & 8384 & 9848 & 4399 & 4393 \\
\hline \multirow{7}{*}{ Hyaluronidase } & 21155 & 24317 & 13766 & 6355 & 3626 & 5423 & 20595 & 24373 & 12758 & 10187 & 5768 & 4293 \\
\hline & 21812 & 23838 & 14881 & 6762 & 3698 & 5638 & 19916 & 23913 & 11905 & 10277 & 6033 & 4045 \\
\hline & 20785 & 26766 & 12961 & 6959 & 3631 & 5706 & 20781 & 21946 & 11601 & 10093 & 6087 & 4064 \\
\hline & 21150 & 26334 & 14087 & 7138 & 3631 & 5814 & 20391 & 22925 & 12039 & 10389 & 5798 & 4070 \\
\hline & 20285 & 26462 & 13574 & 6186 & 3972 & 5642 & 20271 & 23923 & 12376 & 11002 & 6103 & 4294 \\
\hline & 20727 & 25574 & 13840 & 7129 & 3687 & 5950 & 20793 & 24941 & 13074 & 11498 & 6638 & 4378 \\
\hline & 21440 & 26730 & 14468 & 7287 & 3689 & 6173 & 22376 & 25381 & 13475 & 10939 & 6053 & 4599 \\
\hline
\end{tabular}




\begin{tabular}{|c|c|c|c|c|c|c|c|c|c|c|c|c|}
\hline \multirow[t]{7}{*}{ Hyaluronidase } & 21423 & 26610 & 15283 & 6557 & 3604 & 5877 & 22490 & 25945 & 13828 & 11880 & 5896 & 4507 \\
\hline & 20518 & 26526 & 13391 & 6664 & 3597 & 5933 & 22200 & 27257 & 13669 & 11493 & 6624 & 4471 \\
\hline & 20015 & 29033 & 14116 & 6608 & 3550 & 5660 & 22018 & 26996 & 13103 & 11088 & 6228 & 4416 \\
\hline & 20630 & 29897 & 14783 & 6555 & 3343 & 5902 & 21745 & 27125 & 13243 & 11069 & 6230 & 4321 \\
\hline & 21817 & 29210 & 14212 & 6846 & 3834 & 5580 & 21861 & 27231 & 13486 & 11733 & 6453 & 4467 \\
\hline & 21278 & 29297 & 15704 & 6539 & 3619 & 6224 & 21386 & 25743 & 12846 & 11434 & 6203 & 4401 \\
\hline & 23132 & 29701 & 13278 & 6688 & 3656 & 5777 & 22358 & 26228 & 13719 & 12219 & 6178 & 4684 \\
\hline \multirow{14}{*}{ Cardiotoxin } & 10327 & 13624 & 6876 & 7522 & 3880 & 4174 & 14098 & 15673 & 8036 & 8654 & 3056 & 4269 \\
\hline & 10728 & 15664 & 6983 & 7906 & 3588 & 4394 & 14144 & 18617 & 7861 & 8343 & 3352 & 4478 \\
\hline & 10534 & 16507 & 6606 & 7945 & 3755 & 4392 & 15230 & 19230 & 8123 & 8704 & 3712 & 4020 \\
\hline & 11908 & 17148 & 7861 & 7470 & 4047 & 4124 & 15845 & 17060 & 8362 & 8984 & 3992 & 4301 \\
\hline & 11491 & 18498 & 6941 & 7932 & 3839 & 4410 & 16467 & 15765 & 8374 & 8968 & 3406 & 4593 \\
\hline & 11446 & 18452 & 6587 & 7246 & 3837 & 4131 & 16579 & 17021 & 8396 & 8494 & 3926 & 4520 \\
\hline & 12699 & 14020 & 7020 & 7591 & 3677 & 4151 & 13703 & 17486 & 7445 & 8640 & 3928 & 4616 \\
\hline & 12792 & 18482 & 6398 & 7311 & 3812 & 4339 & 16535 & 18432 & 8272 & 8238 & 3861 & 4633 \\
\hline & 12575 & 18242 & 7365 & 7816 & 4088 & 4575 & 15739 & 18116 & 7924 & 7965 & 3708 & 4428 \\
\hline & 12129 & 17712 & 7843 & 7536 & 3761 & 4799 & 15023 & 18572 & 8082 & 8990 & 3864 & 4269 \\
\hline & 11089 & 18847 & 7162 & 7399 & 3896 & 4479 & 14452 & 18910 & 7917 & 8047 & 3366 & 4442 \\
\hline & 12268 & 16136 & 7444 & 7807 & 3640 & 4424 & 14042 & 17137 & 6796 & 8751 & 3622 & 4361 \\
\hline & 10809 & 14762 & 7642 & 7359 & 3662 & 4645 & 14845 & 17588 & 7828 & 8073 & 3620 & 4146 \\
\hline & 11204 & 13797 & 6388 & 7482 & 3680 & 4477 & 14557 & 17396 & 6742 & 7989 & 3134 & 4244 \\
\hline \multirow{14}{*}{$\alpha$-Cobratoxin } & 10352 & 14031 & 8516 & 8527 & 3476 & 4738 & 14709 & 19157 & 8416 & 9364 & 4249 & 4021 \\
\hline & 10181 & 14888 & 8995 & 9161 & 3228 & 4486 & 15507 & 18276 & 8705 & 9668 & 4135 & 4296 \\
\hline & 10792 & 15049 & 8841 & 9022 & 3295 & 4591 & 15695 & 17975 & 8806 & 9556 & 4523 & 4408 \\
\hline & 10662 & 17152 & 8563 & 9077 & 3195 & 4024 & 16100 & 17190 & 8879 & 9629 & 4751 & 4578 \\
\hline & 10371 & 18051 & 8776 & 9154 & 3390 & 4294 & 16632 & 19998 & 8573 & 10199 & 4122 & 4714 \\
\hline & 11526 & 18433 & 8714 & 8459 & 3330 & 4538 & 16310 & 19500 & 8888 & 9876 & 4160 & 4585 \\
\hline & 10981 & 16608 & 8867 & 8989 & 2964 & 4081 & 15027 & 19313 & 8468 & 9676 & 4491 & 4764 \\
\hline & 10998 & 16550 & 8069 & 8292 & 3405 & 4410 & 16555 & 19746 & 8622 & 9332 & 4221 & 4474 \\
\hline & 10024 & 15130 & 8699 & 8369 & 3426 & 4556 & 14898 & 19049 & 8388 & 9046 & 4209 & 4382 \\
\hline & 12069 & 15714 & 8618 & 8635 & 3684 & 4751 & 13517 & 18828 & 8373 & 10060 & 4152 & 4234 \\
\hline & 12331 & 16732 & 8929 & 8734 & 3246 & 4837 & 12946 & 18838 & 8933 & 9356 & 4274 & 4391 \\
\hline & 11733 & 16661 & 8846 & 8726 & 3325 & 4594 & 13261 & 17990 & 8276 & 9216 & 4431 & 4289 \\
\hline & 10317 & 17526 & 8237 & 9151 & 3217 & 4022 & 13037 & 17922 & 8325 & 10011 & 4611 & 4660 \\
\hline & 10070 & 16552 & 8515 & 8045 & 3377 & 4096 & 13322 & 16757 & 8949 & 9574 & 4232 & 4553 \\
\hline \multirow{7}{*}{ Haemocoagulase } & 14108 & 15684 & 8890 & 9589 & 3027 & 4386 & 12805 & 15992 & 8049 & 9140 & 3744 & 4490 \\
\hline & 12132 & 15844 & 8966 & 9717 & 3194 & 4079 & 12881 & 17628 & 7844 & 9312 & 3865 & 4255 \\
\hline & 11863 & 16809 & 8835 & 8945 & 3367 & 4373 & 13542 & 18575 & 8253 & 9587 & 3942 & 4310 \\
\hline & 12782 & 18511 & 9071 & 9757 & 3657 & 4343 & 14124 & 19890 & 8598 & 10345 & 3693 & 4771 \\
\hline & 13168 & 18690 & 8560 & 9113 & 3701 & 4373 & 14364 & 17006 & 8741 & 10130 & 3968 & 4789 \\
\hline & 14105 & 18918 & 8405 & 9180 & 3301 & 4213 & 13772 & 16045 & 8771 & 9969 & 4012 & 4632 \\
\hline & 12931 & 18040 & 8059 & 9397 & 3226 & 4456 & 13464 & 18784 & 8558 & 9397 & 4005 & 4523 \\
\hline
\end{tabular}




\begin{tabular}{|c|c|c|c|c|c|c|c|c|c|c|c|c|}
\hline \multirow[t]{7}{*}{ Haemocoagulase } & 12811 & 17097 & 8742 & 9389 & 3448 & 4367 & 12970 & 15794 & 8265 & 9419 & 3897 & 4432 \\
\hline & 13477 & 17123 & 8890 & 9046 & 3686 & 4634 & 11921 & 19940 & 7956 & 9096 & 3870 & 4475 \\
\hline & 13281 & 16914 & 8147 & 9296 & 3030 & 4723 & 11605 & 19661 & 7888 & 9009 & 3867 & 4404 \\
\hline & 13241 & 16651 & 8665 & 9519 & 3459 & 4960 & 11495 & 18778 & 8014 & 9509 & 3944 & 4490 \\
\hline & 13309 & 16919 & 8705 & 9790 & 3389 & 4690 & 11346 & 18577 & 8324 & 9478 & 3841 & 4412 \\
\hline & 13279 & 17650 & 8266 & 9071 & 3843 & 4817 & 12227 & 18699 & 8430 & 10110 & 3845 & 4605 \\
\hline & 13808 & 17682 & 8301 & 9675 & 3425 & 4166 & 11996 & 16469 & 8257 & 8878 & 3663 & 4005 \\
\hline \multirow{14}{*}{ Thrombin } & 10295 & 12571 & 6431 & 8985 & 3160 & 4090 & 11528 & 14808 & 6799 & 8411 & 4428 & 4264 \\
\hline & 9273 & 12900 & 6647 & 8356 & 3292 & 4148 & 11303 & 13034 & 6982 & 9042 & 4236 & 4094 \\
\hline & 9371 & 12297 & 6782 & 8488 & 3367 & 4254 & 11524 & 13509 & 6193 & 8995 & 4742 & 4064 \\
\hline & 9948 & 12305 & 6574 & 7823 & 3265 & 4496 & 11169 & 13965 & 6848 & 9074 & 4176 & 4346 \\
\hline & 9894 & 12496 & 6503 & 8770 & 3885 & 4311 & 11434 & 14006 & 7151 & 9656 & 4768 & 4565 \\
\hline & 9873 & 14264 & 6312 & 8914 & 3825 & 4202 & 10994 & 13908 & 6595 & 10190 & 4866 & 5010 \\
\hline & 10438 & 15544 & 6304 & 8234 & 3602 & 4299 & 11657 & 14664 & 6968 & 9461 & 4631 & 5066 \\
\hline & 9413 & 14553 & 6824 & 8942 & 3532 & 4133 & 11790 & 14850 & 6870 & 9650 & 4468 & 4963 \\
\hline & 9685 & 17023 & 6751 & 8033 & 3679 & 4236 & 11480 & 15188 & 6595 & 9393 & 4293 & 4842 \\
\hline & 10858 & 14012 & 7028 & 9094 & 3065 & 4301 & 10457 & 13831 & 6699 & 8948 & 4042 & 4666 \\
\hline & 9276 & 12342 & 7030 & 7905 & 3600 & 4594 & 11155 & 13599 & 6809 & 8956 & 4272 & 4664 \\
\hline & 9680 & 14742 & 6335 & 8368 & 3067 & 4012 & 9425 & 13780 & 5970 & 9042 & 4604 & 4265 \\
\hline & 9128 & 13116 & 6218 & 8251 & 3058 & 4623 & 9761 & 13831 & 5849 & 8902 & 4175 & 4290 \\
\hline & 9405 & 14541 & 6033 & 8127 & 3083 & 4132 & 9984 & 12530 & 6674 & 8336 & 4102 & 4644 \\
\hline \multirow{14}{*}{ Control } & 10290 & 13771 & 8995 & 7311 & 3796 & 4995 & 16024 & 23112 & 8106 & 10856 & 4163 & 4868 \\
\hline & 11228 & 13722 & 8894 & 7874 & 3279 & 5113 & 15984 & 21570 & 8084 & 10975 & 4782 & 4861 \\
\hline & 12077 & 14130 & 8642 & 7290 & 3419 & 4736 & 15148 & 23638 & 8211 & 10258 & 4860 & 4544 \\
\hline & 12752 & 14690 & 8808 & 8016 & 3121 & 4867 & 15461 & 25188 & 8290 & 10262 & 4436 & 4747 \\
\hline & 10850 & 15241 & 8864 & 7964 & 3699 & 4837 & 14923 & 23778 & 8404 & 10790 & 4757 & 5047 \\
\hline & 10530 & 15481 & 9377 & 8097 & 3086 & 5021 & 17592 & 21645 & 8231 & 11294 & 4743 & 4844 \\
\hline & 12952 & 14138 & 9225 & 7956 & 3109 & 5117 & 15022 & 21919 & 9045 & 10873 & 4575 & 5071 \\
\hline & 13823 & 14807 & 9009 & 8190 & 3250 & 4923 & 17887 & 24262 & 8401 & 10376 & 4585 & 4961 \\
\hline & 10357 & 14506 & 8902 & 7183 & 3356 & 5030 & 14716 & 24566 & 8878 & 10505 & 4404 & 4806 \\
\hline & 10854 & 14433 & 8670 & 7719 & 3784 & 5052 & 14806 & 22665 & 8179 & 9601 & 4439 & 4777 \\
\hline & 10641 & 13520 & 8956 & 7512 & 3894 & 5151 & 16666 & 22799 & 8435 & 9976 & 4505 & 4911 \\
\hline & 10945 & 13053 & 8344 & 7413 & 3742 & 4781 & 15005 & 21258 & 8267 & 9688 & 4536 & 4818 \\
\hline & 10504 & 13127 & 8470 & 7704 & 3999 & 4856 & 17044 & 23395 & 8393 & 10413 & 4833 & 5044 \\
\hline & 11647 & 13596 & 9307 & 8120 & 3891 & 5030 & 15733 & 24554 & 9037 & 10691 & 4979 & 5068 \\
\hline
\end{tabular}



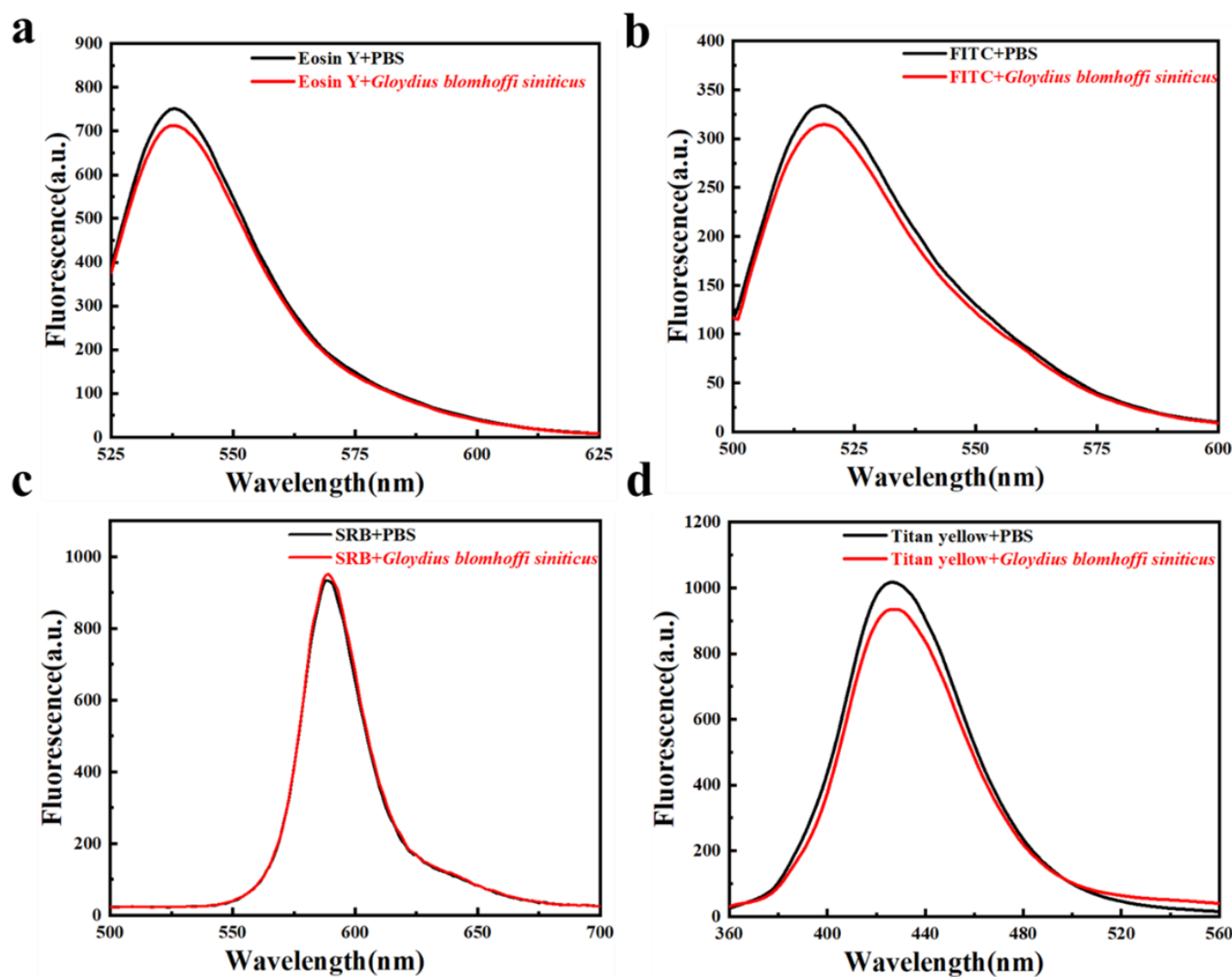

Figure S17 Fluorescence responses of various dyes to venom of Gloydius blomhoffi siniticus. 

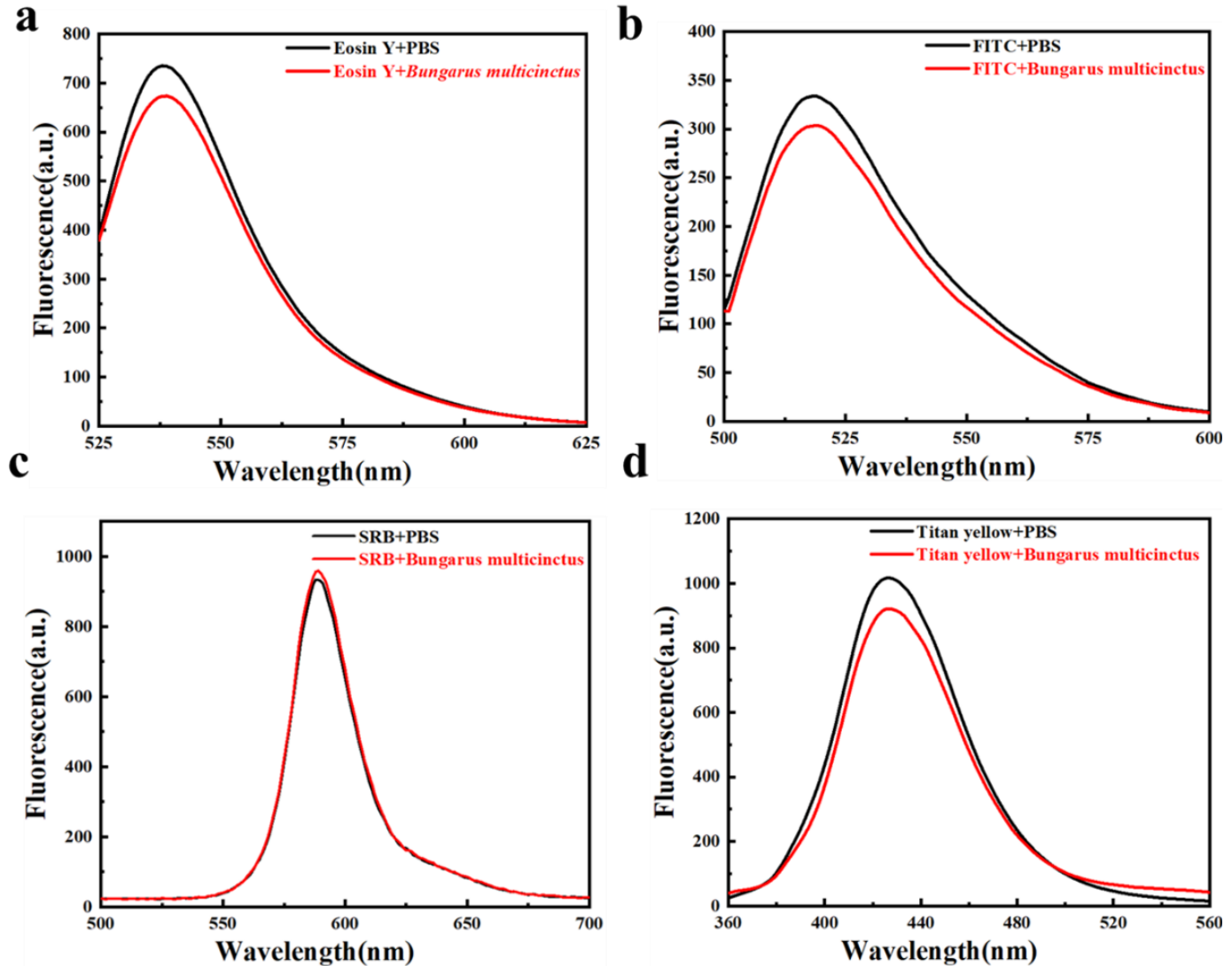

Figure S18 Fluorescence responses of various dyes to venom of Bungarus multicinctus. 

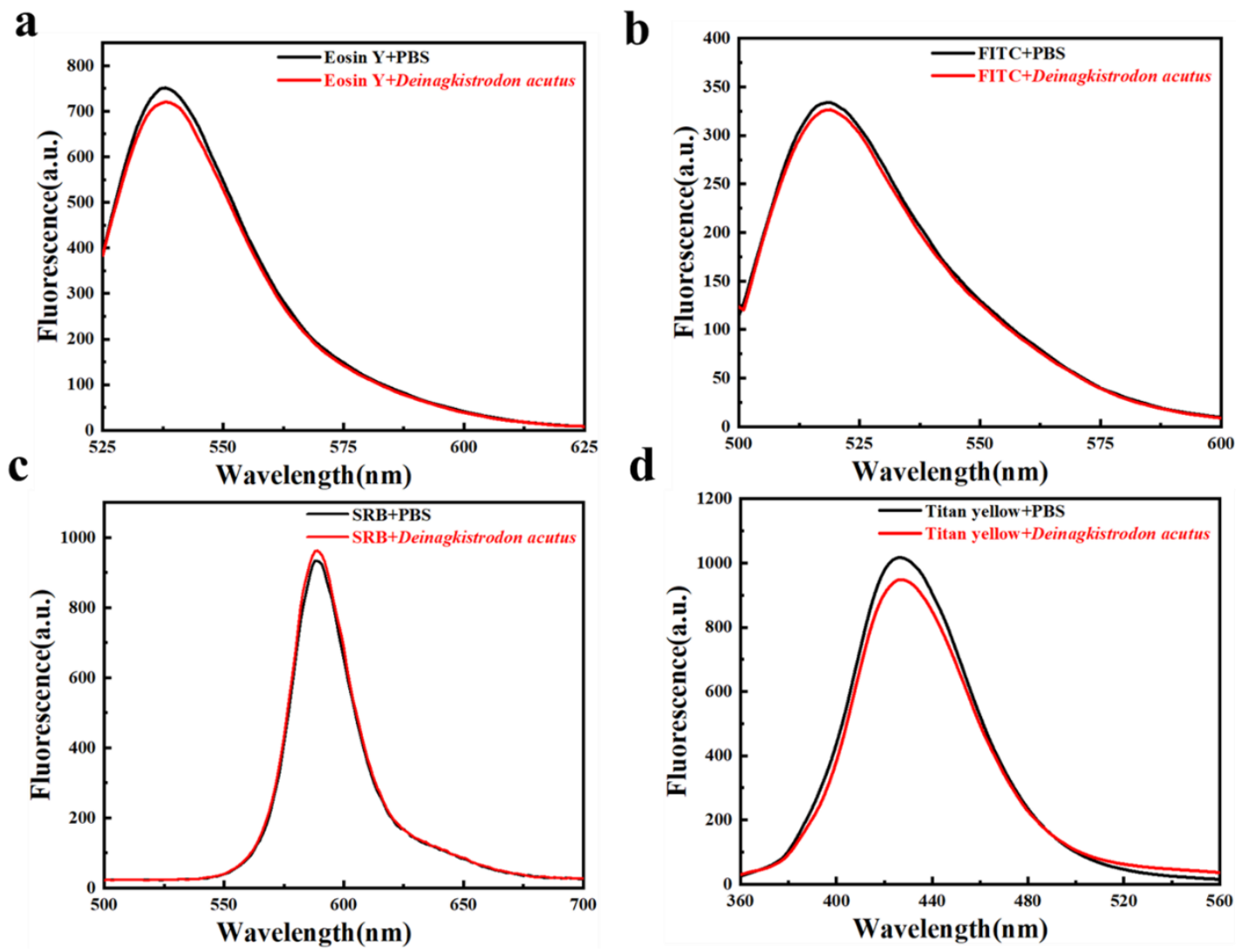

Figure S19 Fluorescence responses of various dyes to venom of Deinagkistrodon acutus. 
a

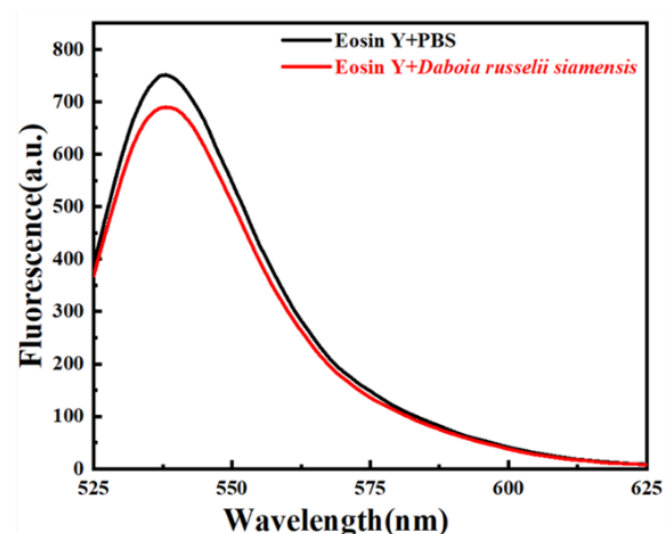

c

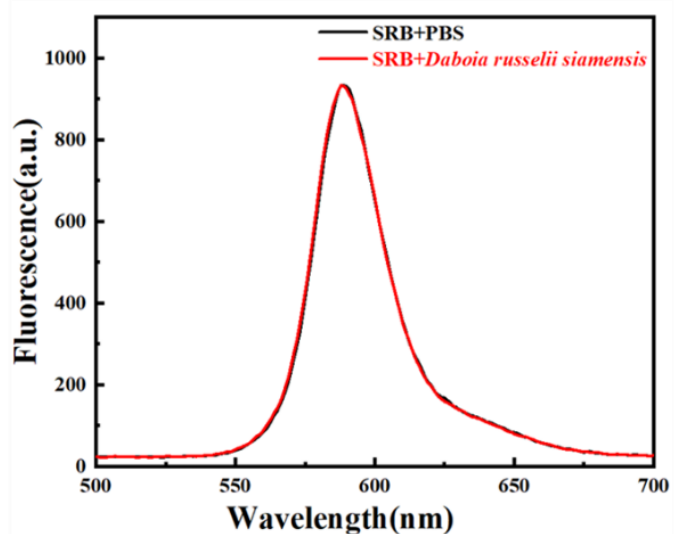

b

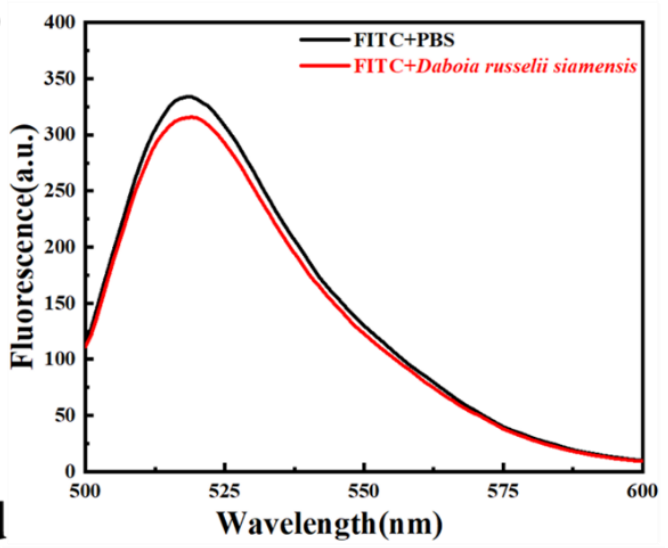

d

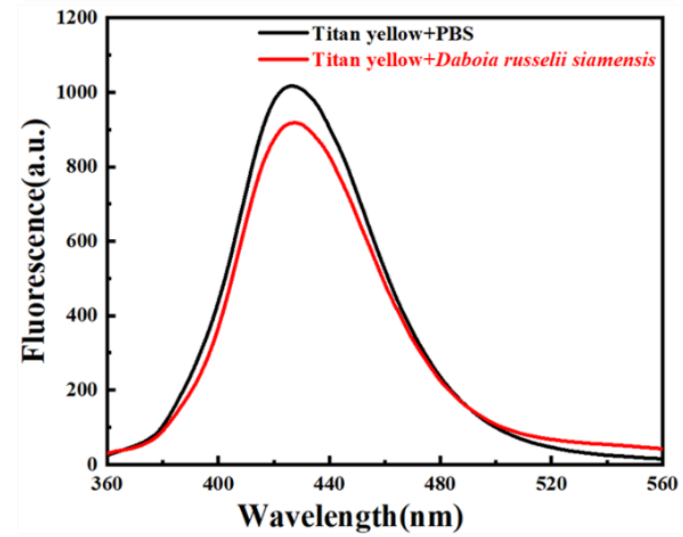

Figure S20 Fluorescence responses of various dyes to venom of Daboia russelii siamensis. 
a

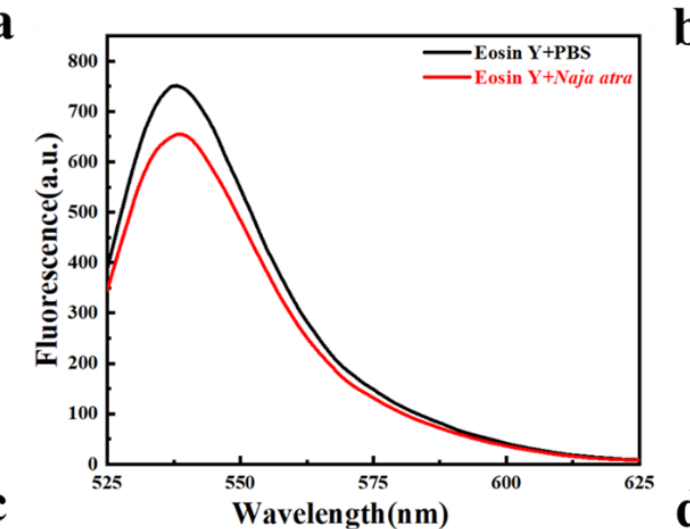

66

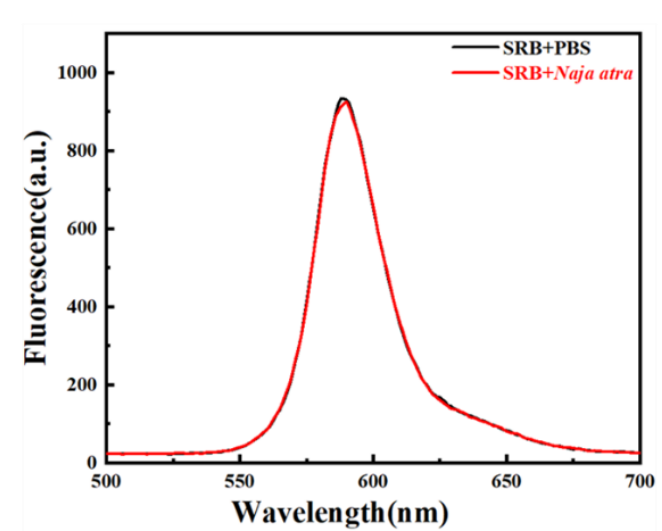

Figure S21 Fluorescence responses of various dyes to venom of Naja atra.
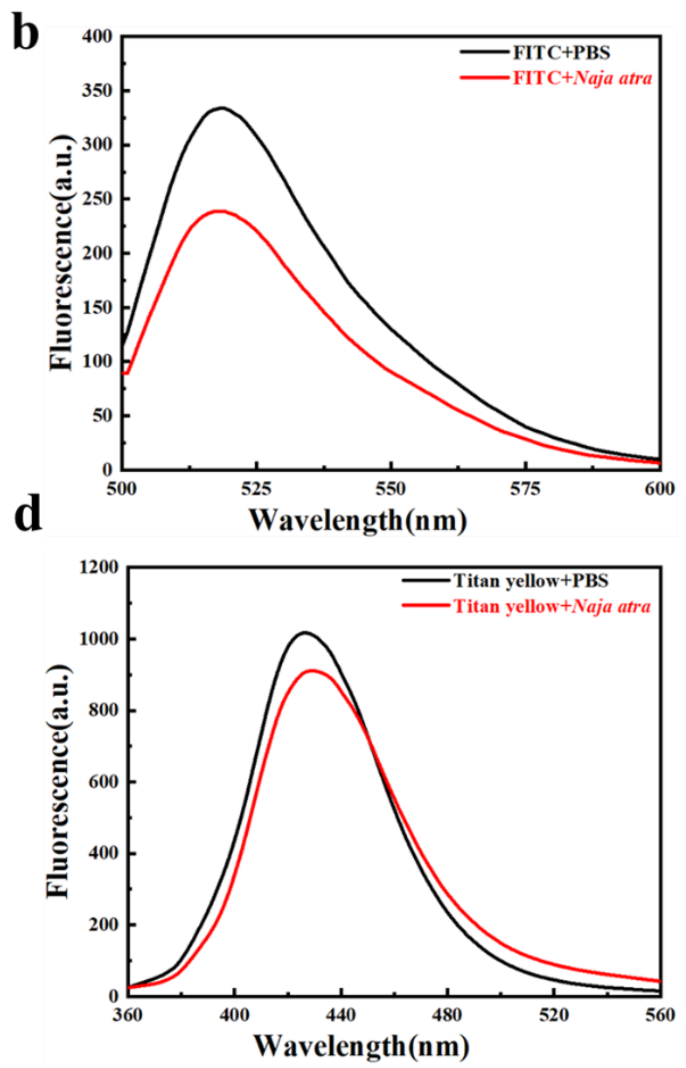
a

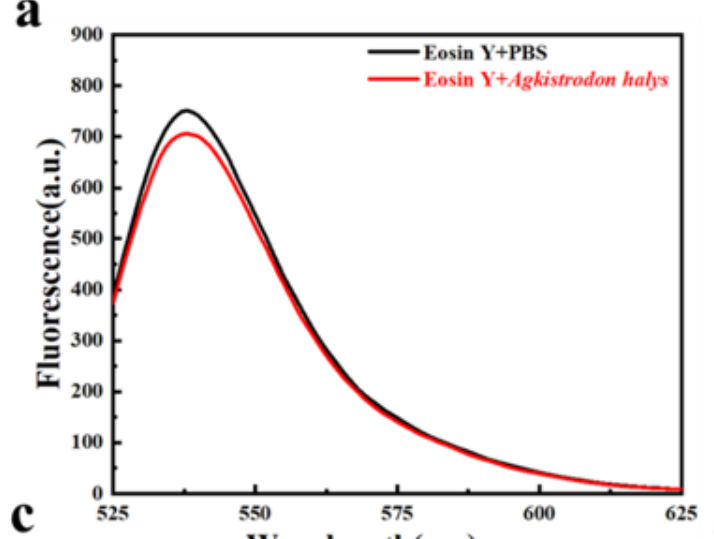

C

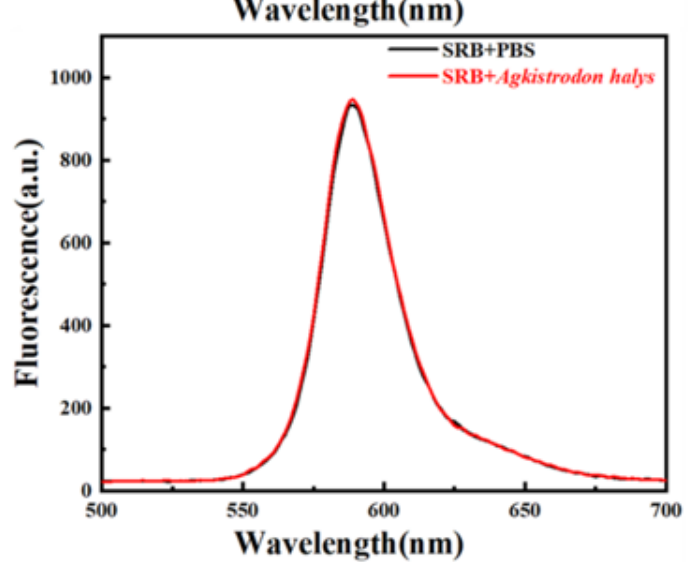

b
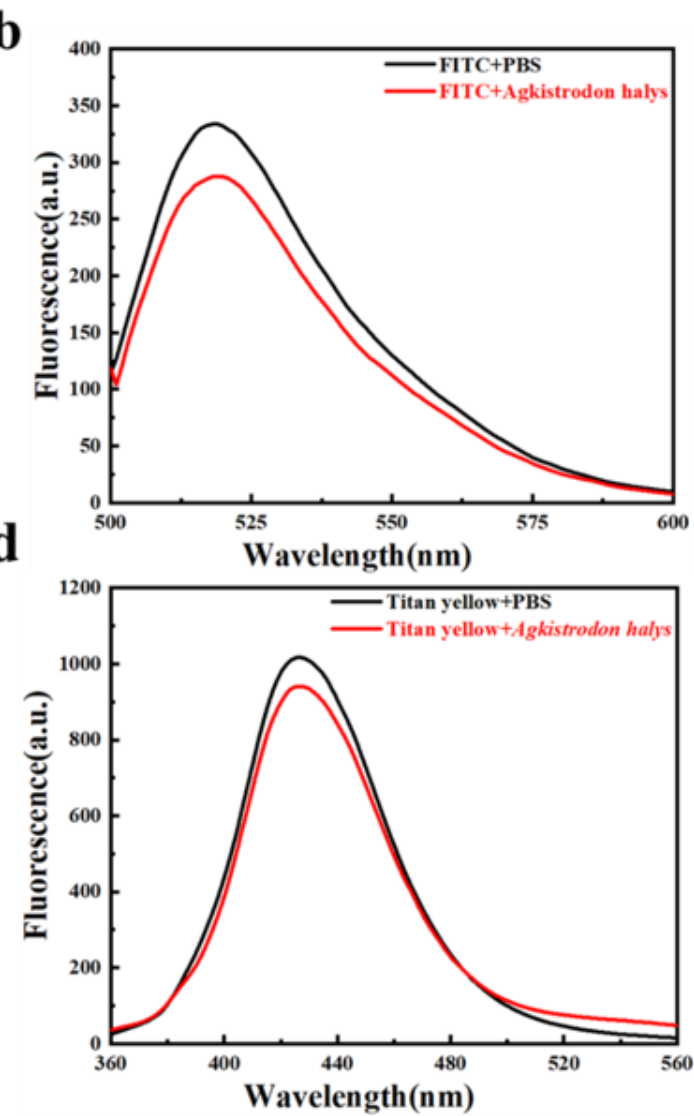

Figure S22 Fluorescence responses of various dyes to venom of Agkistrodon halys. 
a

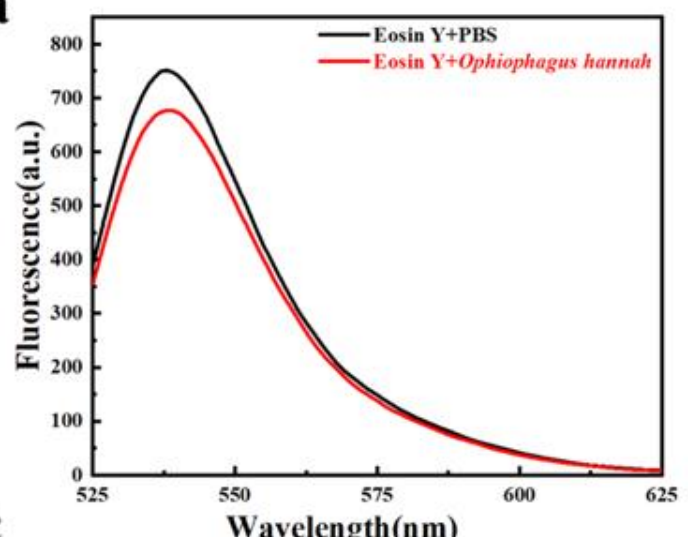

c

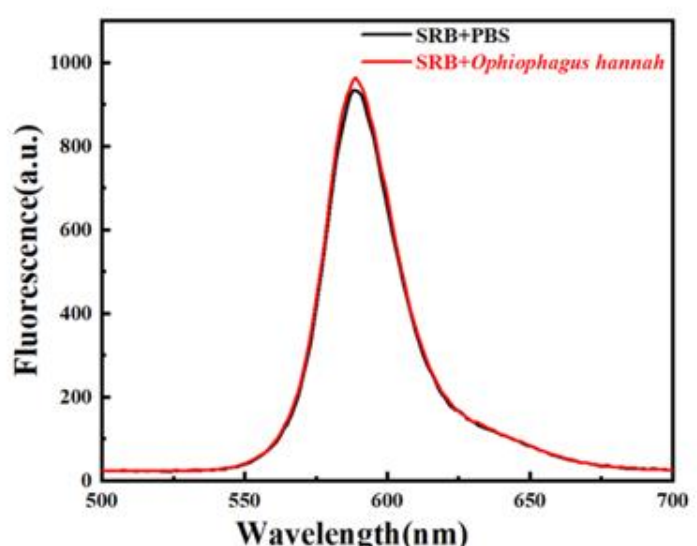

b
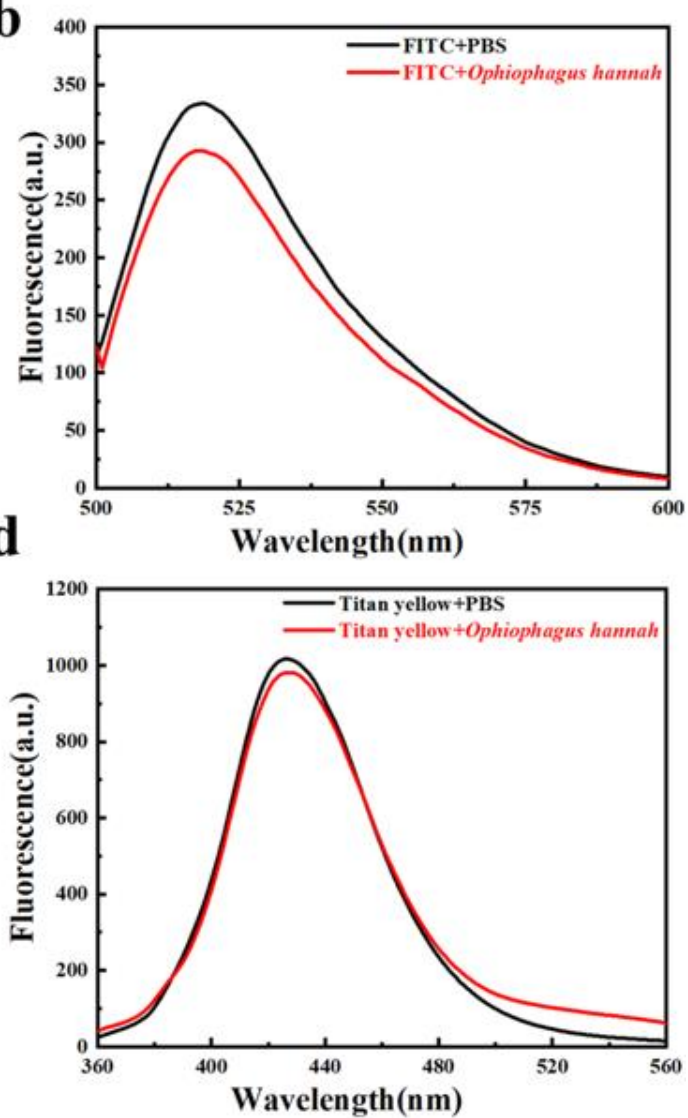

Figure S23 Fluorescence responses of various dyes to venom of Ophiophagus Hannah. 

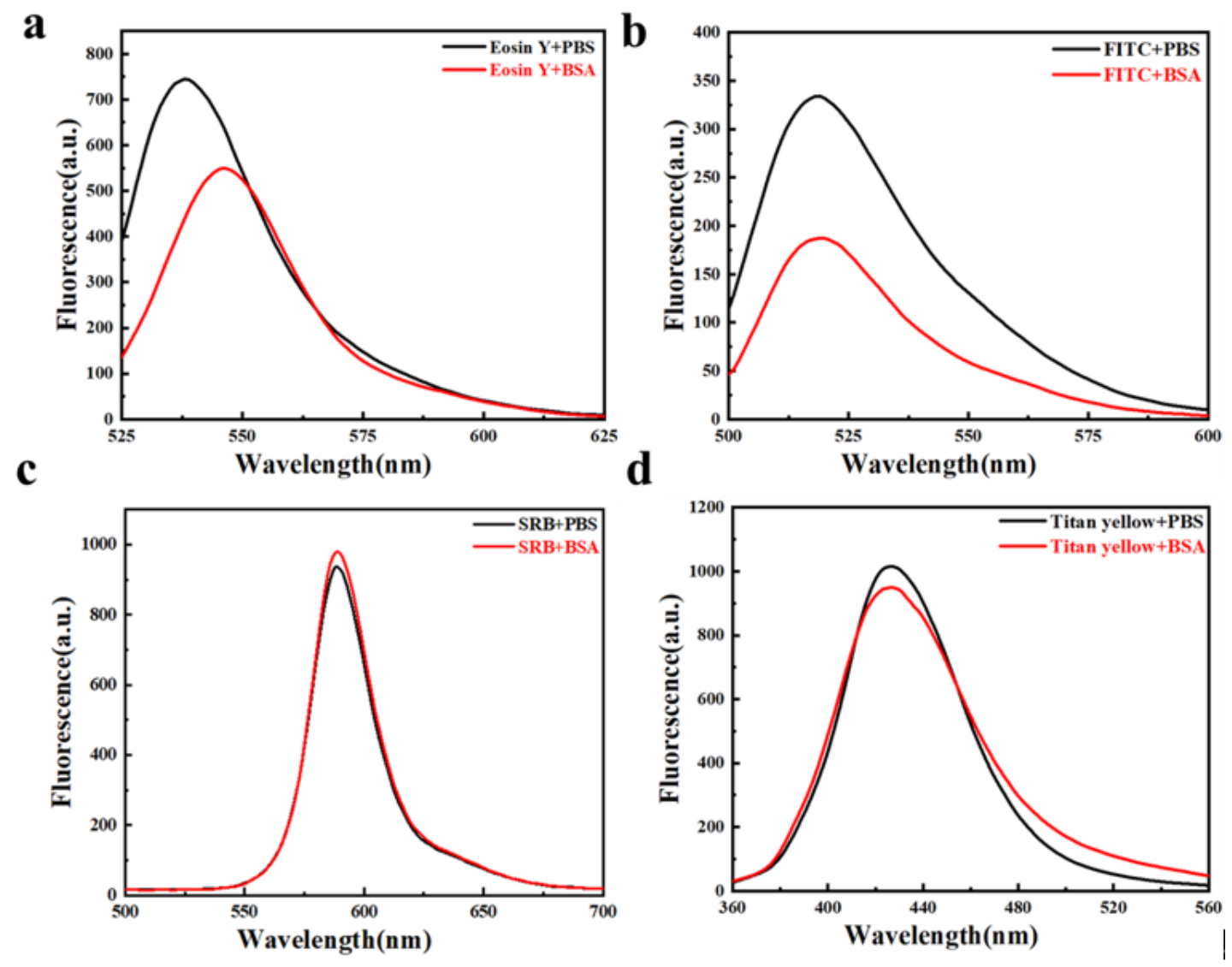

Figure S24 Fluorescence responses of various dyes to BSA. 

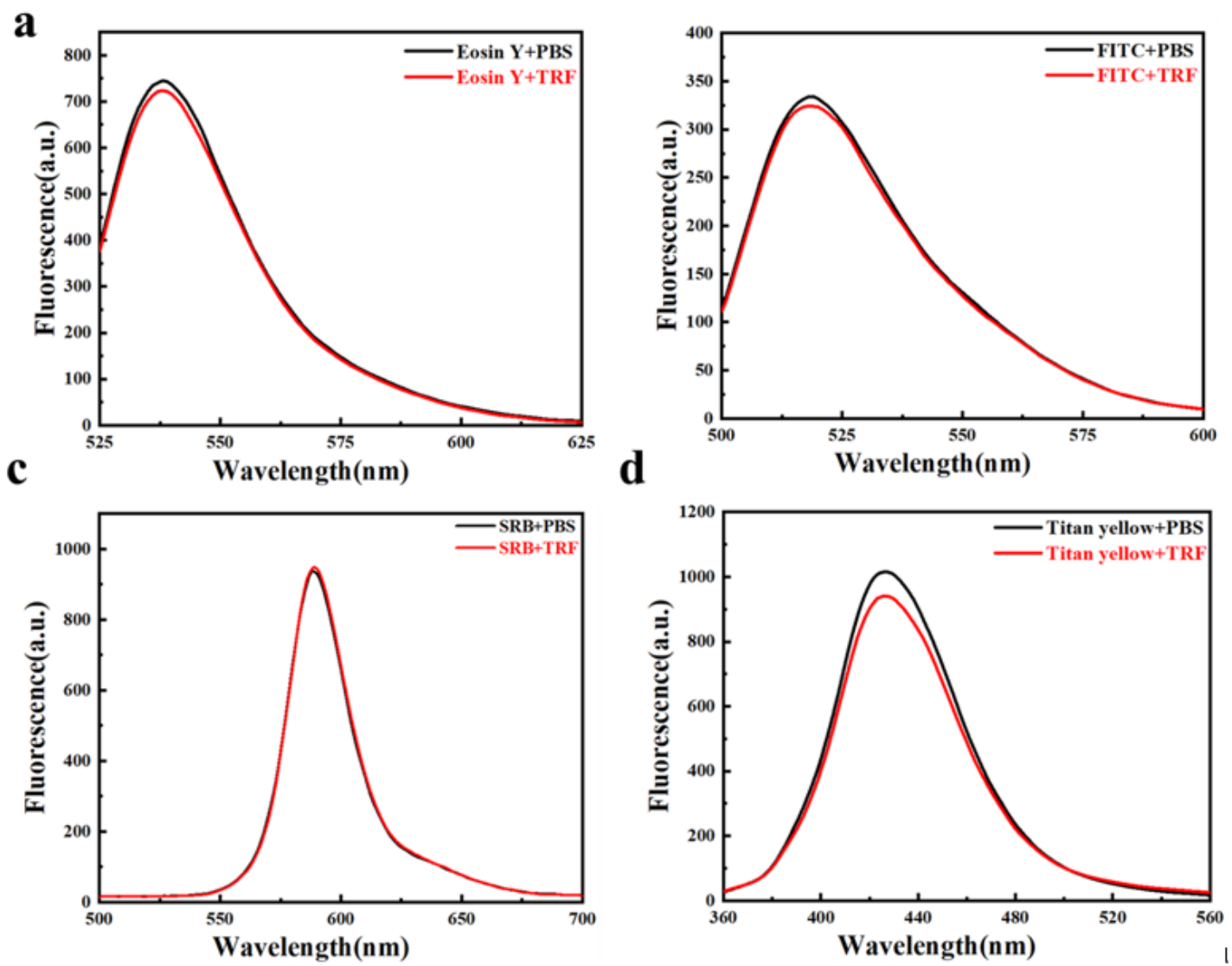

Figure S25 Fluorescence responses of various dyes to TRF. 


\begin{tabular}{|l|r|r|r|r|r|r|r|r|r|}
\hline & Jackknifed Classification Matrix \\
\hline A. halys & A. halys & B. multicinctus & Control & D. acutus & $\begin{array}{c}\text { D. russelii } \\
\text { siamensis }\end{array}$ & $\begin{array}{c}\text { G. blomhoffi } \\
\text { siniticus }\end{array}$ & N. atra & O. hannah & \%correct \\
\hline B. multicinctus & 14 & 0 & 0 & 0 & 0 & 0 & 0 & 0 & 100 \\
\hline Control & 0 & 14 & 0 & 0 & 0 & 0 & 0 & 0 & 100 \\
\hline D. acutus & 0 & 0 & 14 & 0 & 0 & 0 & 0 & 0 & 100 \\
\hline D. russelii siamensis & 0 & 0 & 0 & 14 & 0 & 0 & 0 & 0 & 100 \\
\hline G. blomhoffi siniticus & 0 & 0 & 0 & 0 & 14 & 0 & 0 & 0 & 100 \\
\hline N. atra & 0 & 0 & 0 & 0 & 0 & 14 & 0 & 0 & 100 \\
\hline O. hannah & 0 & 0 & 0 & 0 & 0 & 0 & 14 & 0 & 100 \\
\hline Total & 14 & 0 & 0 & 0 & 0 & 0 & 0 & 14 & 100 \\
\hline
\end{tabular}

b

Canonical Scores Plot

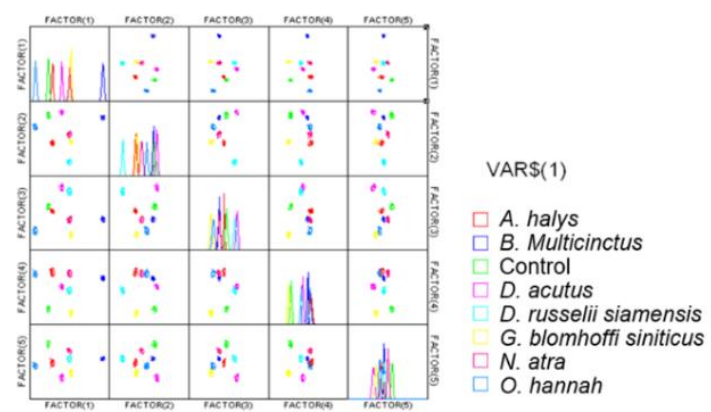

Figure S26 (a) Jackknifed classification matrix of the fluorescent sensor array for the discrimination of different snake venoms in PBS buffer. (b) Canonical scores plot of the fluorescent sensor array for the discrimination of different snake venoms in PBS buffer. 
a

\begin{tabular}{|c|c|c|c|c|c|c|c|c|c|c|c|c|}
\hline & A. halys & B. multicinctus & BSA & Control & D. acutus & $\begin{array}{l}\text { D. russelii } \\
\text { siamensis }\end{array}$ & $\begin{array}{l}\text { G. blomhoffi } \\
\text { siniticus }\end{array}$ & N. atra & O. hannah & TRF & Thrombin & \%correct \\
\hline A. halys & 14 & 0 & 0 & 0 & 0 & 0 & 0 & 0 & 0 & 0 & 0 & 100 \\
\hline B. multicinctus & 0 & 14 & 0 & 0 & 0 & 0 & 0 & 0 & 0 & 0 & 0 & 100 \\
\hline BSA & 0 & 0 & 14 & 0 & 0 & 0 & 0 & 0 & 0 & 0 & 0 & 100 \\
\hline Control & 0 & 0 & 0 & 14 & 0 & 0 & 0 & 0 & 0 & 0 & 0 & 100 \\
\hline D. acutus & 0 & 0 & 0 & 0 & 14 & 0 & 0 & 0 & 0 & 0 & 0 & 100 \\
\hline D. russelii siamensis & 0 & 0 & 0 & 0 & 0 & 14 & 0 & 0 & 0 & 0 & 0 & 100 \\
\hline G. blomhoffi siniticus & 0 & 0 & 0 & 0 & 0 & 0 & 14 & 0 & 0 & 0 & 0 & 100 \\
\hline N. atra & 0 & 0 & 0 & 0 & 0 & 0 & 0 & 14 & 0 & 0 & 0 & 100 \\
\hline O. hannah & 0 & 0 & 0 & 0 & 0 & 0 & 0 & 0 & 14 & 0 & 0 & 100 \\
\hline TRF & 0 & 0 & 0 & 0 & 0 & 0 & 0 & 0 & 0 & 14 & 0 & 100 \\
\hline Thrombin & 0 & 0 & 0 & 0 & 0 & 0 & 0 & 0 & 0 & 0 & 14 & 100 \\
\hline Total & 14 & 14 & 14 & 1 & 14 & 14 & 14 & 14 & 14 & 14 & 14 & 100 \\
\hline
\end{tabular}

Total

Canonical Scores Plot

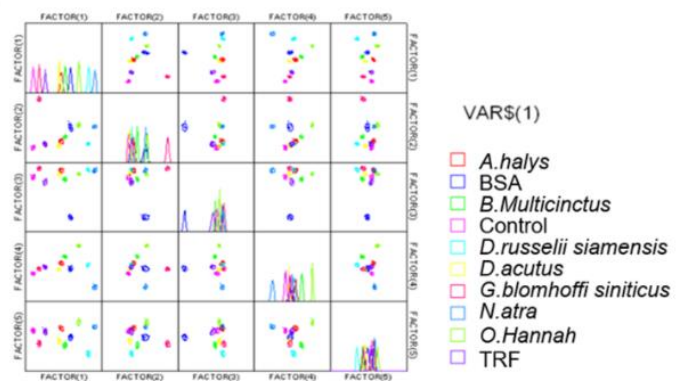

80

Figure S27 (a) Jackknifed classification matrix of the fluorescent sensor array for the discrimination of different

snake venom, BSA, thrombin and TRF in human plasma. (b) Canonical scores plot of the fluorescent sensor array

for the discrimination of different snake venoms, BSA, thrombin and TRF in human plasma. 
Table S2 Raw fluorescence responses in six channels of the fluorescent sensor array in the discrimination of snake

85 venoms in PBS buffer.

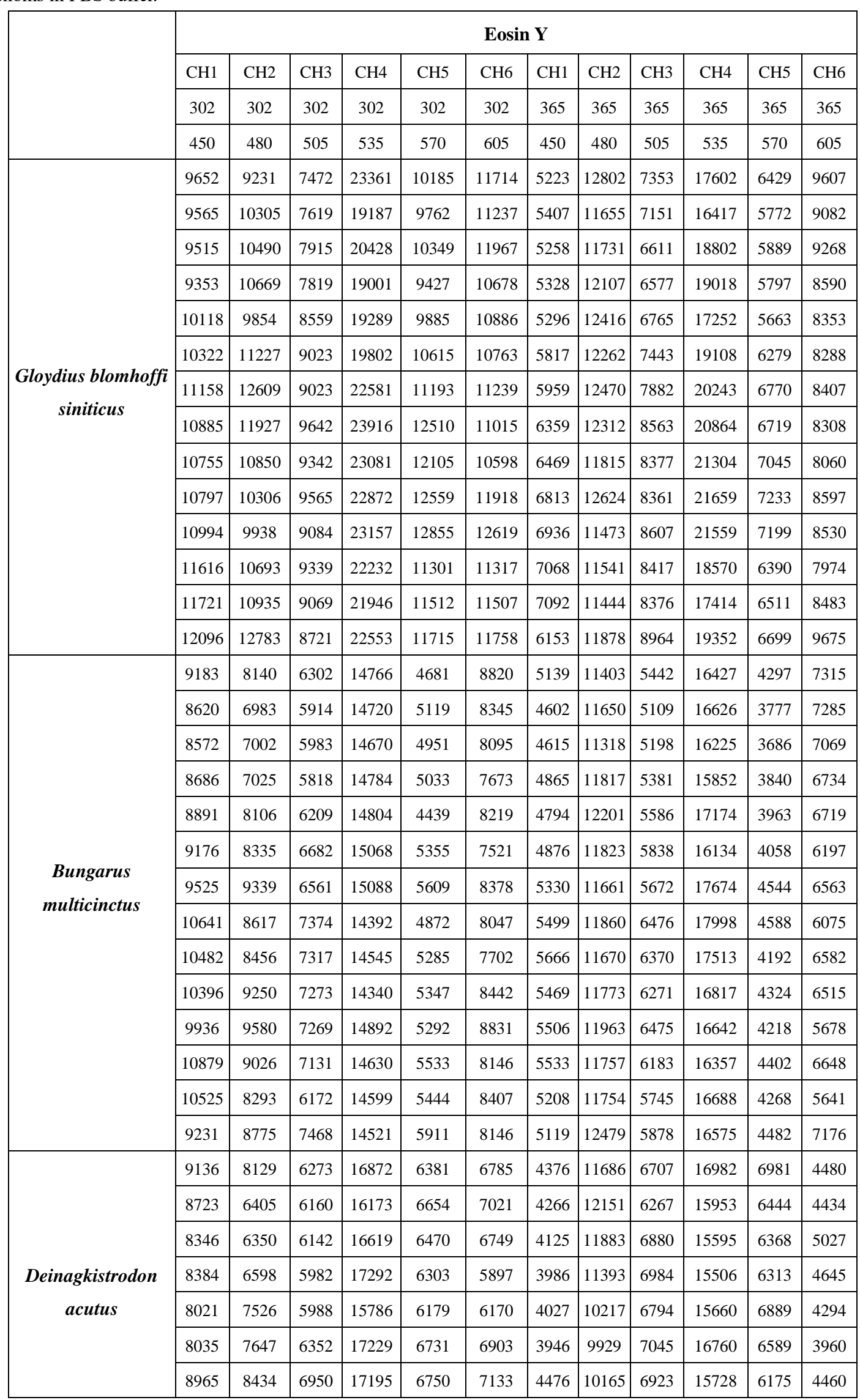




\begin{tabular}{|c|c|c|c|c|c|c|c|c|c|c|c|c|}
\hline & 9962 & 7827 & 7503 & 17252 & 6603 & 7309 & 5281 & 11414 & 6412 & 14908 & 6964 & 4291 \\
\hline & 10749 & 7949 & 7525 & 16646 & 6087 & 6990 & 4646 & 12178 & 7049 & 15041 & 6907 & 4361 \\
\hline & 10160 & 8698 & 7441 & 15664 & 6232 & 6940 & 4575 & 12504 & 6323 & 15040 & 6851 & 4129 \\
\hline & 9887 & 8433 & 6836 & 15798 & 6814 & 6682 & 4890 & 12777 & 6863 & 14835 & 6730 & 4077 \\
\hline & 9657 & 7735 & 7065 & 16234 & 6205 & 7590 & 4846 & 12012 & 6762 & 16522 & 6198 & 4510 \\
\hline & 9203 & 8421 & 7517 & 16889 & 6979 & 6865 & 5028 & 11626 & 6567 & 16650 & 6543 & 4804 \\
\hline & 9780 & 8102 & 7607 & 17186 & 6428 & 6993 & 4717 & 11308 & 6168 & 15242 & 6751 & 5139 \\
\hline & 10190 & 9845 & 8696 & 26020 & 10855 & 7411 & 5837 & 12747 & 7773 & 18918 & 10751 & 7266 \\
\hline & 10069 & 9140 & 9938 & 25924 & 9445 & 6790 & 6107 & 12555 & 8320 & 18360 & 10001 & 7422 \\
\hline & 9892 & 9134 & 8587 & 23799 & 9362 & 6950 & 6129 & 14979 & 7791 & 18935 & 10077 & 7448 \\
\hline & 9816 & 10082 & 8036 & 24824 & 9424 & 6233 & 6193 & 12305 & 7447 & 18836 & 10344 & 6351 \\
\hline & 9511 & 9930 & 7883 & 23185 & 8124 & 5954 & 5891 & 10901 & 7660 & 18653 & 10414 & 6544 \\
\hline & 9777 & 10603 & 8871 & 24397 & 8501 & 5929 & 6031 & 10622 & 9066 & 19369 & 10017 & 7342 \\
\hline 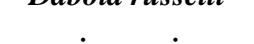 & 11293 & 9544 & 9806 & 25230 & 10156 & 6580 & 7014 & 12459 & 8259 & 20711 & 10597 & 7171 \\
\hline & 12000 & 10099 & 9225 & 24242 & 9485 & 7125 & 6704 & 12607 & 8160 & 18441 & 10505 & 7698 \\
\hline & 11995 & 9764 & 9201 & 24198 & 9631 & 7178 & 6607 & 12091 & 9125 & 16642 & 10264 & 7245 \\
\hline & 10844 & 9505 & 8523 & 25590 & 9174 & 6797 & 7224 & 12339 & 8325 & 20316 & 10753 & 6433 \\
\hline & 11108 & 9434 & 8410 & 25010 & 9439 & 6466 & 6416 & 11049 & 7889 & 19858 & 10448 & 6641 \\
\hline & 11877 & 9400 & 9392 & 23400 & 9685 & 6867 & 7640 & 12781 & 8579 & 16696 & 10459 & 7794 \\
\hline & 11455 & 9540 & 8578 & 25881 & 9268 & 6716 & 6176 & 12848 & 7951 & 20762 & 10276 & 6671 \\
\hline & 12400 & 9758 & 8742 & 23536 & 10882 & 6987 & 7369 & 12846 & 8964 & 19451 & 10870 & 7162 \\
\hline \multirow{14}{*}{ Naja atra } & 10274 & 9059 & 7411 & 18680 & 7010 & 5820 & 5347 & 12577 & 7478 & 16459 & 5824 & 7645 \\
\hline & 9778 & 8787 & 7428 & 18874 & 7173 & 5626 & 5472 & 11142 & 7195 & 16108 & 5467 & 7550 \\
\hline & 9712 & 8270 & 7435 & 17717 & 6739 & 5524 & 5325 & 11700 & 6861 & 14504 & 5159 & 7996 \\
\hline & 9608 & 8957 & 7510 & 18438 & 6518 & 5779 & 5643 & 11379 & 6954 & 14801 & 5202 & 7270 \\
\hline & 9564 & 9095 & 7218 & 18350 & 6868 & 5310 & 5752 & 11050 & 7411 & 15698 & 5474 & 7281 \\
\hline & 9225 & 8705 & 7276 & 18646 & 7012 & 5343 & 6421 & 10236 & 7353 & 16607 & 5571 & 8318 \\
\hline & 10272 & 8539 & 7915 & 17862 & 6941 & 5959 & 6437 & 12560 & 8113 & 15676 & 5373 & 7502 \\
\hline & 11086 & 8654 & 7995 & 18451 & 6831 & 5991 & 5474 & 11139 & 8014 & 14529 & 4716 & 6986 \\
\hline & 10536 & 8589 & 7336 & 18177 & 7133 & 6566 & 6038 & 9517 & 7244 & 15076 & 6129 & 7865 \\
\hline & 10648 & 8920 & 8065 & 19442 & 6952 & 6005 & 5940 & 11005 & 7792 & 15366 & 5863 & 7374 \\
\hline & 10683 & 8683 & 7947 & 18315 & 7038 & 6518 & 5950 & 11720 & 7563 & 16788 & 6029 & 7685 \\
\hline & 10825 & 8769 & 7996 & 18664 & 7222 & 5733 & 5865 & 12469 & 7482 & 15722 & 5275 & 7188 \\
\hline & 11007 & 9194 & 7977 & 17954 & 7227 & 5249 & 5689 & 10669 & 7096 & 15122 & 6094 & 7617 \\
\hline & 10285 & 8760 & 7980 & 18366 & 7177 & 5382 & 5820 & 10944 & 7934 & 15589 & 5408 & 7372 \\
\hline \multirow{7}{*}{ Agkistrodon Halys } & 10521 & 6845 & 8247 & 23531 & 8303 & 7195 & 5633 & 12771 & 7788 & 20088 & 8395 & 6620 \\
\hline & 9878 & 6938 & 7662 & 23536 & 7267 & 6494 & 5560 & 11804 & 7416 & 17257 & 8348 & 7323 \\
\hline & 10103 & 5986 & 8062 & 24747 & 8959 & 6180 & 5783 & 12174 & 7165 & 17629 & 7637 & 7311 \\
\hline & 9197 & 6200 & 7884 & 23296 & 8378 & 7142 & 5460 & 11989 & 7807 & 18780 & 8398 & 6362 \\
\hline & 9900 & 6253 & 8322 & 25717 & 9231 & 7430 & 5711 & 12300 & 7138 & 18064 & 8815 & 6649 \\
\hline & 10341 & 7359 & 8307 & 22503 & 8834 & 6829 & 5813 & 10387 & 7554 & 18715 & 8766 & 6675 \\
\hline & 10893 & 7642 & 8567 & 23970 & 7425 & 7930 & 6147 & 13055 & 8490 & 18866 & 8465 & 6811 \\
\hline
\end{tabular}




\begin{tabular}{|c|c|c|c|c|c|c|c|c|c|c|c|c|}
\hline & 11493 & 6961 & 8171 & 23160 & 9108 & 7131 & 6053 & 10893 & 7732 & 20656 & 8669 & 7166 \\
\hline & 10424 & 7102 & 7634 & 23809 & 7938 & 6997 & 5872 & 12949 & 7534 & 18253 & 8036 & 6728 \\
\hline & 10833 & 8015 & 8315 & 24720 & 7431 & 7048 & 6842 & 12614 & 8279 & 18825 & 8317 & 5950 \\
\hline & 10925 & 6958 & 8420 & 25033 & 8249 & 8149 & 6208 & 11458 & 7885 & 19202 & 8798 & 6247 \\
\hline & 12054 & 7999 & 8063 & 24303 & 7462 & 6981 & 6939 & 12891 & 8345 & 17489 & 8552 & 6562 \\
\hline & 10996 & 7441 & 7841 & 25004 & 7410 & 7450 & 5514 & 10282 & 7337 & 18432 & 7746 & 7105 \\
\hline & 11746 & 6824 & 8033 & 24024 & 8126 & 5673 & 6242 & 11605 & 7962 & 18597 & 8370 & 5950 \\
\hline \multirow{14}{*}{$\begin{array}{c}\text { Ophiophagus } \\
\text { hannah }\end{array}$} & 10060 & 7693 & 7760 & 20921 & 8303 & 10710 & 7563 & 11945 & 8530 & 17798 & 9459 & 8209 \\
\hline & 10459 & 7510 & 8196 & 23399 & 7267 & 9326 & 7880 & 12438 & 8511 & 18409 & 9580 & 7997 \\
\hline & 10603 & 8055 & 8038 & 22391 & 8959 & 10564 & 7993 & 12325 & 8682 & 18708 & 9245 & 7191 \\
\hline & 10131 & 7750 & 7956 & 21981 & 8378 & 9495 & 7128 & 12096 & 8075 & 15898 & 9483 & 7166 \\
\hline & 11139 & 7841 & 8221 & 22930 & 9231 & 10285 & 7968 & 11385 & 8213 & 19055 & 9131 & 7380 \\
\hline & 11793 & 7747 & 9156 & 24172 & 8834 & 10359 & 7903 & 11327 & 8867 & 21613 & 9544 & 7035 \\
\hline & 11738 & 7877 & 7923 & 21782 & 7425 & 10237 & 7588 & 11112 & 8159 & 18278 & 9763 & 7784 \\
\hline & 11367 & 7929 & 8618 & 22528 & 9108 & 10778 & 7749 & 10617 & 8649 & 20318 & 9509 & 7534 \\
\hline & 9811 & 7747 & 8158 & 22183 & 7938 & 9631 & 8265 & 10659 & 8097 & 19409 & 9898 & 6342 \\
\hline & 11846 & 7667 & 7533 & 20573 & 7431 & 9883 & 7289 & 11358 & 8562 & 18020 & 9222 & 7735 \\
\hline & 9147 & 7609 & 6779 & 24403 & 8249 & 10884 & 7471 & 12161 & 9138 & 22162 & 8761 & 6487 \\
\hline & 10485 & 7980 & 7684 & 23123 & 7462 & 10460 & 7560 & 10839 & 8294 & 16901 & 9026 & 6349 \\
\hline & 9755 & 8006 & 6640 & 20494 & 7410 & 10109 & 6891 & 12156 & 7957 & 15600 & 9242 & 6343 \\
\hline & 10987 & 7722 & 6809 & 20218 & 8126 & 10648 & 7625 & 11586 & 8409 & 16528 & 9135 & 6537 \\
\hline \multirow{14}{*}{ Control } & 10352 & 8849 & 6672 & 15087 & 6920 & 5457 & 6638 & 13285 & 6743 & 18200 & 8014 & 5876 \\
\hline & 10148 & 9647 & 6834 & 13806 & 8361 & 5395 & 6516 & 12192 & 6627 & 18986 & 7926 & 5861 \\
\hline & 10062 & 9572 & 6815 & 13602 & 8224 & 4798 & 6387 & 12334 & 6723 & 18296 & 7948 & 6548 \\
\hline & 10221 & 8859 & 7467 & 13485 & 6949 & 4759 & 6576 & 12955 & 7161 & 17677 & 8049 & 6338 \\
\hline & 10785 & 9022 & 7782 & 15070 & 7840 & 4676 & 6867 & 12662 & 7310 & 18172 & 8296 & 5890 \\
\hline & 10995 & 9502 & 7814 & 16375 & 8267 & 5112 & 6857 & 12003 & 7471 & 18050 & 8703 & 5581 \\
\hline & 11346 & 9596 & 8325 & 17435 & 9304 & 5487 & 6890 & 12922 & 8084 & 18364 & 8945 & 5794 \\
\hline & 11576 & 10606 & 7981 & 17632 & 9277 & 5466 & 7293 & 11576 & 8012 & 18217 & 8601 & 5384 \\
\hline & 11659 & 10007 & 8041 & 17568 & 9401 & 5487 & 7284 & 12053 & 8068 & 18313 & 8916 & 5305 \\
\hline & 11259 & 10627 & 7956 & 16828 & 9184 & 5417 & 7583 & 11229 & 7997 & 18413 & 8934 & 5236 \\
\hline & 11353 & 10225 & 7557 & 16464 & 8617 & 5322 & 7270 & 12030 & 7823 & 18719 & 8453 & 5251 \\
\hline & 11216 & 9470 & 7659 & 17672 & 9168 & 5947 & 6637 & 12368 & 7260 & 19179 & 8416 & 5314 \\
\hline & 11287 & 8858 & 7310 & 16120 & 9250 & 5365 & 6671 & 11923 & 7831 & 18734 & 8439 & 5914 \\
\hline & 11569 & 9103 & 7065 & 16521 & 8091 & 5505 & 7555 & 12284 & 8167 & 19957 & 9326 & 5955 \\
\hline & \multicolumn{12}{|c|}{ FITC } \\
\hline & $\mathrm{CH} 1$ & $\mathrm{CH} 2$ & $\mathrm{CH} 3$ & $\mathrm{CH} 4$ & $\mathrm{CH} 5$ & CH6 & $\mathrm{CH} 1$ & $\mathrm{CH} 2$ & $\mathrm{CH} 3$ & $\mathrm{CH} 4$ & $\mathrm{CH} 5$ & CH6 \\
\hline & 302 & 302 & 302 & 302 & 302 & 302 & 365 & 365 & 365 & 365 & 365 & 365 \\
\hline & 450 & 480 & 505 & 535 & 570 & 605 & 450 & 480 & 505 & 535 & 570 & 605 \\
\hline & 10281 & 6425 & 11808 & 16122 & 8315 & 7636 & 8419 & 13023 & 16520 & 41301 & 15619 & 15285 \\
\hline
\end{tabular}




\begin{tabular}{|c|c|c|c|c|c|c|c|c|c|c|c|c|}
\hline \multirow{13}{*}{$\begin{array}{c}\text { Gloydius blomhoffi } \\
\text { siniticus }\end{array}$} & 10002 & 5775 & 11594 & 18292 & 8221 & 7975 & 8490 & 13098 & 16336 & 40346 & 15357 & 14634 \\
\hline & 10040 & 5816 & 11366 & 16628 & 8118 & 7540 & 8743 & 11317 & 16539 & 42260 & 15677 & 14568 \\
\hline & 9729 & 6619 & 11677 & 16746 & 8225 & 8333 & 7568 & 12072 & 16609 & 31623 & 15066 & 15833 \\
\hline & 9989 & 7501 & 11379 & 17009 & 8634 & 8398 & 7789 & 11487 & 17011 & 38291 & 15486 & 15219 \\
\hline & 10541 & 7173 & 11284 & 17181 & 8152 & 8688 & 9044 & 10523 & 17927 & 46840 & 15703 & 14776 \\
\hline & 12142 & 7572 & 11904 & 17373 & 8458 & 8736 & 8830 & 11305 & 16966 & 45745 & 16201 & 14654 \\
\hline & 12838 & 7378 & 10258 & 17513 & 8359 & 8410 & 9139 & 11034 & 16692 & 46170 & 16218 & 15142 \\
\hline & 12267 & 8067 & 11599 & 16747 & 8757 & 8524 & 8701 & 11815 & 17499 & 42216 & 15378 & 14023 \\
\hline & 11827 & 7588 & 11704 & 18214 & 8036 & 8706 & 9499 & 12577 & 16493 & 42889 & 16135 & 15276 \\
\hline & 11563 & 8079 & 12439 & 18068 & 8829 & 8849 & 9686 & 11855 & 17333 & 46779 & 15448 & 14892 \\
\hline & 11871 & 7598 & 11345 & 16882 & 8578 & 8386 & 9014 & 12191 & 17309 & 48198 & 16184 & 14937 \\
\hline & 12124 & 8102 & 10296 & 18090 & 8102 & 8649 & 9352 & 12001 & 17748 & 48070 & 16862 & 16020 \\
\hline & 12213 & 8407 & 11161 & 17699 & 8154 & 9068 & 9477 & 13080 & 16836 & 44607 & 16965 & 15195 \\
\hline \multirow{14}{*}{$\begin{array}{c}\text { Bungarus } \\
\text { multicinctus }\end{array}$} & 10140 & 6150 & 10321 & 13246 & 5406 & 7223 & 6783 & 13791 & 14971 & 25129 & 10825 & 12819 \\
\hline & 10015 & 5649 & 10273 & 13911 & 5941 & 7970 & 5640 & 13382 & 15639 & 26488 & 11810 & 13183 \\
\hline & 10407 & 6899 & 10886 & 14634 & 6950 & 7905 & 7209 & 14138 & 18347 & 28442 & 11394 & 12700 \\
\hline & 10050 & 6582 & 13367 & 17677 & 6952 & 8157 & 6334 & 13323 & 16630 & 28879 & 12347 & 12760 \\
\hline & 10447 & 7586 & 12694 & 17280 & 6632 & 7799 & 5924 & 13176 & 16005 & 29637 & 12057 & 12850 \\
\hline & 11597 & 6851 & 12100 & 16794 & 7235 & 8723 & 7215 & 12975 & 19207 & 26022 & 11850 & 13738 \\
\hline & 11699 & 6989 & 13496 & 18492 & 6836 & 8074 & 6931 & 13751 & 18782 & 27142 & 11025 & 12510 \\
\hline & 10759 & 7169 & 13185 & 17378 & 7264 & 8657 & 6679 & 13918 & 19208 & 25258 & 11022 & 12193 \\
\hline & 11425 & 7536 & 13756 & 18359 & 7311 & 8273 & 7498 & 12698 & 20124 & 29488 & 11121 & 12068 \\
\hline & 10548 & 6959 & 14127 & 19028 & 7432 & 8381 & 7755 & 12688 & 21276 & 26257 & 11687 & 12918 \\
\hline & 10367 & 7471 & 14333 & 18594 & 7144 & 7958 & 7801 & 13106 & 21921 & 25021 & 10841 & 12457 \\
\hline & 10726 & 6382 & 14698 & 19017 & 7378 & 7192 & 6711 & 13452 & 19544 & 26035 & 11845 & 13263 \\
\hline & 11086 & 7423 & 14792 & 18013 & 7719 & 7700 & 7043 & 13820 & 21261 & 23848 & 11742 & 13000 \\
\hline & 10660 & 7988 & 14211 & 17526 & 6958 & 8384 & 6916 & 13050 & 18450 & 25311 & 10974 & 12949 \\
\hline \multirow{14}{*}{$\begin{array}{c}\text { Deinagkistrodon } \\
\text { acutus }\end{array}$} & 10735 & 7656 & 11102 & 16874 & 6123 & 7239 & 7569 & 11988 & 16885 & 32706 & 14293 & 14244 \\
\hline & 10037 & 7618 & 11079 & 18160 & 6375 & 7625 & 7729 & 11632 & 17632 & 35044 & 13945 & 14368 \\
\hline & 10145 & 7214 & 10031 & 16339 & 6872 & 7744 & 7868 & 11808 & 16929 & 34184 & 13990 & 15611 \\
\hline & 10415 & 7567 & 11678 & 16899 & 6549 & 8137 & 6473 & 12889 & 17022 & 34602 & 13642 & 15473 \\
\hline & 10310 & 7625 & 11246 & 16146 & 6264 & 8099 & 7150 & 11277 & 17188 & 31461 & 14150 & 15202 \\
\hline & 10658 & 8295 & 11059 & 17290 & 6499 & 7674 & 8301 & 10977 & 17462 & 37658 & 13470 & 14982 \\
\hline & 11149 & 8350 & 11241 & 17242 & 6866 & 7837 & 7325 & 12201 & 18783 & 37984 & 14636 & 15516 \\
\hline & 10864 & 7647 & 11545 & 17152 & 6839 & 7300 & 8152 & 11671 & 18097 & 36837 & 13971 & 15196 \\
\hline & 11722 & 7409 & 11055 & 17909 & 6847 & 8168 & 8419 & 13256 & 18377 & 33471 & 13701 & 15340 \\
\hline & 11262 & 7467 & 11359 & 17590 & 6171 & 7318 & 8230 & 11789 & 17170 & 32656 & 14030 & 14826 \\
\hline & 10120 & 7956 & 10907 & 18895 & 6492 & 7729 & 8971 & 11955 & 18001 & 34394 & 13039 & 13237 \\
\hline & 10268 & 7450 & 11424 & 16872 & 6215 & 7793 & 8123 & 13606 & 17474 & 35657 & 14295 & 15322 \\
\hline & 10677 & 7601 & 11796 & 18083 & 6866 & 7465 & 8426 & 13062 & 17811 & 32543 & 14224 & 14967 \\
\hline & 11653 & 7280 & 10602 & 18971 & 6467 & 7452 & 8871 & 11173 & 17890 & 35269 & 13700 & 14515 \\
\hline
\end{tabular}




\begin{tabular}{|c|c|c|c|c|c|c|c|c|c|c|c|c|}
\hline \multirow{14}{*}{$\begin{array}{c}\text { Daboia russelii } \\
\text { siamensis }\end{array}$} & 10583 & 7207 & 11352 & 19558 & 7593 & 8449 & 8761 & 12347 & 17574 & 25498 & 15070 & 13743 \\
\hline & 9597 & 6638 & 11051 & 16729 & 7618 & 8626 & 9131 & 12875 & 17322 & 24086 & 15139 & 13942 \\
\hline & 9546 & 8457 & 10691 & 16056 & 7717 & 8478 & 8944 & 13917 & 16271 & 26798 & 14413 & 13821 \\
\hline & 10292 & 6553 & 11878 & 16821 & 7642 & 8550 & 7480 & 12128 & 16764 & 24490 & 15442 & 13502 \\
\hline & 10578 & 7417 & 11529 & 18472 & 7954 & 8803 & 8129 & 11857 & 17584 & 28243 & 16281 & 13343 \\
\hline & 11519 & 8323 & 10543 & 18920 & 7596 & 8701 & 9602 & 12140 & 17230 & 28491 & 15917 & 12861 \\
\hline & 10491 & 8306 & 11389 & 18878 & 7924 & 8501 & 10061 & 13161 & 16618 & 29337 & 16232 & 13631 \\
\hline & 11060 & 8281 & 11456 & 18256 & 8083 & 8534 & 9633 & 13602 & 16866 & 29978 & 17402 & 13459 \\
\hline & 11130 & 10102 & 10045 & 18507 & 7971 & 8540 & 10389 & 14917 & 17894 & 24586 & 17759 & 13844 \\
\hline & 10660 & 8662 & 9890 & 19815 & 7319 & 8692 & 10072 & 13270 & 18034 & 28195 & 16296 & 13733 \\
\hline & 10700 & 8473 & 11575 & 17145 & 7470 & 8562 & 9676 & 13719 & 18184 & 26784 & 15572 & 13374 \\
\hline & 11265 & 8048 & 10638 & 17168 & 7741 & 8700 & 9724 & 13478 & 18166 & 26611 & 16968 & 12924 \\
\hline & 11406 & 8657 & 10211 & 17225 & 8041 & 8530 & 8768 & 13653 & 16990 & 27520 & 15465 & 13512 \\
\hline & 10619 & 9563 & 11258 & 18006 & 7676 & 8380 & 9439 & 12684 & 17513 & 26547 & 16536 & 13443 \\
\hline \multirow{14}{*}{ Naja atra } & 9972 & 7960 & 10196 & 16012 & 5207 & 7279 & 6811 & 12526 & 14835 & 29939 & 12162 & 11398 \\
\hline & 10149 & 7846 & 10117 & 15445 & 5676 & 7048 & 6270 & 12428 & 14204 & 29626 & 11962 & 11514 \\
\hline & 10451 & 7553 & 10403 & 17261 & 6247 & 8303 & 6416 & 11778 & 14298 & 29625 & 12299 & 10857 \\
\hline & 10721 & 7742 & 10731 & 16061 & 6679 & 7930 & 6916 & 12643 & 14269 & 28493 & 12505 & 10675 \\
\hline & 10662 & 7524 & 10300 & 16056 & 6399 & 8384 & 7062 & 11500 & 13995 & 28832 & 12477 & 10794 \\
\hline & 10486 & 7543 & 10995 & 15884 & 6174 & 7749 & 7132 & 12209 & 14985 & 29664 & 12268 & 11274 \\
\hline & 11017 & 7917 & 10528 & 16856 & 6591 & 7707 & 7563 & 12295 & 14566 & 30587 & 11493 & 12238 \\
\hline & 11156 & 8070 & 10848 & 17790 & 6305 & 7861 & 8065 & 11245 & 13851 & 29692 & 12123 & 11262 \\
\hline & 11148 & 7585 & 10319 & 16448 & 6165 & 8095 & 6856 & 12931 & 14002 & 28824 & 12915 & 10626 \\
\hline & 10530 & 7885 & 10164 & 16819 & 6049 & 7616 & 7916 & 11284 & 14699 & 32238 & 11889 & 11723 \\
\hline & 10051 & 7415 & 10282 & 15655 & 5976 & 7305 & 7444 & 12105 & 15398 & 29444 & 12333 & 11404 \\
\hline & 10538 & 7662 & 10498 & 16152 & 6051 & 7312 & 8155 & 13314 & 15043 & 28933 & 12348 & 10383 \\
\hline & 10591 & 7962 & 10612 & 16056 & 6370 & 7334 & 7586 & 12202 & 14339 & 26063 & 12172 & 10384 \\
\hline & 11693 & 7912 & 10112 & 16338 & 6456 & 7320 & 7329 & 12508 & 14337 & 29331 & 12386 & 11938 \\
\hline \multirow{11}{*}{ Agkistrodon Halys } & 10497 & 6908 & 14153 & 17592 & 7089 & 8221 & 8337 & 13924 & 17109 & 31390 & 13246 & 12219 \\
\hline & 10643 & 7735 & 13954 & 17798 & 7157 & 8359 & 8657 & 13287 & 17401 & 30523 & 15113 & 12190 \\
\hline & 10427 & 6921 & 14056 & 17937 & 7212 & 8499 & 8498 & 13304 & 19366 & 33780 & 14930 & 11073 \\
\hline & 10578 & 8010 & 13630 & 18086 & 7218 & 8682 & 7707 & 13861 & 16914 & 29225 & 13255 & 12097 \\
\hline & 10003 & 9066 & 13691 & 17328 & 7296 & 8583 & 8032 & 12162 & 17946 & 30096 & 13361 & 12258 \\
\hline & 10032 & 7618 & 13498 & 17588 & 7392 & 8861 & 9128 & 12190 & 19577 & 33362 & 14747 & 12729 \\
\hline & 11260 & 7772 & 13794 & 17823 & 7508 & 8903 & 9107 & 12681 & 18948 & 35744 & 16659 & 13900 \\
\hline & 10962 & 9056 & 14479 & 18248 & 7724 & 8244 & 9268 & 13356 & 20696 & 36580 & 16183 & 13077 \\
\hline & 11035 & 8202 & 13702 & 16856 & 6985 & 7846 & 9402 & 13366 & 19964 & 36025 & 15581 & 14011 \\
\hline & 11083 & 8055 & 13551 & 17099 & 7743 & 8090 & 9761 & 13730 & 19135 & 33348 & 14913 & 13348 \\
\hline & 10429 & 7500 & 14766 & 18312 & 6723 & 8860 & 8856 & 13597 & 21274 & 37182 & 16629 & 13223 \\
\hline \multirow{2}{*}{ Agkistrodon Halys } & 9991 & 7080 & 15410 & 17713 & 7208 & 8813 & 8817 & 13689 & 19928 & 36420 & 16727 & 14424 \\
\hline & 10375 & 7453 & 14627 & 18847 & 6944 & 8262 & 7912 & 13420 & 19566 & 34573 & 15969 & 13824 \\
\hline
\end{tabular}




\begin{tabular}{|c|c|c|c|c|c|c|c|c|c|c|c|c|}
\hline & 10738 & 8284 & 15232 & 17994 & 7058 & 8021 & 8748 & 12071 & 19504 & 33295 & 16358 & 13118 \\
\hline \multirow{14}{*}{$\begin{array}{c}\text { Ophiophagus } \\
\text { hannah }\end{array}$} & 11771 & 6296 & 12042 & 16909 & 6223 & 8501 & 8493 & 12555 & 19103 & 34479 & 15242 & 13909 \\
\hline & 10769 & 6286 & 11990 & 17182 & 6635 & 8606 & 8564 & 13068 & 18862 & 32882 & 14671 & 13800 \\
\hline & 11200 & 6201 & 12439 & 17495 & 6693 & 8547 & 8431 & 12657 & 18360 & 35095 & 16207 & 14303 \\
\hline & 11342 & 6691 & 12871 & 18125 & 7258 & 8992 & 8293 & 14030 & 18485 & 31582 & 16399 & 13674 \\
\hline & 11527 & 6272 & 13638 & 18469 & 7195 & 8804 & 8616 & 12999 & 17585 & 32588 & 14551 & 14043 \\
\hline & 11672 & 6483 & 13688 & 18270 & 6535 & 9320 & 8583 & 11779 & 19503 & 36636 & 14867 & 15362 \\
\hline & 12469 & 6597 & 14130 & 17376 & 6761 & 8537 & 9347 & 12042 & 18605 & 34301 & 16518 & 14805 \\
\hline & 12469 & 6475 & 13842 & 17803 & 6848 & 8854 & 8931 & 13495 & 19662 & 39281 & 16114 & 15541 \\
\hline & 12446 & 6478 & 14069 & 19227 & 6229 & 8915 & 8557 & 12920 & 19353 & 39678 & 17389 & 15780 \\
\hline & 11973 & 6243 & 14713 & 17955 & 6082 & 8420 & 9075 & 12734 & 18641 & 37909 & 18124 & 14525 \\
\hline & 12421 & 6365 & 14391 & 18194 & 6558 & 8380 & 8950 & 12831 & 18202 & 34014 & 17255 & 14179 \\
\hline & 11910 & 6359 & 13907 & 18291 & 6599 & 8497 & 8868 & 13426 & 17783 & 36948 & 16022 & 14461 \\
\hline & 12108 & 6324 & 13725 & 17884 & 6076 & 9101 & 9543 & 11562 & 18266 & 36365 & 17299 & 14957 \\
\hline & 11761 & 6248 & 13610 & 18030 & 6289 & 8013 & 9776 & 11309 & 17849 & 34681 & 16669 & 12618 \\
\hline \multirow{18}{*}{ Control } & 11553 & 6324 & 10718 & 15583 & 5535 & 7763 & 6715 & 10087 & 18373 & 27155 & 14328 & 12364 \\
\hline & 10467 & 6773 & 10657 & 13680 & 5611 & 7797 & 6776 & 10492 & 17629 & 24625 & 14110 & 12333 \\
\hline & 11646 & 5784 & 10893 & 14275 & 5871 & 8001 & 6018 & 10529 & 18780 & 25296 & 14300 & 12680 \\
\hline & 10521 & 6821 & 10802 & 13653 & 5472 & 8163 & 6013 & 9848 & 18064 & 25600 & 14511 & 12949 \\
\hline & 10955 & 7309 & 10303 & 13529 & 5672 & 8268 & 6295 & 8787 & 18715 & 25070 & 14576 & 12371 \\
\hline & 12179 & 6679 & 10584 & 15156 & 5944 & 8271 & 6770 & 8431 & 18866 & 28795 & 13933 & 12999 \\
\hline & 12359 & 6647 & 10449 & 15139 & 5433 & 8360 & 7736 & 9575 & 18488 & 30669 & 14641 & 12615 \\
\hline & 11591 & 7542 & 10872 & 15283 & 5760 & 8189 & 6930 & 9543 & 18253 & 34417 & 14615 & 11937 \\
\hline & 11181 & 6687 & 10023 & 14960 & 5340 & 8212 & 6885 & 9667 & 18825 & 30132 & 13869 & 12414 \\
\hline & 11743 & 6764 & 10528 & 15114 & 5842 & 8264 & 6642 & 10553 & 19202 & 34106 & 14715 & 11879 \\
\hline & 12448 & 6908 & 10026 & 15012 & 6087 & 8382 & 7786 & 10788 & 18632 & 29936 & 14117 & 12612 \\
\hline & 12720 & 6262 & 11320 & 15856 & 5791 & 8431 & 7686 & 10294 & 17489 & 31792 & 15237 & 13028 \\
\hline & 11552 & 6966 & 11971 & 15862 & 5681 & 8286 & 6944 & 10765 & 18432 & 30705 & 13767 & 11704 \\
\hline & 11909 & 7332 & 11030 & 13895 & 5972 & 8284 & 6438 & 10230 & 18597 & 29920 & 14830 & 11893 \\
\hline & \multicolumn{12}{|c|}{ SRB } \\
\hline & $\mathrm{CH} 1$ & $\mathrm{CH} 2$ & $\mathrm{CH} 3$ & $\mathrm{CH} 4$ & CH5 & CH6 & CH1 & $\mathrm{CH} 2$ & $\mathrm{CH} 3$ & $\mathrm{CH} 4$ & CH5 & CH6 \\
\hline & 302 & 302 & 302 & 302 & 302 & 302 & 365 & 365 & 365 & 365 & 365 & 365 \\
\hline & 450 & 480 & 505 & 535 & 570 & 605 & 450 & 480 & 505 & 535 & 570 & 605 \\
\hline \multirow{8}{*}{$\begin{array}{c}\text { Gloydius blomhoffi } \\
\text { siniticus }\end{array}$} & 10940 & 8029 & 4912 & 6288 & 20942 & 39224 & 7711 & 13025 & 4974 & 5919 & 21360 & 33234 \\
\hline & 9878 & 7183 & 4800 & 5483 & 22078 & 36381 & 7844 & 13203 & 5201 & 6114 & 18225 & 29425 \\
\hline & 9956 & 7010 & 4947 & 5928 & 20946 & 36239 & 7970 & 13416 & 4682 & 6206 & 19195 & 31831 \\
\hline & 10459 & 7095 & 5042 & 5813 & 21848 & 32582 & 6980 & 12509 & 4942 & 6431 & 18966 & 33402 \\
\hline & 10388 & 7639 & 5278 & 5881 & 20942 & 35251 & 7114 & 11662 & 4705 & 6395 & 19237 & 35890 \\
\hline & 11401 & 7278 & 5302 & 5917 & 20414 & 36941 & 8130 & 11517 & 5124 & 6617 & 20184 & 40228 \\
\hline & 10849 & 7411 & 5413 & 5412 & 23298 & 35690 & 8401 & 12662 & 5273 & 6289 & 18315 & 34838 \\
\hline & 12081 & 7163 & 5025 & 6132 & 20029 & 38989 & 8664 & 13319 & 4987 & 6789 & 19597 & 36466 \\
\hline
\end{tabular}




\begin{tabular}{|c|c|c|c|c|c|c|c|c|c|c|c|c|}
\hline & 11871 & 7216 & 4945 & 5674 & 23511 & 35215 & 8815 & 14180 & 4770 & 6791 & 20504 & 32845 \\
\hline & 11201 & 6955 & 4692 & 5884 & 24689 & 37799 & 8942 & 13050 & 4296 & 6925 & 21176 & 35089 \\
\hline & 11207 & 6819 & 5021 & 5432 & 24585 & 34951 & 8098 & 13244 & 4792 & 6703 & 19397 & 36963 \\
\hline & 10301 & 7482 & 4991 & 6374 & 23195 & 35342 & 8244 & 13676 & 4718 & 6664 & 17789 & 34203 \\
\hline & 11176 & 7520 & 4713 & 6065 & 24864 & 35044 & 7988 & 13578 & 4837 & 6144 & 18154 & 33960 \\
\hline & 10828 & 7378 & 5290 & 5407 & 20549 & 33540 & 8369 & 11769 & 4982 & 6984 & 18362 & 34424 \\
\hline \multirow{14}{*}{$\begin{array}{c}\text { Bungarus } \\
\text { multicinctus }\end{array}$} & 11293 & 7676 & 4487 & 10856 & 26222 & 18925 & 7800 & 15235 & 4871 & 7803 & 40226 & 36080 \\
\hline & 11434 & 7506 & 4813 & 11338 & 25654 & 18837 & 7970 & 14600 & 4743 & 7582 & 41070 & 33476 \\
\hline & 10422 & 7814 & 5190 & 11096 & 26315 & 20997 & 8129 & 16122 & 4716 & 7249 & 39370 & 38554 \\
\hline & 11007 & 7441 & 5507 & 10626 & 28783 & 19182 & 7032 & 16885 & 4736 & 7676 & 34917 & 35500 \\
\hline & 11142 & 8012 & 5243 & 10731 & 28843 & 17652 & 7008 & 14733 & 4559 & 7360 & 36195 & 34982 \\
\hline & 12312 & 8011 & 5506 & 11543 & 27263 & 21779 & 8470 & 14908 & 4730 & 8009 & 39349 & 37755 \\
\hline & 12038 & 7689 & 5183 & 10914 & 30992 & 17487 & 7982 & 15086 & 4640 & 8061 & 42086 & 33618 \\
\hline & 12210 & 7985 & 5244 & 11051 & 24845 & 19022 & 8616 & 13994 & 4531 & 8163 & 42970 & 37219 \\
\hline & 11768 & 7754 & 5364 & 11504 & 21482 & 19576 & 8889 & 15065 & 4830 & 7287 & 43660 & 35932 \\
\hline & 11463 & 7782 & 5382 & 11646 & 23846 & 19808 & 8974 & 17044 & 4690 & 7355 & 45101 & 33287 \\
\hline & 10751 & 7978 & 5135 & 11434 & 25152 & 21511 & 8731 & 17575 & 4633 & 7483 & 44299 & 32623 \\
\hline & 11582 & 7862 & 5310 & 11545 & 25261 & 17355 & 8636 & 16638 & 4283 & 7172 & 44594 & 33609 \\
\hline & 11819 & 7835 & 5420 & 12858 & 24093 & 18267 & 8355 & 14425 & 4558 & 7436 & 43628 & 31890 \\
\hline & 11369 & 7487 & 5159 & 13402 & 26170 & 20256 & 8804 & 13106 & 4864 & 7473 & 44615 & 31563 \\
\hline \multirow{14}{*}{$\begin{array}{c}\text { Deinagkistrodon } \\
\text { acutus }\end{array}$} & 10821 & 8074 & 4625 & 8109 & 29379 & 21143 & 6878 & 12973 & 4697 & 7203 & 24228 & 39936 \\
\hline & 10980 & 7611 & 4408 & 7879 & 27724 & 25878 & 7278 & 14516 & 4713 & 7506 & 23312 & 35269 \\
\hline & 11122 & 7737 & 4375 & 7930 & 28494 & 22239 & 6797 & 13211 & 4400 & 7096 & 25273 & 38627 \\
\hline & 10601 & 7734 & 4495 & 7349 & 28796 & 25790 & 7349 & 13329 & 4639 & 7526 & 22981 & 39159 \\
\hline & 10587 & 7912 & 4634 & 7671 & 27238 & 23132 & 7980 & 13742 & 4451 & 7165 & 22843 & 40683 \\
\hline & 11160 & 7704 & 4930 & 7512 & 28558 & 23182 & 7934 & 11329 & 4333 & 7221 & 25912 & 35951 \\
\hline & 12004 & 7787 & 4745 & 8148 & 28850 & 21151 & 7266 & 11504 & 4573 & 7199 & 22473 & 39745 \\
\hline & 11661 & 7319 & 4576 & 8304 & 29479 & 23437 & 7415 & 11604 & 4432 & 7931 & 24026 & 38583 \\
\hline & 11622 & 7667 & 4583 & 7318 & 28491 & 24513 & 7068 & 12596 & 4333 & 7161 & 23551 & 37990 \\
\hline & 11035 & 7629 & 4498 & 7895 & 28632 & 24380 & 6953 & 13364 & 4244 & 7879 & 23090 & 34564 \\
\hline & 10682 & 8028 & 4461 & 7879 & 27554 & 24122 & 7677 & 12839 & 4028 & 7478 & 22846 & 35066 \\
\hline & 11164 & 7716 & 4396 & 7894 & 27921 & 22121 & 8212 & 12999 & 4367 & 7712 & 22748 & 34843 \\
\hline & 11402 & 7937 & 4921 & 7616 & 29789 & 22200 & 7709 & 13614 & 4539 & 7512 & 21933 & 35772 \\
\hline & 12388 & 8297 & 4678 & 7802 & 29389 & 22963 & 8103 & 12109 & 4332 & 7572 & 23694 & 35569 \\
\hline \multirow{7}{*}{$\begin{array}{c}\text { Daboia russelii } \\
\text { siamensis }\end{array}$} & 6298 & 7758 & 3998 & 6855 & 31108 & 33967 & 7229 & 13406 & 4559 & 6066 & 21604 & 37426 \\
\hline & 6172 & 7843 & 4178 & 6962 & 30562 & 33768 & 6438 & 15701 & 4435 & 6784 & 21060 & 35774 \\
\hline & 6602 & 7210 & 4297 & 6153 & 31493 & 35803 & 7203 & 15692 & 4332 & 6719 & 21843 & 36031 \\
\hline & 6291 & 7180 & 4182 & 6067 & 29662 & 37378 & 7679 & 14988 & 4470 & 6448 & 22007 & 35749 \\
\hline & 7126 & 7672 & 4083 & 6685 & 33015 & 37771 & 7967 & 13619 & 4240 & 6326 & 21189 & 34030 \\
\hline & 6507 & 7797 & 4132 & 7008 & 34465 & 37756 & 7571 & 14277 & 4689 & 6434 & 24185 & 34046 \\
\hline & 7546 & 7459 & 4089 & 7439 & 34334 & 34996 & 6237 & 13967 & 4241 & 6986 & 24264 & 33422 \\
\hline
\end{tabular}




\begin{tabular}{|c|c|c|c|c|c|c|c|c|c|c|c|c|}
\hline & 6813 & 7729 & 4178 & 7147 & 29960 & 35581 & 6645 & 14125 & 4537 & 6524 & 23268 & 34968 \\
\hline & 7338 & 7808 & 3985 & 6594 & 29391 & 36767 & 6783 & 13205 & 4532 & 6305 & 23364 & 34689 \\
\hline & 7065 & 7581 & 4173 & 6715 & 32716 & 37515 & 7723 & 13991 & 4555 & 6092 & 21594 & 35781 \\
\hline & 7679 & 7862 & 4264 & 7123 & 28940 & 35345 & 7544 & 15013 & 4781 & 6150 & 22790 & 34109 \\
\hline & 6998 & 7315 & 4201 & 7005 & 31308 & 37754 & 8547 & 15197 & 4375 & 6936 & 20854 & 37898 \\
\hline & 7004 & 7416 & 4199 & 7015 & 34134 & 35765 & 7754 & 15127 & 4416 & 6337 & 21669 & 38463 \\
\hline & 7117 & 7475 & 4152 & 6894 & 34977 & 33075 & 7943 & 13144 & 4459 & 6982 & 20511 & 38718 \\
\hline \multirow{14}{*}{ Naja atra } & 6892 & 8005 & 4171 & 7130 & 39862 & 29787 & 6998 & 13067 & 4536 & 6947 & 23412 & 31623 \\
\hline & 6831 & 7990 & 4027 & 5962 & 39200 & 29931 & 6831 & 14815 & 4146 & 5996 & 23874 & 30992 \\
\hline & 6459 & 7215 & 4136 & 6539 & 35621 & 28975 & 6321 & 15811 & 4444 & 5854 & 22387 & 32282 \\
\hline & 6760 & 7863 & 4181 & 6949 & 40292 & 30531 & 6894 & 14819 & 4119 & 7032 & 21461 & 31002 \\
\hline & 6993 & 7890 & 4273 & 7502 & 41420 & 28817 & 7331 & 13385 & 4199 & 5883 & 23275 & 30357 \\
\hline & 7296 & 7980 & 4116 & 7372 & 40375 & 28176 & 6876 & 14189 & 4363 & 6664 & 25197 & 29984 \\
\hline & 7605 & 7459 & 4326 & 7866 & 40782 & 29114 & 6870 & 13768 & 4295 & 7119 & 22693 & 30315 \\
\hline & 7985 & 7884 & 4376 & 7818 & 40463 & 26877 & 6841 & 13988 & 4660 & 7611 & 24889 & 31954 \\
\hline & 7926 & 7607 & 4511 & 7570 & 41280 & 28095 & 6402 & 13468 & 4805 & 7817 & 23812 & 29894 \\
\hline & 8176 & 7768 & 4477 & 7838 & 40844 & 29240 & 6715 & 15474 & 4814 & 7656 & 24482 & 32686 \\
\hline & 6880 & 7929 & 4342 & 7391 & 38945 & 30482 & 7170 & 15667 & 4528 & 7237 & 24794 & 34108 \\
\hline & 7256 & 7699 & 4389 & 7322 & 45157 & 30293 & 6791 & 15067 & 4416 & 7076 & 24472 & 33941 \\
\hline & 8157 & 7277 & 4414 & 7112 & 38555 & 28737 & 7152 & 14265 & 4817 & 6865 & 25570 & 31612 \\
\hline & 8081 & 7801 & 4664 & 7017 & 44152 & 29290 & 7214 & 12813 & 4416 & 7738 & 24975 & 35560 \\
\hline \multirow{14}{*}{ Agkistrodon Halys } & 6100 & 7397 & 3778 & 5300 & 26897 & 38229 & 6632 & 13155 & 4040 & 5081 & 20355 & 35197 \\
\hline & 6447 & 7148 & 4056 & 4992 & 27349 & 38798 & 6113 & 15017 & 4191 & 5081 & 19805 & 33147 \\
\hline & 6192 & 7111 & 3896 & 5713 & 30785 & 35205 & 6529 & 13072 & 3699 & 5627 & 20426 & 32884 \\
\hline & 6305 & 7480 & 3740 & 5898 & 29842 & 38537 & 6311 & 14603 & 3733 & 5582 & 19810 & 34043 \\
\hline & 5860 & 7258 & 3886 & 5769 & 29725 & 36427 & 6350 & 12792 & 3453 & 5246 & 20897 & 34115 \\
\hline & 6202 & 7984 & 3790 & 5833 & 31048 & 36559 & 7139 & 11568 & 3458 & 5844 & 20185 & 34956 \\
\hline & 6054 & 7569 & 3772 & 5550 & 28878 & 37450 & 6183 & 13282 & 3405 & 5392 & 19551 & 34004 \\
\hline & 6658 & 7338 & 3640 & 5409 & 26127 & 39824 & 6225 & 13240 & 3632 & 6113 & 20322 & 33198 \\
\hline & 6991 & 7920 & 3990 & 5204 & 25211 & 38179 & 5827 & 13835 & 4107 & 6374 & 20462 & 33436 \\
\hline & 6737 & 7389 & 3820 & 5138 & 26220 & 37333 & 6006 & 14288 & 3824 & 6379 & 20668 & 36003 \\
\hline & 6244 & 7771 & 3701 & 5359 & 27678 & 37286 & 6373 & 14346 & 3572 & 5150 & 22051 & 33322 \\
\hline & 6484 & 7466 & 4021 & 4973 & 24280 & 39411 & 7154 & 14071 & 4128 & 5638 & 19010 & 33899 \\
\hline & 6349 & 7365 & 3935 & 5162 & 26507 & 43278 & 6098 & 12058 & 3765 & 5719 & 16968 & 33776 \\
\hline & 6159 & 7316 & 3862 & 5252 & 30425 & 42134 & 7241 & 12627 & 3662 & 5521 & 19808 & 34028 \\
\hline \multirow{6}{*}{$\begin{array}{c}\text { Ophiophagus } \\
\text { hannah }\end{array}$} & 8137 & 7289 & 4370 & 5822 & 31227 & 33696 & 6742 & 13246 & 4644 & 4986 & 19350 & 34533 \\
\hline & 7537 & 7390 & 4043 & 5898 & 32225 & 34412 & 5786 & 15783 & 4504 & 4977 & 19827 & 35214 \\
\hline & 8011 & 7611 & 4079 & 5462 & 31726 & 34825 & 6314 & 13881 & 4720 & 6056 & 18532 & 34384 \\
\hline & 8078 & 7635 & 4038 & 5875 & 33228 & 35884 & 6291 & 15790 & 4772 & 5878 & 19194 & 33548 \\
\hline & 7625 & 7685 & 4214 & 6354 & 35051 & 36095 & 6962 & 13367 & 4668 & 5966 & 18806 & 36592 \\
\hline & 8426 & 7326 & 4488 & 6560 & 34192 & 36635 & 6481 & 11852 & 4151 & 5342 & 19957 & 37189 \\
\hline
\end{tabular}




\begin{tabular}{|c|c|c|c|c|c|c|c|c|c|c|c|c|}
\hline & 8261 & 7594 & 4208 & 6014 & 34972 & 35949 & 6191 & 14074 & 4565 & 6093 & 21155 & 36921 \\
\hline & 8761 & 7279 & 4569 & 6032 & 33331 & 36178 & 6156 & 14094 & 4359 & 6096 & 19708 & 37164 \\
\hline & 8641 & 7345 & 4585 & 6027 & 32173 & 36834 & 5421 & 13021 & 4798 & 6504 & 19711 & 38952 \\
\hline & 8776 & 7470 & 4682 & 5719 & 30489 & 36002 & 5885 & 15559 & 4922 & 6693 & 20736 & 37100 \\
\hline & 7725 & 7548 & 4600 & 5659 & 29987 & 37254 & 6265 & 16076 & 4958 & 6175 & 19775 & 36740 \\
\hline & 8085 & 7708 & 4629 & 5746 & 30085 & 38049 & 6084 & 16024 & 4923 & 6282 & 18884 & 35956 \\
\hline & 8332 & 7451 & 4335 & 6528 & 31798 & 37653 & 6259 & 13917 & 5058 & 6474 & 18454 & 36508 \\
\hline & 8250 & 7690 & 4698 & 5736 & 33292 & 39497 & 6049 & 12911 & 5181 & 6617 & 19856 & 36323 \\
\hline \multirow{14}{*}{ Control } & 9553 & 7741 & 3711 & 3821 & 22195 & 39628 & 7072 & 13911 & 4197 & 5317 & 15076 & 28584 \\
\hline & 9667 & 7866 & 3780 & 3830 & 22505 & 37766 & 7402 & 16039 & 4323 & 5181 & 16265 & 29106 \\
\hline & 8481 & 7650 & 4040 & 4250 & 19803 & 39278 & 7241 & 16887 & 4128 & 6105 & 15223 & 29935 \\
\hline & 8608 & 8048 & 4142 & 4441 & 22455 & 40296 & 5864 & 15755 & 4332 & 6027 & 15443 & 29466 \\
\hline & 9785 & 7272 & 4479 & 4125 & 22918 & 40513 & 6525 & 14788 & 5003 & 5948 & 14612 & 29701 \\
\hline & 9205 & 7733 & 4162 & 5387 & 26239 & 39146 & 7283 & 14396 & 4602 & 6033 & 15362 & 27646 \\
\hline & 10279 & 7611 & 4450 & 5094 & 26130 & 37228 & 7277 & 14603 & 4949 & 6191 & 16708 & 29163 \\
\hline & 10130 & 7521 & 4232 & 5168 & 25683 & 35860 & 7924 & 13028 & 4761 & 6441 & 17001 & 29523 \\
\hline & 10324 & 7638 & 4650 & 5179 & 24090 & 35672 & 8205 & 14723 & 5192 & 6727 & 17594 & 30925 \\
\hline & 10280 & 7929 & 4719 & 5251 & 22164 & 38620 & 8219 & 16788 & 5449 & 6789 & 15718 & 32661 \\
\hline & 9950 & 7747 & 4642 & 5038 & 21142 & 37496 & 8431 & 16959 & 5369 & 6052 & 15643 & 29830 \\
\hline & 8899 & 8054 & 4758 & 4789 & 22801 & 43580 & 8572 & 16228 & 5313 & 6478 & 14995 & 30883 \\
\hline & 9955 & 7832 & 4368 & 4852 & 20913 & 39054 & 7784 & 12883 & 4973 & 6455 & 15860 & 32020 \\
\hline & 10487 & 8144 & 4421 & 4953 & 23250 & 38487 & 8413 & 12454 & 4936 & 6705 & 15750 & 32254 \\
\hline & \multicolumn{12}{|c|}{ TY } \\
\hline & $\mathrm{CH} 1$ & $\mathrm{CH} 2$ & $\mathrm{CH} 3$ & $\mathrm{CH} 4$ & CH5 & CH6 & CH1 & $\mathrm{CH} 2$ & $\mathrm{CH} 3$ & $\mathrm{CH} 4$ & CH5 & CH6 \\
\hline & 302 & 302 & 302 & 302 & 302 & 302 & 365 & 365 & 365 & 365 & 365 & 365 \\
\hline & 450 & 480 & 505 & 535 & 570 & 605 & 450 & 480 & 505 & 535 & 570 & 605 \\
\hline \multirow{15}{*}{$\begin{array}{c}\text { Gloydius blomhoffi } \\
\text { siniticus }\end{array}$} & 15632 & 8740 & 8143 & 9945 & 5607 & 6784 & 13244 & 14506 & 13454 & 9221 & 7639 & 9599 \\
\hline & 15952 & 8345 & 8861 & 10035 & 5397 & 7209 & 13350 & 14042 & 13492 & 9044 & 7974 & 9755 \\
\hline & 15700 & 8317 & 6953 & 9716 & 4591 & 7174 & 13180 & 14929 & 12557 & 9519 & 7404 & 9548 \\
\hline & 15218 & 8921 & 8913 & 10018 & 4513 & 7019 & 12903 & 15498 & 12077 & 9633 & 7188 & 9484 \\
\hline & 15451 & 10700 & 9062 & 9487 & 4455 & 7187 & 12604 & 14596 & 12480 & 9739 & 7373 & 9722 \\
\hline & 16455 & 10550 & 7332 & 9184 & 4946 & 7119 & 13575 & 12572 & 13081 & 9204 & 7766 & 9503 \\
\hline & 16205 & 9394 & 7391 & 10002 & 4841 & 7509 & 13779 & 14174 & 13004 & 9951 & 7954 & 9528 \\
\hline & 17328 & 9291 & 8457 & 10354 & 5014 & 7196 & 13487 & 15325 & 14014 & 10190 & 8059 & 9313 \\
\hline & 16536 & 8860 & 8595 & 9822 & 5054 & 7214 & 13206 & 14527 & 12564 & 10132 & 8515 & 9597 \\
\hline & 15959 & 9670 & 9294 & 10512 & 4946 & 7578 & 13729 & 14660 & 12651 & 10372 & 8633 & 9777 \\
\hline & 15607 & 8757 & 9809 & 10741 & 4853 & 6976 & 12756 & 14294 & 12257 & 10422 & 7853 & 9669 \\
\hline & 15513 & 9291 & 10253 & 10474 & 4573 & 7318 & 12689 & 13947 & 12742 & 9632 & 8082 & 9664 \\
\hline & 15773 & 10196 & 10766 & 10064 & 4572 & 7731 & 13033 & 13131 & 12477 & 9713 & 7196 & 9670 \\
\hline & 14685 & 10095 & 10745 & 10693 & 4617 & 7231 & 13531 & 13989 & 12618 & 10332 & 7227 & 10050 \\
\hline & 11315 & 6220 & 5200 & 9195 & 4916 & 6418 & 11138 & 14001 & 12070 & 11044 & 9454 & 9483 \\
\hline
\end{tabular}




\begin{tabular}{|c|c|c|c|c|c|c|c|c|c|c|c|c|}
\hline \multirow{13}{*}{$\begin{array}{c}\text { Bungarus } \\
\text { multicinctus }\end{array}$} & 10168 & 5625 & 5275 & 8407 & 5054 & 6530 & 10186 & 14739 & 11308 & 12022 & 9070 & 9331 \\
\hline & 10735 & 7040 & 5995 & 9310 & 4767 & 7045 & 9414 & 13933 & 11266 & 12199 & 9086 & 9244 \\
\hline & 9387 & 5919 & 5772 & 9118 & 4913 & 6558 & 9157 & 14139 & 10899 & 10495 & 8978 & 9227 \\
\hline & 9859 & 6809 & 5922 & 9227 & 4996 & 6850 & 9481 & 13992 & 11088 & 10286 & 8931 & 8847 \\
\hline & 9963 & 6905 & 5471 & 8618 & 5201 & 7037 & 9906 & 14012 & 11762 & 10594 & 9302 & 9202 \\
\hline & 10630 & 7400 & 5071 & 9864 & 4878 & 6687 & 9830 & 14848 & 11854 & 10904 & 9837 & 9500 \\
\hline & 11244 & 7190 & 4961 & 9688 & 5130 & 6939 & 11472 & 15288 & 11331 & 10621 & 9130 & 9142 \\
\hline & 11547 & 6198 & 4664 & 9577 & 4750 & 6666 & 10643 & 14180 & 11983 & 10266 & 9039 & 9585 \\
\hline & 11336 & 5676 & 4803 & 9513 & 4881 & 6556 & 10916 & 15353 & 11941 & 11104 & 9413 & 9055 \\
\hline & 11328 & 6426 & 4219 & 9945 & 4955 & 6742 & 11266 & 16793 & 11866 & 12318 & 9182 & 9108 \\
\hline & 10710 & 6535 & 4433 & 8840 & 5142 & 6727 & 10953 & 16815 & 11139 & 11708 & 9226 & 9343 \\
\hline & 11551 & 6443 & 4424 & 9892 & 4709 & 6689 & 10473 & 16254 & 11409 & 11080 & 8911 & 9421 \\
\hline & 10598 & 6648 & 5173 & 10155 & 4916 & 6654 & 11502 & 14635 & 11481 & 11661 & 9351 & 9435 \\
\hline \multirow{14}{*}{$\begin{array}{c}\text { Deinagkistrodon } \\
\text { Acutus }\end{array}$} & 19851 & 6265 & 8458 & 10696 & 6465 & 7577 & 18394 & 21040 & 13981 & 14618 & 9367 & 10158 \\
\hline & 19696 & 7343 & 8000 & 11107 & 6534 & 7629 & 17968 & 21508 & 13767 & 14495 & 9678 & 10281 \\
\hline & 19216 & 7281 & 7679 & 11439 & 6602 & 7020 & 18636 & 20443 & 13542 & 14669 & 10033 & 10295 \\
\hline & 20165 & 8377 & 7694 & 11209 & 6090 & 7864 & 18622 & 21055 & 11394 & 14660 & 10373 & 10380 \\
\hline & 21257 & 8220 & 7864 & 11825 & 5931 & 7534 & 17555 & 22323 & 13195 & 14544 & 10120 & 11138 \\
\hline & 19677 & 6769 & 8671 & 11251 & 6136 & 7638 & 18905 & 21018 & 13456 & 14592 & 10058 & 10917 \\
\hline & 21431 & 7716 & 8228 & 11872 & 6803 & 7977 & 18469 & 22909 & 14820 & 15108 & 10918 & 11185 \\
\hline & 21798 & 7938 & 7868 & 11325 & 6684 & 7701 & 19162 & 24461 & 15365 & 15303 & 10664 & 10162 \\
\hline & 19555 & 7850 & 8163 & 11296 & 7336 & 8280 & 17731 & 24905 & 15330 & 14757 & 10873 & 10236 \\
\hline & 19876 & 7383 & 8008 & 10843 & 6853 & 8103 & 18477 & 21956 & 15380 & 13793 & 10681 & 10324 \\
\hline & 19210 & 7250 & 7525 & 11214 & 6926 & 7907 & 18401 & 22069 & 14324 & 15592 & 10092 & 10158 \\
\hline & 20458 & 7234 & 8821 & 11403 & 6052 & 7550 & 19887 & 23644 & 14692 & 14606 & 10799 & 10693 \\
\hline & 19963 & 7213 & 7583 & 11440 & 6992 & 7161 & 19841 & 22115 & 14183 & 15896 & 10920 & 11058 \\
\hline & 20072 & 7153 & 7931 & 11315 & 5968 & 7511 & 19390 & 22086 & 15253 & 13690 & 10430 & 10372 \\
\hline \multirow{14}{*}{$\begin{array}{c}\text { Daboia russelii } \\
\text { siamensis }\end{array}$} & 17786 & 6566 & 6832 & 8152 & 4712 & 6603 & 10358 & 18853 & 14125 & 12944 & 9382 & 9256 \\
\hline & 15991 & 6326 & 7214 & 7990 & 4766 & 6778 & 9856 & 17292 & 12549 & 11945 & 9778 & 9736 \\
\hline & 16891 & 6179 & 7091 & 7523 & 4394 & 6934 & 9562 & 20896 & 12932 & 12338 & 9351 & 9545 \\
\hline & 17276 & 6193 & 6836 & 7610 & 4389 & 7290 & 8928 & 20300 & 13523 & 11413 & 9431 & 9276 \\
\hline & 17954 & 6436 & 6135 & 6690 & 4705 & 7172 & 9883 & 17294 & 12977 & 11863 & 9232 & 9429 \\
\hline & 18459 & 7358 & 5949 & 6487 & 4643 & 6911 & 9182 & 17178 & 13055 & 11586 & 9451 & 9696 \\
\hline & 20505 & 6741 & 6720 & 7531 & 4266 & 6937 & 9458 & 21567 & 13371 & 11922 & 9833 & 9214 \\
\hline & 16348 & 7049 & 5941 & 7441 & 5032 & 6986 & 9277 & 19023 & 12897 & 11337 & 10421 & 9669 \\
\hline & 19456 & 7028 & 7572 & 7126 & 4563 & 6820 & 10017 & 18055 & 15855 & 11782 & 9782 & 9523 \\
\hline & 20837 & 6589 & 7802 & 8001 & 4750 & 7503 & 9544 & 19972 & 15794 & 11301 & 9258 & 9455 \\
\hline & 20443 & 5779 & 7425 & 7674 & 4938 & 7342 & 9121 & 19107 & 14419 & 11915 & 9064 & 10715 \\
\hline & 18127 & 6881 & 6948 & 8247 & 4493 & 6922 & 9695 & 21119 & 13689 & 12031 & 9084 & 9981 \\
\hline & 16857 & 7875 & 6463 & 7461 & 5282 & 7067 & 9401 & 18224 & 14028 & 11951 & 9564 & 9562 \\
\hline & 19139 & 5919 & 6533 & 7717 & 4388 & 7026 & 10166 & 19851 & 13988 & 11822 & 9459 & 10573 \\
\hline
\end{tabular}




\begin{tabular}{|c|c|c|c|c|c|c|c|c|c|c|c|c|}
\hline \multirow{14}{*}{ Naja atra } & 16272 & 11455 & 8375 & 8052 & 5635 & 6685 & 11809 & 23572 & 10916 & 10751 & 8212 & 9399 \\
\hline & 17375 & 11826 & 8306 & 8016 & 5725 & 6575 & 11430 & 23777 & 10768 & 12392 & 8333 & 10000 \\
\hline & 16226 & 11296 & 8516 & 8440 & 6246 & 7171 & 10590 & 23506 & 12898 & 12806 & 8164 & 9632 \\
\hline & 17539 & 12927 & 8677 & 8351 & 6365 & 7272 & 11063 & 22578 & 10814 & 11342 & 8193 & 9316 \\
\hline & 18121 & 13468 & 8683 & 9674 & 5923 & 7515 & 11111 & 23298 & 11403 & 11852 & 8522 & 9650 \\
\hline & 15880 & 12296 & 9312 & 8683 & 5992 & 7516 & 10429 & 25697 & 12806 & 11498 & 8091 & 10276 \\
\hline & 16888 & 12668 & 8284 & 8662 & 5583 & 6690 & 10645 & 26569 & 12274 & 11921 & 8622 & 9338 \\
\hline & 19169 & 13009 & 9228 & 9294 & 6521 & 7609 & 11372 & 23987 & 12004 & 11999 & 8235 & 9572 \\
\hline & 16477 & 12556 & 7961 & 8732 & 6007 & 7325 & 11542 & 25830 & 12581 & 11427 & 8950 & 9669 \\
\hline & 13547 & 10220 & 8513 & 8512 & 6056 & 7463 & 10904 & 23086 & 11557 & 10846 & 8636 & 10074 \\
\hline & 16176 & 12152 & 7851 & 8759 & 5644 & 7308 & 11990 & 23227 & 12914 & 11621 & 8409 & 9695 \\
\hline & 14964 & 11182 & 8115 & 8360 & 6061 & 6741 & 11200 & 24279 & 12202 & 11073 & 8537 & 9358 \\
\hline & 16536 & 14382 & 8408 & 8718 & 6116 & 7246 & 10528 & 24770 & 11777 & 11546 & 8352 & 10475 \\
\hline & 16785 & 10061 & 8179 & 9679 & 5890 & 7232 & 10688 & 24139 & 11553 & 10879 & 8560 & 9481 \\
\hline \multirow{14}{*}{ Agkistrodon Halys } & 10992 & 10029 & 7275 & 7449 & 4863 & 6320 & 15604 & 25880 & 11245 & 10538 & 9648 & 9477 \\
\hline & 11424 & 9696 & 9132 & 7125 & 4713 & 6556 & 14338 & 23606 & 10747 & 10281 & 9408 & 9156 \\
\hline & 13923 & 12577 & 7327 & 8271 & 4977 & 6931 & 14009 & 25122 & 11057 & 10991 & 9192 & 9657 \\
\hline & 15214 & 14567 & 8392 & 8394 & 5277 & 7384 & 14230 & 25406 & 12270 & 10915 & 9748 & 9808 \\
\hline & 13600 & 11950 & 8921 & 8344 & 5004 & 7304 & 15459 & 24778 & 13336 & 10798 & 9091 & 9334 \\
\hline & 13402 & 12082 & 10789 & 8283 & 4442 & 7354 & 14420 & 23862 & 12741 & 10643 & 10024 & 9592 \\
\hline & 16586 & 14547 & 8446 & 8483 & 4327 & 6901 & 15664 & 21587 & 12546 & 11235 & 9179 & 9376 \\
\hline & 14191 & 12812 & 10038 & 8175 & 4372 & 7150 & 14228 & 23746 & 12397 & 10502 & 9862 & 9561 \\
\hline & 14035 & 12184 & 8818 & 8019 & 4507 & 6176 & 15094 & 24171 & 12594 & 10279 & 10379 & 9306 \\
\hline & 13235 & 11684 & 9292 & 8147 & 4576 & 6146 & 15138 & 24256 & 12058 & 10683 & 9691 & 9158 \\
\hline & 13379 & 10869 & 9414 & 8436 & 4170 & 6964 & 14839 & 24193 & 11460 & 10108 & 9390 & 9121 \\
\hline & 15681 & 12437 & 9620 & 7939 & 3947 & 6906 & 15515 & 23905 & 12874 & 10709 & 9438 & 9717 \\
\hline & 14381 & 12790 & 8964 & 9106 & 5128 & 6876 & 14227 & 25353 & 12354 & 11833 & 9382 & 9418 \\
\hline & 13917 & 12357 & 9361 & 7966 & 4744 & 6895 & 14789 & 26434 & 12738 & 10691 & 10203 & 9927 \\
\hline \multirow{14}{*}{$\begin{array}{c}\text { Ophiophagus } \\
\text { hannah }\end{array}$} & 20561 & 18372 & 13513 & 12155 & 5432 & 7118 & 19411 & 28452 & 13193 & 11989 & 10202 & 10007 \\
\hline & 20932 & 18760 & 13699 & 12386 & 5777 & 6910 & 19083 & 28170 & 13409 & 12209 & 9981 & 9863 \\
\hline & 21257 & 19130 & 11928 & 10843 & 6072 & 7444 & 19782 & 28867 & 11443 & 11811 & 10206 & 9957 \\
\hline & 18308 & 16257 & 12019 & 10955 & 5493 & 7081 & 20440 & 29563 & 11696 & 11132 & 10142 & 9838 \\
\hline & 20174 & 18354 & 12943 & 12489 & 5844 & 7462 & 20559 & 28762 & 13316 & 11596 & 9904 & 9783 \\
\hline & 22187 & 20195 & 13807 & 13375 & 6022 & 7589 & 19101 & 27095 & 14263 & 11856 & 9965 & 9936 \\
\hline & 22299 & 21311 & 13765 & 13154 & 5524 & 7099 & 20788 & 28958 & 14088 & 11982 & 10757 & 10091 \\
\hline & 21220 & 20404 & 13033 & 11868 & 5403 & 7047 & 19981 & 27889 & 13669 & 11650 & 10991 & 10943 \\
\hline & 21308 & 20005 & 13706 & 12262 & 5489 & 6900 & 20423 & 27015 & 14128 & 11228 & 10191 & 10557 \\
\hline & 22557 & 20343 & 14470 & 13228 & 5508 & 7234 & 20098 & 30529 & 14965 & 11086 & 10243 & 10655 \\
\hline & 22476 & 19195 & 14972 & 13439 & 5443 & 6999 & 19707 & 29555 & 14158 & 11665 & 10119 & 10940 \\
\hline & 23161 & 19245 & 13546 & 13759 & 5152 & 7537 & 20668 & 28647 & 14849 & 11569 & 10917 & 10670 \\
\hline & 21531 & 19786 & 15271 & 12834 & 5229 & 7357 & 19007 & 30460 & 14279 & 11538 & 10584 & 9928 \\
\hline & 23226 & 20526 & 15125 & 12891 & 5443 & 7171 & 20055 & 27681 & 12970 & 11616 & 10541 & 10829 \\
\hline
\end{tabular}




\begin{tabular}{|c|c|c|c|c|c|c|c|c|c|c|c|c|}
\hline \multirow{14}{*}{ Control } & 15065 & 8849 & 9344 & 12990 & 5606 & 8222 & 14267 & 21098 & 9265 & 11159 & 9698 & 9775 \\
\hline & 16381 & 8514 & 10725 & 13161 & 5836 & 8756 & 14682 & 20315 & 8629 & 10834 & 10010 & 9725 \\
\hline & 14151 & 8327 & 10461 & 12542 & 5798 & 8268 & 13386 & 21186 & 8854 & 11581 & 10063 & 10008 \\
\hline & 15527 & 8481 & 10597 & 11631 & 5625 & 7949 & 12893 & 20705 & 9749 & 11625 & 10460 & 10083 \\
\hline & 15648 & 8942 & 8959 & 12094 & 6298 & 8185 & 13083 & 20368 & 9235 & 10648 & 9609 & 9420 \\
\hline & 14252 & 8583 & 9653 & 12542 & 6185 & 7953 & 13804 & 20616 & 9559 & 11675 & 9853 & 9865 \\
\hline & 15670 & 8646 & 10670 & 12852 & 5822 & 7771 & 14777 & 21758 & 10804 & 12668 & 10722 & 10264 \\
\hline & 15412 & 8016 & 10746 & 12428 & 6523 & 8408 & 15796 & 21296 & 9414 & 11488 & 10403 & 10104 \\
\hline & 16354 & 8662 & 11653 & 13369 & 6440 & 8192 & 14588 & 22458 & 10617 & 12954 & 10893 & 10469 \\
\hline & 16395 & 9546 & 11634 & 13225 & 5535 & 8065 & 16137 & 22814 & 10327 & 13229 & 10409 & 10364 \\
\hline & 15899 & 8912 & 11721 & 12258 & 5757 & 8530 & 15651 & 22141 & 9909 & 12297 & 10254 & 10474 \\
\hline & 15820 & 8561 & 12158 & 12128 & 5559 & 8438 & 15132 & 20541 & 10793 & 11407 & 9747 & 10700 \\
\hline & 14451 & 9251 & 11762 & 12266 & 5897 & 8403 & 15160 & 21052 & 9658 & 10890 & 10374 & 10857 \\
\hline & 16688 & 8938 & 11855 & 11490 & 5331 & 8549 & 14534 & 22997 & 9640 & 10803 & 10293 & 9930 \\
\hline
\end{tabular}


Table S3 Raw fluorescence responses in six channels of the fluorescent sensor array in the discrimination of snake venoms, BSA, thrombin and TRF in human plasma.

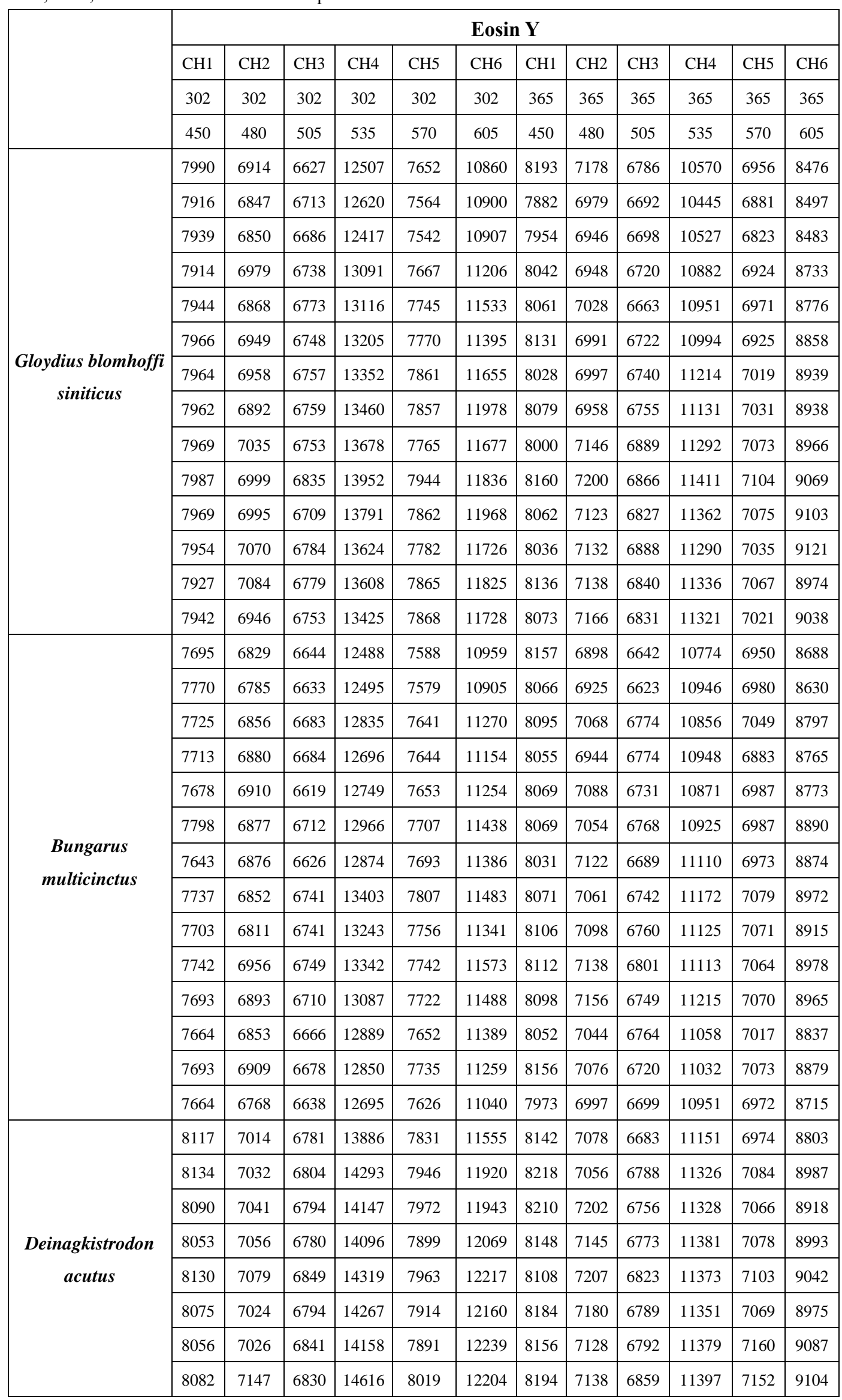




\begin{tabular}{|c|c|c|c|c|c|c|c|c|c|c|c|c|}
\hline & 8122 & 7074 & 6851 & 14630 & 7971 & 12258 & 8179 & 7205 & 6771 & 11581 & 7074 & 9219 \\
\hline & 8125 & 7096 & 6832 & 14567 & 8004 & 12349 & 8227 & 7201 & 6848 & 11535 & 7113 & 9151 \\
\hline & 8076 & 7048 & 6854 & 14151 & 7881 & 12007 & 8179 & 7241 & 6835 & 11285 & 7096 & 8933 \\
\hline & 8098 & 7114 & 6828 & 13847 & 7870 & 11847 & 8093 & 7148 & 6792 & 11191 & 7007 & 8870 \\
\hline & 8122 & 7107 & 6862 & 13914 & 7907 & 11926 & 8231 & 7224 & 6896 & 11246 & 7113 & 8979 \\
\hline & 8018 & 6978 & 6833 & 13864 & 7928 & 11987 & 8142 & 7149 & 6752 & 11275 & 7080 & 8972 \\
\hline \multirow{14}{*}{$\begin{array}{c}\text { Daboia russelii } \\
\text { siamensis }\end{array}$} & 8552 & 7187 & 6974 & 15464 & 8220 & 13035 & 8306 & 7278 & 6849 & 11232 & 7082 & 8900 \\
\hline & 8571 & 7240 & 6999 & 15752 & 8311 & 13145 & 8263 & 7401 & 6825 & 11342 & 7091 & 9031 \\
\hline & 8569 & 7274 & 7031 & 15870 & 8392 & 13426 & 8343 & 7392 & 6916 & 11409 & 7075 & 9135 \\
\hline & 8587 & 7220 & 7018 & 15803 & 8340 & 13334 & 8308 & 7272 & 6905 & 11538 & 7122 & 9035 \\
\hline & 8393 & 7220 & 7050 & 15768 & 8337 & 13371 & 8295 & 7345 & 6905 & 11446 & 7113 & 9196 \\
\hline & 8562 & 7217 & 7070 & 15673 & 8346 & 13485 & 8268 & 7341 & 6914 & 11523 & 7189 & 9191 \\
\hline & 8503 & 7215 & 7068 & 15848 & 8270 & 13609 & 8276 & 7315 & 6887 & 11538 & 7074 & 9235 \\
\hline & 8460 & 7288 & 7060 & 16322 & 8430 & 13691 & 8242 & 7315 & 6934 & 11624 & 7187 & 9133 \\
\hline & 8579 & 7252 & 7092 & 16041 & 8399 & 13689 & 8329 & 7409 & 6976 & 11605 & 7128 & 9223 \\
\hline & 8581 & 7379 & 7073 & 16069 & 8468 & 13793 & 8265 & 7435 & 6946 & 11718 & 7185 & 9261 \\
\hline & 8532 & 7222 & 7056 & 16321 & 8453 & 13910 & 8305 & 7409 & 6925 & 11678 & 7169 & 9228 \\
\hline & 8489 & 7299 & 7055 & 15880 & 8443 & 13423 & 8211 & 7359 & 6993 & 11498 & 7121 & 9219 \\
\hline & 8524 & 7334 & 6977 & 15993 & 8425 & 13606 & 8171 & 7364 & 6927 & 11535 & 7103 & 9195 \\
\hline & 8444 & 7239 & 7015 & 15668 & 8409 & 13284 & 8246 & 7342 & 6886 & 11415 & 7063 & 9104 \\
\hline \multirow{14}{*}{ Naja atra } & 9253 & 7495 & 7261 & 17139 & 8657 & 14097 & 7755 & 7298 & 6859 & 10644 & 6937 & 8488 \\
\hline & 9311 & 7512 & 7286 & 17173 & 8611 & 14181 & 7762 & 7272 & 6859 & 10829 & 6898 & 8599 \\
\hline & 9300 & 7512 & 7308 & 17262 & 8625 & 14487 & 7768 & 7342 & 6915 & 10839 & 6978 & 8665 \\
\hline & 9178 & 7481 & 7357 & 17660 & 8644 & 14455 & 7813 & 7394 & 6957 & 10857 & 6985 & 8697 \\
\hline & 9282 & 7500 & 7316 & 17713 & 8638 & 14510 & 7845 & 7365 & 6908 & 10896 & 7025 & 8665 \\
\hline & 9207 & 7435 & 7282 & 17237 & 8687 & 14361 & 7820 & 7288 & 6960 & 10870 & 6955 & 8605 \\
\hline & 9248 & 7496 & 7329 & 17532 & 8806 & 14504 & 7884 & 7470 & 6933 & 11020 & 7018 & 8769 \\
\hline & 9227 & 7628 & 7367 & 17756 & 8735 & 14800 & 7825 & 7385 & 6953 & 11020 & 7073 & 8698 \\
\hline & 9243 & 7706 & 7383 & 17677 & 8741 & 14624 & 7809 & 7416 & 6974 & 10978 & 7018 & 8687 \\
\hline & 9321 & 7713 & 7397 & 17569 & 8830 & 14715 & 7864 & 7462 & 6975 & 10912 & 7003 & 8681 \\
\hline & 9266 & 7660 & 7322 & 17575 & 8713 & 14727 & 7812 & 7471 & 6935 & 10802 & 6961 & 8591 \\
\hline & 9294 & 7679 & 7362 & 17396 & 8773 & 14566 & 7935 & 7518 & 7061 & 10733 & 7006 & 8596 \\
\hline & 9070 & 7452 & 7258 & 17328 & 8698 & 14481 & 7692 & 7370 & 6907 & 10742 & 6983 & 8536 \\
\hline & 9053 & 7538 & 7243 & 16841 & 8636 & 14172 & 7725 & 7314 & 6888 & 10583 & 6958 & 8447 \\
\hline \multirow{8}{*}{ Agkistrodon Halys } & 9605 & 7741 & 7379 & 17972 & 8853 & 14757 & 7688 & 7366 & 6941 & 10728 & 6975 & 8539 \\
\hline & 9458 & 7626 & 7326 & 18559 & 8854 & 15267 & 7622 & 7210 & 6841 & 10783 & 6939 & 8630 \\
\hline & 9523 & 7678 & 7399 & 18725 & 8970 & 15508 & 7681 & 7367 & 6917 & 10968 & 6971 & 8786 \\
\hline & 9529 & 7725 & 7370 & 19025 & 9043 & 15495 & 7703 & 7378 & 6982 & 11064 & 7017 & 8799 \\
\hline & 9495 & 7642 & 7309 & 19066 & 9020 & 15734 & 7629 & 7349 & 6900 & 11136 & 7007 & 8720 \\
\hline & 9455 & 7698 & 7390 & 18653 & 9037 & 15680 & 7651 & 7382 & 6850 & 11048 & 7024 & 8722 \\
\hline & 9482 & 7753 & 7408 & 19060 & 9107 & 15766 & 7678 & 7374 & 6929 & 11229 & 7067 & 8820 \\
\hline & 9555 & 7744 & 7393 & 19515 & 9203 & 16156 & 7706 & 7368 & 6911 & 11199 & 7106 & 8826 \\
\hline
\end{tabular}




\begin{tabular}{|c|c|c|c|c|c|c|c|c|c|c|c|c|}
\hline & 9610 & 7762 & 7468 & 19515 & 9199 & 16243 & 7721 & 7392 & 6987 & 11236 & 7087 & 8859 \\
\hline & 9564 & 7806 & 7413 & 19441 & 9130 & 16101 & 7750 & 7422 & 6937 & 11130 & 7087 & 8827 \\
\hline & 9418 & 7742 & 7344 & 19005 & 9104 & 15892 & 7715 & 7395 & 6935 & 11003 & 7025 & 8673 \\
\hline & 9388 & 7741 & 7362 & 17967 & 8891 & 15140 & 7650 & 7431 & 6948 & 10826 & 6947 & 8563 \\
\hline & 9347 & 7584 & 7246 & 18804 & 9080 & 15628 & 7657 & 7324 & 6843 & 10781 & 7006 & 8598 \\
\hline & 9245 & 7584 & 7303 & 18375 & 8977 & 15400 & 7552 & 7218 & 6834 & 10649 & 6993 & 8621 \\
\hline \multirow{14}{*}{$\begin{array}{c}\text { Ophiophagus } \\
\text { hannah }\end{array}$} & 9140 & 7545 & 7281 & 17373 & 8681 & 14578 & 8083 & 7440 & 7016 & 11618 & 7223 & 9354 \\
\hline & 9154 & 7599 & 7222 & 17632 & 8855 & 14723 & 8182 & 7456 & 7009 & 11737 & 7253 & 9531 \\
\hline & 9198 & 7543 & 7229 & 17889 & 8849 & 15142 & 8206 & 7504 & 7058 & 11769 & 7234 & 9473 \\
\hline & 9171 & 7587 & 7282 & 17755 & 8773 & 15166 & 8176 & 7551 & 7016 & 11887 & 7305 & 9571 \\
\hline & 9340 & 7615 & 7230 & 18134 & 8863 & 15132 & 8224 & 7523 & 7036 & 12020 & 7349 & 9601 \\
\hline & 9141 & 7590 & 7294 & 18387 & 8903 & 15436 & 8241 & 7556 & 7029 & 12093 & 7300 & 9645 \\
\hline & 9129 & 7619 & 7240 & 17967 & 8920 & 15402 & 8246 & 7586 & 7060 & 12144 & 7360 & 9728 \\
\hline & 9298 & 7632 & 7323 & 18293 & 8935 & 15534 & 8305 & 7607 & 7088 & 12283 & 7354 & 9690 \\
\hline & 9230 & 7642 & 7365 & 18503 & 9015 & 15657 & 8249 & 7621 & 7171 & 12298 & 7431 & 9817 \\
\hline & 9222 & 7673 & 7346 & 18288 & 8984 & 15489 & 8341 & 7692 & 7106 & 12196 & 7380 & 9699 \\
\hline & 9233 & 7662 & 7325 & 18469 & 8995 & 15654 & 8301 & 7647 & 7135 & 12152 & 7410 & 9754 \\
\hline & 9136 & 7634 & 7296 & 18080 & 8975 & 15426 & 8197 & 7605 & 7076 & 12045 & 7337 & 9733 \\
\hline & 9051 & 7585 & 7254 & 17979 & 8966 & 15266 & 8145 & 7604 & 7081 & 12014 & 7336 & 9623 \\
\hline & 9031 & 7542 & 7228 & 17554 & 8855 & 14988 & 8082 & 7559 & 7113 & 11815 & 7278 & 9455 \\
\hline \multirow{14}{*}{ BSA } & 7786 & 6996 & 6697 & 13254 & 8342 & 13209 & 8010 & 7127 & 6735 & 10522 & 7153 & 9083 \\
\hline & 7938 & 7039 & 6727 & 13875 & 8463 & 13661 & 8111 & 7138 & 6837 & 10548 & 7136 & 9107 \\
\hline & 8390 & 7243 & 6815 & 15924 & 9184 & 15970 & 8216 & 7408 & 6933 & 10732 & 7214 & 9294 \\
\hline & 8966 & 7511 & 7063 & 18673 & 9920 & 18469 & 7712 & 7333 & 6885 & 10330 & 7104 & 8932 \\
\hline & 9226 & 7675 & 7239 & 19490 & 10312 & 19353 & 7594 & 7356 & 6907 & 10313 & 7069 & 8830 \\
\hline & 8863 & 7498 & 7067 & 17975 & 9822 & 17993 & 8029 & 7573 & 6974 & 10911 & 7316 & 9535 \\
\hline & 8386 & 7361 & 6986 & 16410 & 9352 & 16433 & 8237 & 7501 & 6978 & 11160 & 7433 & 9728 \\
\hline & 7721 & 6867 & 6657 & 12883 & 8276 & 13010 & 7914 & 7062 & 6779 & 10454 & 7152 & 9010 \\
\hline & 7907 & 6891 & 6616 & 13708 & 8372 & 13625 & 7964 & 7145 & 6720 & 10491 & 7093 & 8953 \\
\hline & 8352 & 7177 & 6830 & 15884 & 9114 & 15753 & 8141 & 7337 & 6866 & 10649 & 7238 & 9260 \\
\hline & 9054 & 7428 & 7028 & 18555 & 9920 & 18274 & 7604 & 7279 & 6826 & 10233 & 7065 & 8811 \\
\hline & 9087 & 7573 & 7086 & 19236 & 10073 & 19035 & 7464 & 7315 & 6842 & 10108 & 6983 & 8714 \\
\hline & 8889 & 7515 & 6940 & 17855 & 9637 & 17743 & 7953 & 7388 & 6859 & 10834 & 7275 & 9357 \\
\hline & 8408 & 7323 & 6879 & 16178 & 9253 & 16089 & 8178 & 7438 & 6879 & 11114 & 7277 & 9517 \\
\hline \multirow{8}{*}{ Thrombin } & 8394 & 8070 & 7072 & 20853 & 12057 & 24016 & 9058 & 9210 & 9292 & 16849 & 8364 & 9752 \\
\hline & 7653 & 8174 & 7470 & 22883 & 10084 & 20847 & 7811 & 10463 & 9130 & 17041 & 8418 & 10675 \\
\hline & 7822 & 8012 & 7488 & 22098 & 11431 & 19637 & 8171 & 9915 & 8801 & 18992 & 8384 & 9754 \\
\hline & 8019 & 8891 & 8069 & 24002 & 11501 & 20413 & 8614 & 10379 & 8391 & 17327 & 8448 & 10046 \\
\hline & 7970 & 9339 & 7380 & 20573 & 11872 & 20033 & 9066 & 8772 & 8716 & 17225 & 8636 & 10935 \\
\hline & 8517 & 8362 & 8040 & 19815 & 12284 & 21087 & 9396 & 9340 & 9014 & 18140 & 8982 & 10885 \\
\hline & 8868 & 7654 & 7968 & 19347 & 11485 & 21384 & 9593 & 9492 & 9451 & 17825 & 8841 & 10809 \\
\hline & 8626 & 8321 & 7337 & 24185 & 11266 & 21175 & 9849 & 9535 & 8939 & 16720 & 8129 & 10323 \\
\hline
\end{tabular}




\begin{tabular}{|c|c|c|c|c|c|c|c|c|c|c|c|c|}
\hline & 9215 & 8550 & 7501 & 21125 & 11315 & 20901 & 9429 & 10283 & 9314 & 16940 & 8112 & 9360 \\
\hline & 8697 & 8943 & 7512 & 20667 & 10755 & 22976 & 8521 & 10239 & 9444 & 16393 & 9001 & 9625 \\
\hline & 7938 & 8362 & 7945 & 20698 & 11655 & 23633 & 8037 & 8920 & 8918 & 17439 & 8471 & 9922 \\
\hline & 8571 & 7769 & 7072 & 20509 & 12397 & 19326 & 8147 & 8940 & 9312 & 17905 & 9145 & 9446 \\
\hline & 7916 & 8435 & 8082 & 20923 & 12321 & 20156 & 8278 & 9453 & 9054 & 18983 & 8669 & 9804 \\
\hline & 8639 & 8110 & 8128 & 21099 & 12590 & 20472 & 9219 & 9421 & 9741 & 17355 & 8439 & 10091 \\
\hline \multirow{14}{*}{ TRF } & 7580 & 6745 & 6665 & 12082 & 7510 & 10533 & 7783 & 6951 & 6656 & 10543 & 6949 & 8407 \\
\hline & 7639 & 6787 & 6604 & 12521 & 7552 & 10835 & 7879 & 6933 & 6724 & 10684 & 6936 & 8392 \\
\hline & 8140 & 7044 & 6815 & 14547 & 8032 & 12260 & 7796 & 7062 & 6704 & 10820 & 6969 & 8766 \\
\hline & 8695 & 7250 & 7034 & 16754 & 8589 & 13926 & 7340 & 7123 & 6744 & 10418 & 6900 & 8317 \\
\hline & 8765 & 7286 & 7065 & 17107 & 8682 & 14176 & 7296 & 7102 & 6717 & 10358 & 6831 & 8250 \\
\hline & 8533 & 7202 & 6914 & 16118 & 8414 & 13606 & 7737 & 7159 & 6796 & 11057 & 7063 & 8829 \\
\hline & 8066 & 7058 & 6784 & 14828 & 8135 & 12710 & 8031 & 7246 & 6732 & 11242 & 7167 & 8949 \\
\hline & 7652 & 6827 & 6591 & 12090 & 7567 & 10614 & 7825 & 6977 & 6676 & 10579 & 6948 & 8583 \\
\hline & 7761 & 6853 & 6602 & 13092 & 7717 & 11193 & 8006 & 7017 & 6706 & 10894 & 6955 & 8648 \\
\hline & 8111 & 7078 & 6855 & 14825 & 8168 & 12541 & 7953 & 7183 & 6742 & 11057 & 7045 & 8732 \\
\hline & 8744 & 7228 & 6973 & 16808 & 8682 & 14017 & 7469 & 7193 & 6741 & 10368 & 6977 & 8447 \\
\hline & 8928 & 7352 & 7072 & 17558 & 8841 & 14493 & 7421 & 7174 & 6746 & 10455 & 6979 & 8246 \\
\hline & 8652 & 7235 & 7085 & 16199 & 8546 & 13766 & 7809 & 7290 & 6821 & 11147 & 7078 & 8905 \\
\hline & 8230 & 7127 & 6829 & 14881 & 8316 & 12775 & 8034 & 7319 & 6796 & 11408 & 7146 & 9057 \\
\hline \multirow{14}{*}{ Control } & 8516 & 7238 & 6975 & 17503 & 8617 & 14560 & 8356 & 7354 & 6859 & 11972 & 7244 & 9455 \\
\hline & 8690 & 7356 & 7067 & 17494 & 8659 & 14733 & 8405 & 7381 & 6911 & 12038 & 7282 & 9561 \\
\hline & 8748 & 7366 & 7113 & 17835 & 8746 & 14889 & 8414 & 7417 & 6912 & 12277 & 7303 & 9608 \\
\hline & 8559 & 7278 & 7054 & 17682 & 8687 & 14688 & 8440 & 7332 & 6872 & 12248 & 7280 & 9557 \\
\hline & 8716 & 7383 & 7114 & 18335 & 8914 & 15231 & 8457 & 7444 & 6898 & 12397 & 7347 & 9758 \\
\hline & 8655 & 7281 & 7134 & 17919 & 8816 & 15009 & 8369 & 7342 & 6876 & 12459 & 7257 & 9732 \\
\hline & 8684 & 7322 & 7175 & 18350 & 8870 & 15170 & 8464 & 7561 & 6950 & 12532 & 7348 & 9812 \\
\hline & 8687 & 7412 & 7082 & 18305 & 8854 & 15253 & 8449 & 7483 & 6919 & 12542 & 7294 & 9818 \\
\hline & 8674 & 7362 & 7202 & 18089 & 8760 & 15035 & 8449 & 7485 & 6946 & 12456 & 7376 & 9772 \\
\hline & 8568 & 7414 & 7146 & 17893 & 8778 & 14836 & 8385 & 7453 & 6943 & 12369 & 7345 & 9710 \\
\hline & 8552 & 7433 & 7097 & 17909 & 8792 & 14881 & 8382 & 7485 & 6895 & 12362 & 7352 & 9711 \\
\hline & 8496 & 7309 & 7101 & 17388 & 8685 & 14492 & 8335 & 7395 & 6960 & 12103 & 7252 & 9589 \\
\hline & 8383 & 7306 & 7008 & 17103 & 8684 & 14326 & 8260 & 7351 & 6902 & 11945 & 7267 & 9450 \\
\hline & 8389 & 7291 & 6988 & 17002 & 8610 & 14265 & 8244 & 7464 & 6878 & 11955 & 7226 & 9432 \\
\hline \multirow{7}{*}{$\begin{array}{c}\text { Gloydius blomhoffi } \\
\text { siniticus }\end{array}$} & \multicolumn{12}{|c|}{ FITC } \\
\hline & CH1 & $\mathrm{CH} 2$ & $\mathrm{CH} 3$ & $\mathrm{CH} 4$ & CH5 & CH6 & $\mathrm{CH} 1$ & $\mathrm{CH} 2$ & $\mathrm{CH} 3$ & $\mathrm{CH} 4$ & CH5 & CH6 \\
\hline & 302 & 302 & 302 & 302 & 302 & 302 & 365 & 365 & 365 & 365 & 365 & 365 \\
\hline & 450 & 480 & 505 & 535 & 570 & 605 & 450 & 480 & 505 & 535 & 570 & 605 \\
\hline & 8535 & 7274 & 8134 & 10100 & 6952 & 8702 & 8416 & 7358 & 7887 & 11016 & 6760 & 8175 \\
\hline & 8416 & 7326 & 8191 & 10065 & 6950 & 8782 & 8442 & 7431 & 7826 & 11128 & 6834 & 8250 \\
\hline & 8405 & 7364 & 8278 & 10253 & 7007 & 8844 & 8545 & 7419 & 7989 & 11129 & 6866 & 8365 \\
\hline
\end{tabular}




\begin{tabular}{|c|c|c|c|c|c|c|c|c|c|c|c|c|}
\hline & 8506 & 7396 & 8364 & 10372 & 6938 & 8971 & 8505 & 7502 & 8081 & 11373 & 6908 & 8315 \\
\hline & 8540 & 7494 & 8393 & 10472 & 6949 & 9003 & 8571 & 7529 & 8105 & 11489 & 6911 & 8449 \\
\hline & 8595 & 7529 & 8315 & 10481 & 7005 & 9038 & 8604 & 7563 & 8165 & 11554 & 6949 & 8492 \\
\hline & 8498 & 7431 & 8487 & 10610 & 7002 & 9136 & 8590 & 7572 & 8130 & 11770 & 7000 & 8572 \\
\hline & 8542 & 7475 & 8436 & 10806 & 7011 & 9167 & 8542 & 7581 & 8178 & 11790 & 6995 & 8568 \\
\hline & 8591 & 7583 & 8400 & 10838 & 7116 & 9180 & 8640 & 7665 & 8292 & 11724 & 7043 & 8675 \\
\hline & 8534 & 7464 & 8468 & 10977 & 7097 & 9106 & 8600 & 7590 & 8246 & 11737 & 6976 & 8679 \\
\hline & 8538 & 7569 & 8431 & 10843 & 7071 & 9093 & 8597 & 7682 & 8238 & 11773 & 7030 & 8649 \\
\hline & 8486 & 7433 & 8388 & 10741 & 7006 & 9056 & 8546 & 7576 & 8210 & 11703 & 7025 & 8580 \\
\hline & 8463 & 7462 & 8248 & 10471 & 6997 & 8950 & 8455 & 7584 & 8045 & 11514 & 6908 & 8439 \\
\hline & 8291 & 7361 & 8199 & 10269 & 7005 & 8774 & 8328 & 7516 & 7979 & 11252 & 6892 & 8325 \\
\hline \multirow{14}{*}{$\begin{array}{c}\text { Bungarus } \\
\text { multicinctus }\end{array}$} & 8501 & 7471 & 8228 & 10372 & 7005 & 8990 & 8722 & 7508 & 8018 & 11365 & 6899 & 8379 \\
\hline & 8469 & 7459 & 8204 & 10671 & 7057 & 8960 & 8713 & 7564 & 7978 & 11584 & 6877 & 8316 \\
\hline & 8533 & 7528 & 8326 & 10614 & 7008 & 9101 & 8647 & 7533 & 8171 & 11699 & 6944 & 8478 \\
\hline & 8552 & 7574 & 8338 & 10631 & 7076 & 9149 & 8713 & 7632 & 8230 & 11790 & 6975 & 8607 \\
\hline & 8613 & 7510 & 8304 & 10721 & 7082 & 9176 & 8666 & 7655 & 8143 & 11844 & 6975 & 8606 \\
\hline & 8472 & 7536 & 8444 & 10903 & 7124 & 9227 & 8728 & 7646 & 8248 & 12015 & 6974 & 8732 \\
\hline & 8649 & 7603 & 8494 & 11038 & 7165 & 9280 & 8765 & 7747 & 8305 & 12014 & 7042 & 8764 \\
\hline & 8488 & 7609 & 8415 & 10962 & 7065 & 9259 & 8752 & 7717 & 8269 & 11958 & 7063 & 8747 \\
\hline & 8441 & 7622 & 8396 & 10966 & 7068 & 9290 & 8666 & 7774 & 8324 & 11894 & 7034 & 8784 \\
\hline & 8475 & 7570 & 8310 & 10684 & 7041 & 9127 & 8659 & 7694 & 8189 & 11706 & 7038 & 8635 \\
\hline & 8418 & 7488 & 8273 & 10625 & 7026 & 9024 & 8618 & 7733 & 8144 & 11598 & 7030 & 8592 \\
\hline & 8355 & 7498 & 8252 & 10524 & 7044 & 9018 & 8542 & 7641 & 8082 & 11549 & 6961 & 8550 \\
\hline & 8159 & 7412 & 8160 & 10495 & 6962 & 8901 & 8384 & 7530 & 7977 & 11453 & 6890 & 8490 \\
\hline & 8144 & 7351 & 8080 & 10431 & 6952 & 8831 & 8310 & 7481 & 7996 & 11301 & 6930 & 8390 \\
\hline \multirow{14}{*}{$\begin{array}{c}\text { Deinagkistrodon } \\
\text { acutus }\end{array}$} & 9107 & 7480 & 8315 & 11082 & 7111 & 9427 & 8452 & 7421 & 7905 & 11230 & 6860 & 8338 \\
\hline & 9019 & 7575 & 8452 & 11509 & 7125 & 9263 & 8413 & 7451 & 7963 & 11371 & 6913 & 8433 \\
\hline & 9008 & 7618 & 8666 & 11710 & 7265 & 9630 & 8541 & 7602 & 8100 & 11549 & 6937 & 8555 \\
\hline & 9081 & 7619 & 8649 & 11696 & 7228 & 9704 & 8499 & 7637 & 8066 & 11632 & 6956 & 8551 \\
\hline & 9106 & 7664 & 8699 & 11601 & 7197 & 9727 & 8523 & 7679 & 8126 & 11762 & 6986 & 8622 \\
\hline & 9157 & 7738 & 8616 & 11731 & 7243 & 9779 & 8553 & 7684 & 8218 & 11827 & 7056 & 8749 \\
\hline & 9141 & 7720 & 8754 & 12040 & 7305 & 9888 & 8569 & 7782 & 8279 & 12049 & 7001 & 8745 \\
\hline & 9170 & 7788 & 8858 & 11983 & 7278 & 9900 & 8670 & 7805 & 8298 & 12082 & 7102 & 8894 \\
\hline & 9192 & 7748 & 8833 & 12057 & 7298 & 9901 & 8606 & 7802 & 8218 & 12068 & 7101 & 8861 \\
\hline & 9210 & 7812 & 8841 & 12085 & 7233 & 9967 & 8707 & 7786 & 8328 & 12138 & 7047 & 8890 \\
\hline & 9191 & 7786 & 8867 & 12064 & 7358 & 9909 & 8617 & 7821 & 8326 & 12038 & 7122 & 8875 \\
\hline & 9072 & 7771 & 8707 & 11882 & 7282 & 9852 & 8560 & 7776 & 8305 & 11928 & 7100 & 8814 \\
\hline & 9028 & 7705 & 8654 & 11862 & 7293 & 9777 & 8516 & 7771 & 8194 & 11872 & 7028 & 8775 \\
\hline & 9082 & 7660 & 8614 & 11615 & 7234 & 9645 & 8697 & 7855 & 8195 & 11729 & 7019 & 8711 \\
\hline \multirow{2}{*}{$\begin{array}{c}\text { Daboia russelii } \\
\text { siamensis }\end{array}$} & 9653 & 7811 & 8692 & 11984 & 7342 & 10026 & 7786 & 7503 & 7670 & 10849 & 6837 & 8081 \\
\hline & 9578 & 7798 & 8746 & 12178 & 7259 & 9971 & 7899 & 7491 & 7714 & 11007 & 6814 & 8144 \\
\hline
\end{tabular}




\begin{tabular}{|c|c|c|c|c|c|c|c|c|c|c|c|c|}
\hline & 9620 & 7893 & 8992 & 12574 & 7330 & 9971 & 7961 & 7530 & 7824 & 11046 & 6856 & 8280 \\
\hline & 9601 & 7912 & 9000 & 12552 & 7392 & 10259 & 7898 & 7584 & 7892 & 11232 & 6901 & 8319 \\
\hline & 9720 & 7966 & 9064 & 12672 & 7409 & 10289 & 7971 & 7659 & 7900 & 11284 & 6904 & 8306 \\
\hline & 9735 & 8014 & 9079 & 12884 & 7487 & 10383 & 7967 & 7712 & 8006 & 11417 & 6966 & 8422 \\
\hline & 9755 & 7962 & 9123 & 13084 & 7520 & 10440 & 7994 & 7741 & 8026 & 11590 & 7029 & 8510 \\
\hline & 9750 & 7966 & 9161 & 12930 & 7469 & 10503 & 8082 & 7742 & 8074 & 11602 & 6987 & 8548 \\
\hline & 9665 & 7989 & 9099 & 12897 & 7479 & 10429 & 8123 & 7776 & 8004 & 11587 & 6907 & 8566 \\
\hline & 9649 & 7951 & 9073 & 12800 & 7430 & 10431 & 8040 & 7739 & 7994 & 11493 & 6909 & 8491 \\
\hline & 9639 & 7968 & 9023 & 12653 & 7404 & 10325 & 8056 & 7765 & 8001 & 11345 & 6913 & 8476 \\
\hline & 9444 & 7899 & 8950 & 12539 & 7406 & 10150 & 7943 & 7689 & 7903 & 11310 & 6926 & 8423 \\
\hline & 9509 & 7857 & 8966 & 12675 & 7423 & 10264 & 7868 & 7722 & 8014 & 11353 & 6953 & 8452 \\
\hline & 9353 & 7835 & 8800 & 12374 & 7362 & 10072 & 7917 & 7643 & 7942 & 11111 & 6971 & 8371 \\
\hline \multirow{14}{*}{ Naja atra } & 9664 & 7785 & 8022 & 10114 & 6986 & 8796 & 7601 & 7396 & 7325 & 9826 & 6612 & 7426 \\
\hline & 9700 & 7759 & 8122 & 10263 & 7011 & 8944 & 7612 & 7444 & 7408 & 9936 & 6683 & 7561 \\
\hline & 9610 & 7847 & 8264 & 10496 & 7045 & 8912 & 7715 & 7526 & 7430 & 10061 & 6683 & 7693 \\
\hline & 9747 & 7898 & 8321 & 10440 & 7079 & 8991 & 7820 & 7612 & 7587 & 10187 & 6660 & 7717 \\
\hline & 9821 & 7922 & 8313 & 10640 & 7074 & 9063 & 7863 & 7617 & 7610 & 10218 & 6766 & 7773 \\
\hline & 9852 & 8001 & 8195 & 10669 & 7139 & 9046 & 7912 & 7676 & 7507 & 10373 & 6747 & 7823 \\
\hline & 9800 & 7985 & 8368 & 10742 & 7167 & 9286 & 7922 & 7676 & 7678 & 10401 & 6786 & 7930 \\
\hline & 9725 & 7953 & 8331 & 10744 & 7134 & 9115 & 7873 & 7683 & 7592 & 10432 & 6799 & 7858 \\
\hline & 9751 & 7968 & 8431 & 10819 & 7169 & 9240 & 7861 & 7729 & 7677 & 10443 & 6757 & 7884 \\
\hline & 9753 & 8027 & 8377 & 10834 & 7164 & 9170 & 8000 & 7729 & 7618 & 10428 & 6817 & 7938 \\
\hline & 9675 & 7925 & 8327 & 10656 & 7102 & 9132 & 7868 & 7707 & 7611 & 10417 & 6777 & 7888 \\
\hline & 9495 & 7929 & 8249 & 10571 & 7104 & 9090 & 7759 & 7633 & 7612 & 10241 & 6749 & 7804 \\
\hline & 9478 & 7884 & 8321 & 10568 & 7074 & 8997 & 7769 & 7661 & 7608 & 10141 & 6736 & 7775 \\
\hline & 9299 & 7791 & 8135 & 10357 & 7051 & 8986 & 7757 & 7571 & 7543 & 10065 & 6733 & 7742 \\
\hline \multirow{15}{*}{ Agkistrodon Halys } & 9223 & 7510 & 8522 & 11313 & 7079 & 9507 & 8155 & 7435 & 7809 & 11086 & 6850 & 8186 \\
\hline & 9214 & 7608 & 8480 & 11726 & 7196 & 9645 & 8215 & 7557 & 7898 & 11241 & 6882 & 8285 \\
\hline & 9208 & 7619 & 8599 & 11728 & 7203 & 9618 & 8311 & 7592 & 8003 & 11283 & 6928 & 8416 \\
\hline & 9217 & 7684 & 8586 & 11822 & 7205 & 9714 & 8393 & 7534 & 8034 & 11467 & 6925 & 8441 \\
\hline & 9228 & 7618 & 8696 & 11913 & 7227 & 9766 & 8313 & 7620 & 8059 & 11491 & 7012 & 8521 \\
\hline & 9259 & 7664 & 8749 & 12043 & 7272 & 9738 & 8407 & 7647 & 8037 & 11631 & 6964 & 8632 \\
\hline & 9176 & 7640 & 8675 & 11873 & 7265 & 9770 & 8314 & 7624 & 8045 & 11667 & 7029 & 8596 \\
\hline & 9180 & 7701 & 8722 & 11998 & 7269 & 9813 & 8338 & 7663 & 8070 & 11697 & 7027 & 8690 \\
\hline & 9181 & 7716 & 8765 & 12130 & 7334 & 9930 & 8377 & 7716 & 8160 & 11790 & 7068 & 8761 \\
\hline & 9124 & 7759 & 8763 & 12112 & 7288 & 9908 & 8371 & 7687 & 8136 & 11809 & 6953 & 8697 \\
\hline & 9178 & 7706 & 8710 & 12137 & 7300 & 9864 & 8330 & 7727 & 8145 & 11747 & 7038 & 8780 \\
\hline & 9053 & 7689 & 8635 & 11881 & 7306 & 9748 & 8235 & 7660 & 8073 & 11563 & 7038 & 8659 \\
\hline & 9009 & 7604 & 8603 & 11772 & 7189 & 9693 & 8214 & 7662 & 8108 & 11512 & 7004 & 8594 \\
\hline & 8953 & 7545 & 8568 & 11640 & 7273 & 9604 & 8176 & 7601 & 7989 & 11400 & 6999 & 8551 \\
\hline & 8587 & 7258 & 7891 & 10269 & 6931 & 8630 & 8522 & 7357 & 7840 & 11165 & 6955 & 8331 \\
\hline
\end{tabular}




\begin{tabular}{|c|c|c|c|c|c|c|c|c|c|c|c|c|}
\hline \multirow{13}{*}{$\begin{array}{c}\text { Ophiophagus } \\
\text { hannah }\end{array}$} & 8472 & 7336 & 7943 & 10221 & 6904 & 8780 & 8517 & 7458 & 7850 & 11184 & 6914 & 8449 \\
\hline & 8547 & 7382 & 8083 & 10311 & 6966 & 8871 & 8512 & 7508 & 7986 & 11249 & 6927 & 8498 \\
\hline & 8565 & 7373 & 7986 & 10447 & 6926 & 8888 & 8662 & 7584 & 7890 & 11393 & 6958 & 8519 \\
\hline & 8577 & 7445 & 8063 & 10572 & 6903 & 8852 & 8555 & 7612 & 7953 & 11510 & 6967 & 8625 \\
\hline & 8644 & 7499 & 8078 & 10584 & 7067 & 8945 & 8646 & 7645 & 8002 & 11611 & 6963 & 8642 \\
\hline & 8566 & 7405 & 8132 & 10719 & 7026 & 9005 & 8580 & 7680 & 8040 & 11655 & 7048 & 8636 \\
\hline & 8537 & 7421 & 8100 & 10510 & 7028 & 8889 & 8570 & 7699 & 8015 & 11496 & 6992 & 8691 \\
\hline & 8587 & 7416 & 8111 & 10755 & 7075 & 9095 & 8553 & 7557 & 8041 & 11692 & 7016 & 8675 \\
\hline & 8556 & 7409 & 8106 & 10688 & 6989 & 9019 & 8553 & 7640 & 8041 & 11688 & 7036 & 8733 \\
\hline & 8529 & 7376 & 8012 & 10545 & 7001 & 8913 & 8517 & 7619 & 7993 & 11549 & 7031 & 8695 \\
\hline & 8509 & 7315 & 7966 & 10386 & 6997 & 8847 & 8547 & 7610 & 7971 & 11442 & 6934 & 8568 \\
\hline & 8334 & 7316 & 7946 & 10373 & 6896 & 8910 & 8451 & 7551 & 7996 & 11526 & 6992 & 8613 \\
\hline & 8402 & 7276 & 7979 & 10410 & 7021 & 8788 & 8419 & 7597 & 8049 & 11407 & 6968 & 8612 \\
\hline \multirow{14}{*}{ BSA } & 8182 & 7297 & 7602 & 9161 & 6644 & 7858 & 8382 & 7487 & 7696 & 10905 & 6743 & 7968 \\
\hline & 8248 & 7305 & 7610 & 9560 & 6771 & 8179 & 8428 & 7501 & 7767 & 11028 & 6814 & 8026 \\
\hline & 8793 & 7499 & 7762 & 9985 & 6819 & 8439 & 8352 & 7623 & 7729 & 11007 & 6846 & 8158 \\
\hline & 8832 & 7498 & 7777 & 10030 & 6870 & 8504 & 8141 & 7553 & 7741 & 10909 & 6822 & 8059 \\
\hline & 8236 & 7178 & 7498 & 9389 & 6758 & 8010 & 8315 & 7388 & 7681 & 10851 & 6742 & 7921 \\
\hline & 8075 & 7179 & 7274 & 9133 & 6732 & 7917 & 8422 & 7377 & 7651 & 10759 & 6815 & 7964 \\
\hline & 8153 & 7244 & 7435 & 9094 & 6607 & 7732 & 8174 & 7362 & 7636 & 10718 & 6734 & 7876 \\
\hline & 8096 & 7276 & 7489 & 9264 & 6686 & 8005 & 8326 & 7421 & 7700 & 10868 & 6735 & 7889 \\
\hline & 8612 & 7386 & 7606 & 9891 & 6811 & 8277 & 8250 & 7542 & 7695 & 10925 & 6816 & 8072 \\
\hline & 9176 & 7627 & 7959 & 10520 & 6892 & 8747 & 7594 & 7442 & 7544 & 10409 & 6692 & 7775 \\
\hline & 9130 & 7632 & 8047 & 10567 & 6969 & 8774 & 7589 & 7408 & 7479 & 10328 & 6691 & 7778 \\
\hline & 8739 & 7374 & 7659 & 9797 & 6783 & 8330 & 8048 & 7452 & 7679 & 10701 & 6683 & 7917 \\
\hline & 8177 & 7102 & 7328 & 9243 & 6736 & 7899 & 8149 & 7250 & 7535 & 10661 & 6718 & 7859 \\
\hline & 8044 & 7089 & 7328 & 8968 & 6682 & 7814 & 8435 & 7261 & 7544 & 10755 & 6651 & 7920 \\
\hline \multirow{14}{*}{ Thrombin } & 9882 & 6546 & 10339 & 15491 & 7978 & 10599 & 8861 & 6137 & 7750 & 10480 & 7509 & 12105 \\
\hline & 9434 & 6541 & 10198 & 16330 & 7894 & 10064 & 8687 & 5188 & 9941 & 10987 & 7738 & 12370 \\
\hline & 9717 & 7091 & 10272 & 14711 & 8200 & 10571 & 9096 & 6186 & 9731 & 10358 & 7508 & 13294 \\
\hline & 9633 & 7293 & 10412 & 14734 & 8096 & 10587 & 9720 & 5861 & 8283 & 11190 & 7021 & 11966 \\
\hline & 8966 & 7521 & 10300 & 14089 & 7923 & 10405 & 10233 & 5331 & 9203 & 11345 & 7091 & 12273 \\
\hline & 10770 & 7206 & 10011 & 14368 & 8301 & 10117 & 10375 & 5331 & 8170 & 11466 & 7164 & 13032 \\
\hline & 9569 & 6793 & 10028 & 14196 & 8880 & 11036 & 10689 & 5858 & 7755 & 12025 & 7694 & 12948 \\
\hline & 8744 & 7238 & 11555 & 15771 & 8908 & 10290 & 10319 & 6330 & 8326 & 12947 & 7171 & 12211 \\
\hline & 9169 & 6787 & 11737 & 15303 & 9147 & 10954 & 10548 & 5579 & 8266 & 11473 & 7046 & 12730 \\
\hline & 10106 & 6963 & 11804 & 18156 & 9119 & 11097 & 10309 & 5729 & 8833 & 10757 & 7020 & 12289 \\
\hline & 10453 & 7051 & 13177 & 17543 & 8201 & 11638 & 10036 & 5548 & 8768 & 13073 & 7353 & 12727 \\
\hline & 10033 & 7249 & 13376 & 15930 & 8995 & 10999 & 9566 & 5841 & 8720 & 11294 & 7503 & 12143 \\
\hline & 9836 & 7485 & 10470 & 16741 & 9366 & 10517 & 9443 & 5144 & 7932 & 10996 & 7538 & 13291 \\
\hline & 9522 & 7210 & 11858 & 15685 & 8928 & 11074 & 10233 & 5079 & 8123 & 10610 & 7839 & 12102 \\
\hline TRF & 7946 & 7149 & 7944 & 10057 & 6899 & 8530 & 8082 & 7222 & 7650 & 10908 & 6840 & 8096 \\
\hline
\end{tabular}




\begin{tabular}{|c|c|c|c|c|c|c|c|c|c|c|c|c|}
\hline & 7958 & 7144 & 7938 & 10453 & 6977 & 8818 & 8170 & 7328 & 7698 & 10967 & 6881 & 8210 \\
\hline & 8382 & 7312 & 8203 & 11073 & 7110 & 9110 & 8048 & 7415 & 7770 & 11058 & 6956 & 8271 \\
\hline & 8697 & 7394 & 8452 & 11895 & 7286 & 9608 & 7446 & 7321 & 7454 & 10344 & 6737 & 7865 \\
\hline & 8751 & 7506 & 8536 & 11901 & 7289 & 9719 & 7417 & 7262 & 7472 & 10354 & 6748 & 7859 \\
\hline & 8481 & 7262 & 8183 & 11242 & 7136 & 9224 & 7845 & 7376 & 7673 & 10876 & 6907 & 8224 \\
\hline & 8010 & 6983 & 7764 & 10425 & 6975 & 8733 & 7987 & 7177 & 7712 & 10849 & 6830 & 8172 \\
\hline & 8022 & 7215 & 7915 & 10275 & 6993 & 8648 & 8208 & 7338 & 7770 & 11011 & 6930 & 8221 \\
\hline & 8119 & 7184 & 7902 & 10577 & 7086 & 8890 & 8260 & 7422 & 7806 & 11147 & 6933 & 8282 \\
\hline & 8536 & 7353 & 8160 & 11244 & 7155 & 9217 & 8154 & 7551 & 7816 & 11076 & 7011 & 8350 \\
\hline & 8969 & 7551 & 8546 & 11980 & 7340 & 9744 & 7641 & 7385 & 7554 & 10559 & 6829 & 7960 \\
\hline & 8671 & 7345 & 8186 & 11259 & 7165 & 9409 & 8026 & 7470 & 7770 & 10967 & 6949 & 8307 \\
\hline & 8141 & 7147 & 7841 & 10487 & 6972 & 8812 & 8206 & 7273 & 7731 & 10859 & 6884 & 8198 \\
\hline & 7865 & 6999 & 7692 & 9993 & 6833 & 8531 & 8303 & 7232 & 7630 & 10877 & 6949 & 8183 \\
\hline \multirow{14}{*}{ Control } & 8249 & 7163 & 8153 & 10825 & 6967 & 9025 & 8640 & 7373 & 7902 & 11425 & 6938 & 8354 \\
\hline & 8205 & 7212 & 8176 & 10875 & 7079 & 9086 & 8682 & 7395 & 7924 & 11566 & 6924 & 8542 \\
\hline & 8176 & 7219 & 8162 & 10935 & 7020 & 8942 & 8615 & 7361 & 8022 & 11601 & 6975 & 8533 \\
\hline & 8275 & 7195 & 8210 & 10976 & 7070 & 9141 & 8658 & 7398 & 7976 & 11591 & 6957 & 8593 \\
\hline & 8281 & 7203 & 8163 & 11107 & 7006 & 9184 & 8638 & 7492 & 8009 & 11697 & 6956 & 8639 \\
\hline & 8276 & 7246 & 8165 & 11265 & 7066 & 9261 & 8712 & 7485 & 8023 & 11808 & 7027 & 8645 \\
\hline & 8194 & 7254 & 8299 & 11422 & 7103 & 9319 & 8616 & 7431 & 8050 & 11908 & 7022 & 8782 \\
\hline & 8243 & 7195 & 8261 & 11330 & 7128 & 9371 & 8593 & 7477 & 8067 & 11940 & 7055 & 8765 \\
\hline & 8214 & 7235 & 8324 & 11376 & 7105 & 9361 & 8576 & 7504 & 8102 & 12014 & 7015 & 8818 \\
\hline & 8264 & 7287 & 8360 & 11516 & 7123 & 9449 & 8583 & 7524 & 8155 & 11978 & 7005 & 8784 \\
\hline & 8280 & 7281 & 8340 & 11458 & 7081 & 9395 & 8593 & 7572 & 8174 & 11925 & 7107 & 8818 \\
\hline & 8253 & 7296 & 8139 & 11122 & 7097 & 9289 & 8601 & 7458 & 8160 & 11811 & 7056 & 8699 \\
\hline & 8159 & 7194 & 8143 & 11135 & 7020 & 9220 & 8521 & 7434 & 8132 & 11798 & 7008 & 8666 \\
\hline & 8168 & 7234 & 8134 & 11154 & 7081 & 9207 & 8532 & 7471 & 8063 & 11699 & 7001 & 8674 \\
\hline \multirow{13}{*}{$\begin{array}{c}\text { Gloydius blomhoffi } \\
\text { siniticus }\end{array}$} & \multicolumn{12}{|c|}{ SRB } \\
\hline & $\mathrm{CH} 1$ & $\mathrm{CH} 2$ & $\mathrm{CH} 3$ & $\mathrm{CH} 4$ & CH5 & CH6 & CH1 & $\mathrm{CH} 2$ & $\mathrm{CH} 3$ & $\mathrm{CH} 4$ & CH5 & CH6 \\
\hline & 302 & 302 & 302 & 302 & 302 & 302 & 365 & 365 & 365 & 365 & 365 & 365 \\
\hline & 450 & 480 & 505 & 535 & 570 & 605 & 450 & 480 & 505 & 535 & 570 & 605 \\
\hline & 7426 & 6560 & 6252 & 6917 & 26017 & 30928 & 7778 & 6606 & 8221 & 8092 & 17692 & 20912 \\
\hline & 7348 & 6568 & 6283 & 6924 & 26244 & 31364 & 7687 & 6608 & 8272 & 8113 & 17734 & 21556 \\
\hline & 7385 & 6610 & 6286 & 6973 & 26665 & 32091 & 7681 & 6623 & 8259 & 8212 & 18086 & 21468 \\
\hline & 7405 & 6599 & 6245 & 7014 & 27214 & 32793 & 7743 & 6670 & 8210 & 8222 & 18432 & 21414 \\
\hline & 7402 & 6650 & 6268 & 6911 & 26987 & 32039 & 7707 & 6707 & 8295 & 8254 & 18334 & 22038 \\
\hline & 7492 & 6649 & 6251 & 7028 & 27280 & 32940 & 7702 & 6697 & 8365 & 8287 & 18479 & 22184 \\
\hline & 7426 & 6598 & 6269 & 7084 & 26821 & 32874 & 7686 & 6666 & 8190 & 8216 & 18294 & 22160 \\
\hline & 7343 & 6604 & 6263 & 7034 & 27850 & 33479 & 7683 & 6711 & 8217 & 8223 & 18732 & 22265 \\
\hline & 7425 & 6590 & 6269 & 7038 & 27827 & 33554 & 7743 & 6754 & 8316 & 8284 & 18782 & 22366 \\
\hline
\end{tabular}




\begin{tabular}{|c|c|c|c|c|c|c|c|c|c|c|c|c|}
\hline & 7357 & 6633 & 6251 & 7061 & 27232 & 32786 & 7679 & 6636 & 8275 & 8226 & 18640 & 22295 \\
\hline & 7369 & 6589 & 6263 & 7054 & 26592 & 32018 & 7668 & 6743 & 8392 & 8253 & 18475 & 21779 \\
\hline & 7371 & 6637 & 6249 & 6970 & 26721 & 32059 & 7673 & 6640 & 8256 & 8241 & 18358 & 21901 \\
\hline & 7344 & 6634 & 6252 & 7044 & 26369 & 31627 & 7713 & 6678 & 8323 & 8201 & 18182 & 21218 \\
\hline & 7347 & 6622 & 6270 & 6928 & 26044 & 30543 & 7678 & 6684 & 8207 & 8166 & 18253 & 20918 \\
\hline \multirow{14}{*}{$\begin{array}{c}\text { Bungarus } \\
\text { multicinctus }\end{array}$} & 7423 & 6572 & 6200 & 7010 & 26640 & 32641 & 7754 & 6599 & 8124 & 8141 & 17099 & 20683 \\
\hline & 7342 & 6561 & 6201 & 6966 & 26741 & 32805 & 7767 & 6628 & 8168 & 8134 & 17056 & 20445 \\
\hline & 7364 & 6548 & 6193 & 7017 & 26509 & 32584 & 7666 & 6702 & 8109 & 8193 & 17133 & 20355 \\
\hline & 7440 & 6569 & 6301 & 6982 & 26760 & 32777 & 7673 & 6628 & 8066 & 8201 & 17348 & 20908 \\
\hline & 7404 & 6518 & 6294 & 7114 & 26937 & 33500 & 7732 & 6698 & 8161 & 8222 & 17388 & 21325 \\
\hline & 7436 & 6570 & 6234 & 7071 & 27149 & 33588 & 7673 & 6656 & 8084 & 8230 & 17554 & 21546 \\
\hline & 7337 & 6521 & 6246 & 6942 & 26217 & 32989 & 7643 & 6615 & 8175 & 8245 & 17249 & 21012 \\
\hline & 7505 & 6547 & 6212 & 7168 & 28380 & 34994 & 7640 & 6758 & 8247 & 8301 & 18033 & 21830 \\
\hline & 7476 & 6615 & 6264 & 7135 & 27930 & 34592 & 7689 & 6673 & 8339 & 8291 & 17830 & 21310 \\
\hline & 7418 & 6596 & 6253 & 7165 & 28040 & 34536 & 7694 & 6676 & 8247 & 8316 & 17929 & 21789 \\
\hline & 7434 & 6575 & 6217 & 7026 & 27328 & 33421 & 7732 & 6687 & 8258 & 8282 & 17825 & 21576 \\
\hline & 7421 & 6561 & 6227 & 7103 & 27530 & 33589 & 7799 & 6674 & 8427 & 8272 & 17794 & 21107 \\
\hline & 7369 & 6514 & 6228 & 7036 & 27196 & 33093 & 7638 & 6649 & 8231 & 8225 & 17717 & 20924 \\
\hline & 7350 & 6586 & 6208 & 7042 & 26312 & 31735 & 7650 & 6618 & 8185 & 8113 & 17434 & 20419 \\
\hline \multirow{14}{*}{$\begin{array}{c}\text { Deinagkistrodon } \\
\text { acutus }\end{array}$} & 7775 & 6733 & 6286 & 7194 & 30866 & 37591 & 7699 & 6675 & 8067 & 8217 & 17640 & 20951 \\
\hline & 7790 & 6566 & 6420 & 7235 & 31431 & 38653 & 7707 & 6736 & 8017 & 8206 & 17506 & 21088 \\
\hline & 7781 & 6801 & 6309 & 7320 & 31557 & 38763 & 7707 & 6765 & 8113 & 8207 & 17695 & 21354 \\
\hline & 7753 & 6749 & 6346 & 7360 & 31855 & 39113 & 7640 & 6667 & 8170 & 8321 & 17883 & 21427 \\
\hline & 7802 & 6700 & 6318 & 7430 & 32270 & 39656 & 7674 & 6771 & 8065 & 8296 & 18046 & 21629 \\
\hline & 7748 & 6772 & 6294 & 7329 & 32148 & 39629 & 7710 & 6765 & 8195 & 8323 & 18279 & 21937 \\
\hline & 7712 & 6725 & 6381 & 7446 & 31764 & 39663 & 7682 & 6796 & 8101 & 8305 & 18044 & 21876 \\
\hline & 7801 & 6692 & 6309 & 7462 & 32088 & 39795 & 7719 & 6783 & 8199 & 8383 & 17940 & 21507 \\
\hline & 7742 & 6718 & 6332 & 7510 & 32007 & 39390 & 7717 & 6742 & 8230 & 8409 & 17849 & 21357 \\
\hline & 7804 & 6704 & 6417 & 7518 & 32257 & 39626 & 7697 & 6785 & 8216 & 8358 & 17970 & 21500 \\
\hline & 7759 & 6703 & 6267 & 7400 & 32765 & 39959 & 7699 & 6769 & 8122 & 8370 & 18368 & 21928 \\
\hline & 7712 & 6610 & 6379 & 7304 & 32039 & 38975 & 7575 & 6746 & 8140 & 8270 & 18019 & 21261 \\
\hline & 7711 & 6652 & 6303 & 7359 & 31814 & 38153 & 7672 & 6720 & 8166 & 8246 & 17913 & 21356 \\
\hline & 7729 & 6706 & 6323 & 7247 & 31805 & 38347 & 7601 & 6761 & 8150 & 8218 & 18150 & 21108 \\
\hline \multirow{8}{*}{$\begin{array}{c}\text { Daboia russelii } \\
\text { siamensis }\end{array}$} & 8381 & 6837 & 6386 & 7732 & 37265 & 45498 & 7475 & 6856 & 8270 & 8112 & 16981 & 19788 \\
\hline & 8291 & 6853 & 6335 & 7675 & 38019 & 46908 & 7498 & 6838 & 8247 & 8242 & 17086 & 20018 \\
\hline & 8277 & 6883 & 6440 & 7758 & 38502 & 47712 & 7505 & 6847 & 8384 & 8292 & 17492 & 20289 \\
\hline & 8280 & 6834 & 6436 & 7830 & 38857 & 47749 & 7549 & 6803 & 8420 & 8329 & 17312 & 20443 \\
\hline & 8320 & 6859 & 6475 & 7749 & 38846 & 47887 & 7440 & 6861 & 8398 & 8273 & 17299 & 20410 \\
\hline & 8206 & 6904 & 6369 & 7807 & 39029 & 47752 & 7513 & 6813 & 8390 & 8422 & 17491 & 20553 \\
\hline & 8253 & 6921 & 6422 & 7792 & 38610 & 48287 & 7453 & 6877 & 8330 & 8334 & 17514 & 20703 \\
\hline & 8313 & 6987 & 6399 & 7862 & 40129 & 49234 & 7568 & 6891 & 8386 & 8345 & 17723 & 20937 \\
\hline
\end{tabular}




\begin{tabular}{|c|c|c|c|c|c|c|c|c|c|c|c|c|}
\hline & 8230 & 6959 & 6478 & 7875 & 40201 & 49218 & 7518 & 6912 & 8365 & 8274 & 17801 & 20924 \\
\hline & 8267 & 6907 & 6429 & 7934 & 40357 & 49462 & 7509 & 6891 & 8406 & 8372 & 17698 & 20999 \\
\hline & 8246 & 6925 & 6477 & 7959 & 39148 & 48199 & 7392 & 6830 & 8466 & 8332 & 17374 & 20468 \\
\hline & 8213 & 6913 & 6423 & 7842 & 38345 & 47330 & 7442 & 6831 & 8323 & 8243 & 16969 & 20065 \\
\hline & 8214 & 6885 & 6479 & 7896 & 38627 & 47298 & 7440 & 6881 & 8403 & 8248 & 17059 & 20080 \\
\hline & 8153 & 6931 & 6427 & 7717 & 38473 & 46819 & 7404 & 6887 & 8348 & 8190 & 17151 & 19878 \\
\hline \multirow{14}{*}{ Naja atra } & 8641 & 6938 & 6441 & 8001 & 40938 & 49675 & 7087 & 6720 & 7940 & 8027 & 14506 & 16564 \\
\hline & 8726 & 7116 & 6402 & 7999 & 42447 & 53272 & 7051 & 6746 & 7899 & 8076 & 14649 & 17315 \\
\hline & 8687 & 6993 & 6447 & 8131 & 42701 & 52382 & 7090 & 6779 & 7932 & 8142 & 14795 & 17072 \\
\hline & 8660 & 6931 & 6495 & 8155 & 43551 & 53048 & 7104 & 6708 & 8023 & 8129 & 15092 & 17478 \\
\hline & 8659 & 7031 & 6479 & 8161 & 43288 & 52694 & 7113 & 6795 & 8091 & 8189 & 15053 & 17708 \\
\hline & 8675 & 7037 & 6489 & 8251 & 43826 & 53581 & 7116 & 6791 & 8090 & 8233 & 15227 & 18034 \\
\hline & 8558 & 6935 & 6460 & 8200 & 42435 & 52736 & 7115 & 6762 & 8019 & 8253 & 14940 & 17463 \\
\hline & 8681 & 7065 & 6488 & 8278 & 44662 & 54039 & 7164 & 6894 & 8101 & 8223 & 15390 & 18048 \\
\hline & 8687 & 7030 & 6520 & 8331 & 45190 & 54819 & 7157 & 6818 & 8041 & 8253 & 15347 & 18005 \\
\hline & 8589 & 7029 & 6488 & 8288 & 44290 & 53924 & 7097 & 6831 & 8042 & 8206 & 15112 & 17879 \\
\hline & 8665 & 7047 & 6549 & 8306 & 44603 & 55508 & 7281 & 6891 & 8347 & 8236 & 15225 & 17738 \\
\hline & 8616 & 7008 & 6509 & 8272 & 44391 & 53863 & 7090 & 6796 & 8004 & 8108 & 15039 & 17474 \\
\hline & 8479 & 7042 & 6488 & 8197 & 43930 & 53312 & 7116 & 6727 & 8038 & 8144 & 14869 & 17248 \\
\hline & 8475 & 7011 & 6493 & 8052 & 42744 & 51734 & 7097 & 6757 & 7977 & 8072 & 14734 & 16763 \\
\hline \multirow{14}{*}{ Agkistrodon Halys } & 8700 & 6898 & 6431 & 7843 & 39520 & 48983 & 7246 & 6704 & 8129 & 8220 & 15964 & 18820 \\
\hline & 8683 & 6992 & 6405 & 7914 & 40927 & 51267 & 7300 & 6733 & 8173 & 8305 & 16392 & 19217 \\
\hline & 8647 & 6974 & 6423 & 7983 & 39987 & 50330 & 7240 & 6765 & 8231 & 8271 & 16396 & 18754 \\
\hline & 8713 & 6991 & 6515 & 8149 & 41570 & 52328 & 7263 & 6772 & 8294 & 8356 & 16721 & 19780 \\
\hline & 8676 & 7023 & 6470 & 8021 & 41726 & 52256 & 7374 & 6826 & 8255 & 8346 & 16921 & 19611 \\
\hline & 8649 & 6995 & 6533 & 8188 & 41782 & 52409 & 7362 & 6827 & 8413 & 8409 & 16973 & 19775 \\
\hline & 8606 & 7058 & 6503 & 8054 & 40840 & 52086 & 7309 & 6853 & 8328 & 8388 & 16964 & 19681 \\
\hline & 8654 & 7030 & 6542 & 8185 & 42367 & 52871 & 7394 & 6907 & 8540 & 8447 & 17340 & 20190 \\
\hline & 8663 & 7015 & 6541 & 8211 & 43350 & 53889 & 7367 & 6881 & 8452 & 8460 & 17416 & 20214 \\
\hline & 8621 & 7000 & 6534 & 8126 & 42682 & 52975 & 7291 & 6853 & 8356 & 8410 & 17067 & 19966 \\
\hline & 8581 & 7057 & 6509 & 8145 & 42276 & 51659 & 7297 & 6870 & 8375 & 8338 & 17079 & 19771 \\
\hline & 8580 & 7086 & 6470 & 8055 & 42091 & 51679 & 7272 & 6822 & 8219 & 8297 & 16969 & 19612 \\
\hline & 8505 & 6981 & 6461 & 8021 & 41429 & 51340 & 7194 & 6771 & 8267 & 8305 & 16546 & 19142 \\
\hline & 8504 & 7030 & 6437 & 7924 & 40480 & 49828 & 7243 & 6820 & 8264 & 8220 & 16420 & 18883 \\
\hline \multirow{7}{*}{$\begin{array}{c}\text { Ophiophagus } \\
\text { hannah }\end{array}$} & 8258 & 6888 & 6485 & 7687 & 35071 & 44325 & 7907 & 6854 & 9518 & 8475 & 18308 & 22130 \\
\hline & 8260 & 6852 & 6350 & 7634 & 36082 & 46141 & 7819 & 6895 & 9544 & 8569 & 18658 & 22513 \\
\hline & 8262 & 6954 & 6396 & 7710 & 36540 & 46912 & 7897 & 6948 & 9658 & 8691 & 19155 & 22863 \\
\hline & 8251 & 6937 & 6519 & 7748 & 36969 & 47126 & 7857 & 6947 & 9737 & 8659 & 19347 & 23208 \\
\hline & 8277 & 6927 & 6451 & 7809 & 36525 & 46841 & 7856 & 6990 & 9734 & 8729 & 19128 & 23113 \\
\hline & 8393 & 6958 & 6486 & 7925 & 37292 & 47964 & 7894 & 6987 & 9837 & 8752 & 19454 & 23494 \\
\hline & 8254 & 6976 & 6454 & 7896 & 35946 & 46968 & 7876 & 7008 & 9812 & 8851 & 19313 & 23313 \\
\hline
\end{tabular}




\begin{tabular}{|c|c|c|c|c|c|c|c|c|c|c|c|c|}
\hline & 8317 & 6976 & 6456 & 7833 & 37582 & 47690 & 7922 & 7029 & 9870 & 8764 & 19985 & 23822 \\
\hline & 8438 & 7026 & 6521 & 7888 & 38069 & 47976 & 7886 & 7005 & 9866 & 8846 & 19845 & 23929 \\
\hline & 8294 & 6968 & 6511 & 7864 & 37572 & 47473 & 7887 & 7040 & 9861 & 8834 & 19694 & 23708 \\
\hline & 8320 & 6983 & 6471 & 7827 & 37838 & 47174 & 7844 & 7059 & 9805 & 8763 & 20014 & 23860 \\
\hline & 8235 & 6912 & 6426 & 7750 & 37337 & 46114 & 7829 & 6986 & 9847 & 8637 & 19731 & 23364 \\
\hline & 8145 & 6960 & 6426 & 7706 & 36969 & 45773 & 7804 & 6913 & 9715 & 8624 & 19362 & 23007 \\
\hline & 8218 & 7042 & 6440 & 7600 & 35848 & 44322 & 8202 & 7173 & 10509 & 8526 & 19018 & 22361 \\
\hline \multirow{14}{*}{ BSA } & 7246 & 6512 & 6248 & 6903 & 25145 & 28955 & 7596 & 6637 & 8294 & 8020 & 17603 & 20206 \\
\hline & 7309 & 6529 & 6177 & 7003 & 26138 & 30511 & 7576 & 6636 & 8214 & 8043 & 17126 & 19928 \\
\hline & 7598 & 6681 & 6332 & 7254 & 30946 & 37072 & 7599 & 6723 & 8321 & 8144 & 17347 & 20155 \\
\hline & 8197 & 6894 & 6460 & 7657 & 38592 & 46661 & 7380 & 6741 & 8184 & 8140 & 16768 & 19224 \\
\hline & 8376 & 6981 & 6434 & 7961 & 41753 & 51348 & 7198 & 6785 & 8243 & 8203 & 16484 & 18824 \\
\hline & 7997 & 6859 & 6422 & 7567 & 35947 & 43728 & 7583 & 6848 & 8428 & 8278 & 18503 & 21606 \\
\hline & 7640 & 6794 & 6446 & 7388 & 33046 & 39425 & 7708 & 6791 & 8367 & 8305 & 19043 & 22199 \\
\hline & 7302 & 6519 & 6220 & 6879 & 25190 & 28943 & 7654 & 6681 & 8151 & 8017 & 17598 & 19490 \\
\hline & 7289 & 6484 & 6221 & 6915 & 26041 & 30227 & 7638 & 6623 & 8166 & 8016 & 16971 & 19537 \\
\hline & 7612 & 6646 & 6314 & 7178 & 30398 & 35624 & 7519 & 6707 & 8198 & 8052 & 17127 & 19476 \\
\hline & 8162 & 6801 & 6437 & 7581 & 37543 & 45227 & 7531 & 6842 & 8558 & 8091 & 16508 & 18693 \\
\hline & 8437 & 6924 & 6408 & 7816 & 40858 & 49585 & 7231 & 6829 & 8234 & 8123 & 16189 & 18445 \\
\hline & 7956 & 6795 & 6385 & 7454 & 35640 & 43108 & 7665 & 6893 & 8407 & 8217 & 18512 & 21423 \\
\hline & 7574 & 6650 & 6327 & 7191 & 32381 & 38703 & 7688 & 6766 & 8181 & 8233 & 18921 & 21925 \\
\hline \multirow{14}{*}{ Thrombin } & 8009 & 6202 & 5302 & 3982 & 17018 & 45440 & 8811 & 6217 & 11476 & 7660 & 10971 & 14465 \\
\hline & 7987 & 6513 & 5563 & 4145 & 16944 & 44086 & 9148 & 6043 & 10535 & 7637 & 9676 & 14025 \\
\hline & 8203 & 5921 & 5326 & 4435 & 17761 & 43636 & 8045 & 6768 & 10200 & 6586 & 9717 & 14525 \\
\hline & 7212 & 6217 & 5492 & 4531 & 17751 & 41231 & 8392 & 7639 & 11877 & 8113 & 10122 & 13803 \\
\hline & 7036 & 6733 & 5690 & 4783 & 16982 & 40579 & 7716 & 6739 & 10854 & 7964 & 10428 & 13315 \\
\hline & 8032 & 6880 & 6737 & 4788 & 16028 & 40460 & 7547 & 6769 & 10342 & 6446 & 9461 & 13467 \\
\hline & 7163 & 6923 & 6196 & 4725 & 19437 & 36500 & 7775 & 7073 & 9800 & 6963 & 9845 & 14148 \\
\hline & 7997 & 6874 & 6748 & 5092 & 19979 & 34752 & 8440 & 7027 & 10672 & 7859 & 9552 & 14730 \\
\hline & 7879 & 6454 & 6190 & 5347 & 18828 & 41045 & 8852 & 7260 & 11337 & 7181 & 11159 & 15063 \\
\hline & 8100 & 6228 & 7021 & 5406 & 19181 & 45916 & 9063 & 7607 & 11480 & 6997 & 10323 & 14359 \\
\hline & 7685 & 5810 & 6123 & 5198 & 17239 & 40442 & 8893 & 7689 & 11247 & 6473 & 9476 & 13835 \\
\hline & 8344 & 7031 & 6730 & 4918 & 18458 & 39212 & 8713 & 7707 & 11811 & 6852 & 9804 & 13357 \\
\hline & 7617 & 5928 & 5466 & 4972 & 17063 & 44923 & 8514 & 6605 & 11689 & 6729 & 9637 & 13480 \\
\hline & 7692 & 6527 & 6228 & 4777 & 20260 & 44808 & 9813 & 5972 & 11352 & 6684 & 11092 & 14110 \\
\hline \multirow{7}{*}{ TRF } & 7198 & 6467 & 6172 & 6733 & 21819 & 24667 & 7476 & 6581 & 7852 & 7918 & 15983 & 18128 \\
\hline & 7153 & 6448 & 6231 & 6790 & 22672 & 26316 & 7496 & 6530 & 7806 & 7973 & 15697 & 18023 \\
\hline & 7407 & 6586 & 6352 & 7107 & 25991 & 30589 & 7473 & 6542 & 7859 & 8009 & 15645 & 17941 \\
\hline & 7974 & 6662 & 6270 & 7346 & 33041 & 39154 & 7228 & 6686 & 7717 & 7928 & 15599 & 17782 \\
\hline & 8035 & 6803 & 6319 & 7434 & 35223 & 41501 & 7068 & 6640 & 7711 & 7960 & 15067 & 17026 \\
\hline & 7737 & 6659 & 6257 & 7226 & 31027 & 36412 & 7436 & 6718 & 7890 & 8098 & 17250 & 19777 \\
\hline & 7388 & 6599 & 6284 & 6951 & 28629 & 33325 & 7524 & 6667 & 7872 & 8084 & 17723 & 20359 \\
\hline
\end{tabular}




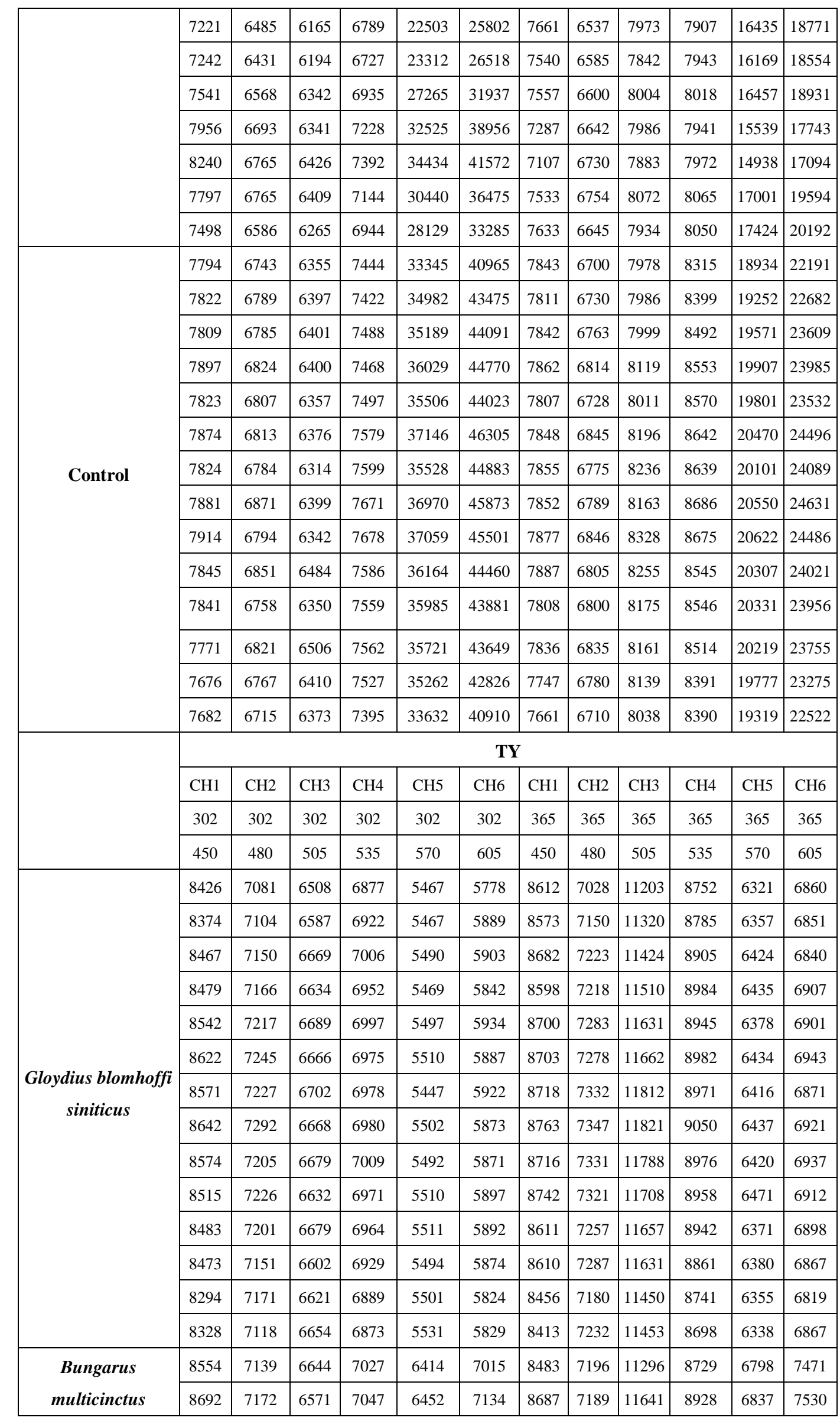




\begin{tabular}{|c|c|c|c|c|c|c|c|c|c|c|c|c|}
\hline & 8618 & 7193 & 6659 & 7114 & 6495 & 7165 & 8585 & 7342 & 11632 & 9014 & 6876 & 7529 \\
\hline & 8674 & 7220 & 6667 & 7073 & 6446 & 7159 & 8654 & 7254 & 11770 & 9052 & 6910 & 7621 \\
\hline & 8758 & 7267 & 6745 & 7107 & 6464 & 7202 & 8727 & 7354 & 12064 & 9122 & 6949 & 7674 \\
\hline & 8874 & 7290 & 6695 & 7118 & 6497 & 7190 & 8774 & 7372 & 12076 & 9105 & 6960 & 7679 \\
\hline & 8770 & 7302 & 6684 & 7154 & 6505 & 7234 & 8775 & 7376 & 12072 & 9158 & 6988 & 7711 \\
\hline & 8901 & 7353 & 6687 & 7182 & 6550 & 7229 & 8851 & 7437 & 12182 & 9204 & 6967 & 7810 \\
\hline & 8877 & 7338 & 6756 & 7178 & 6570 & 7289 & 8796 & 7406 & 12239 & 9170 & 7014 & 7770 \\
\hline & 8749 & 7304 & 6670 & 7119 & 6499 & 7246 & 8698 & 7419 & 12129 & 9099 & 6958 & 7693 \\
\hline & 8769 & 7310 & 6701 & 7154 & 6535 & 7192 & 8753 & 7408 & 12309 & 9125 & 6947 & 7718 \\
\hline & 8787 & 7266 & 6690 & 7088 & 6561 & 7132 & 8663 & 7371 & 11990 & 9043 & 6948 & 7655 \\
\hline & 8604 & 7181 & 6664 & 7017 & 6424 & 7137 & 8523 & 7310 & 11721 & 8915 & 6867 & 7552 \\
\hline & 8554 & 7152 & 6628 & 7033 & 6464 & 7067 & 8434 & 7284 & 11687 & 8826 & 6868 & 7525 \\
\hline \multirow{14}{*}{$\begin{array}{c}\text { Deinagkistrodon } \\
\text { acutus }\end{array}$} & 9405 & 7389 & 6723 & 7137 & 6532 & 7239 & 8055 & 7161 & 10320 & 8589 & 6654 & 7117 \\
\hline & 9446 & 7381 & 6659 & 7112 & 6611 & 7316 & 8095 & 7127 & 10390 & 8638 & 6679 & 7177 \\
\hline & 9435 & 7455 & 6734 & 7142 & 6561 & 7294 & 8093 & 7176 & 10523 & 8691 & 6651 & 7218 \\
\hline & 9453 & 7498 & 6790 & 7162 & 6715 & 7355 & 8084 & 7215 & 10651 & 8728 & 6686 & 7205 \\
\hline & 9563 & 7483 & 6767 & 7210 & 6613 & 7373 & 8199 & 7346 & 10710 & 8854 & 6750 & 7311 \\
\hline & 9608 & 7546 & 6843 & 7150 & 6571 & 7431 & 8257 & 7332 & 10727 & 8793 & 6824 & 7255 \\
\hline & 9610 & 7563 & 6814 & 7243 & 6735 & 7454 & 8186 & 7321 & 10941 & 8889 & 6731 & 7298 \\
\hline & 9574 & 7579 & 6781 & 7193 & 6679 & 7447 & 8250 & 7302 & 10903 & 8876 & 6733 & 7339 \\
\hline & 9631 & 7587 & 6895 & 7207 & 6645 & 7467 & 8276 & 7411 & 10984 & 8881 & 6740 & 7322 \\
\hline & 9541 & 7572 & 6823 & 7138 & 6653 & 7384 & 8164 & 7278 & 10885 & 8808 & 6736 & 7245 \\
\hline & 9437 & 7537 & 6810 & 7188 & 6630 & 7330 & 8144 & 7332 & 10813 & 8737 & 6761 & 7274 \\
\hline & 9450 & 7485 & 6759 & 7163 & 6566 & 7344 & 8084 & 7252 & 10728 & 8700 & 6719 & 7188 \\
\hline & 9276 & 7467 & 6760 & 7153 & 6646 & 7269 & 7995 & 7169 & 10642 & 8652 & 6642 & 7155 \\
\hline & 9225 & 7371 & 6736 & 7062 & 6564 & 7233 & 7879 & 7234 & 10490 & 8530 & 6624 & 7194 \\
\hline \multirow{14}{*}{$\begin{array}{c}\text { Daboia russelii } \\
\text { siamensis }\end{array}$} & 9825 & 7567 & 6783 & 7425 & 6856 & 7807 & 7800 & 7194 & 11268 & 8862 & 6860 & 7518 \\
\hline & 9757 & 7575 & 6854 & 7426 & 6852 & 7751 & 7687 & 7217 & 11331 & 8858 & 6821 & 7478 \\
\hline & 9859 & 7712 & 6883 & 7459 & 6925 & 7858 & 7743 & 7188 & 11692 & 8967 & 6910 & 7593 \\
\hline & 9912 & 7611 & 6905 & 7510 & 6876 & 7972 & 7880 & 7247 & 11584 & 9024 & 6924 & 7628 \\
\hline & 10004 & 7681 & 6970 & 7545 & 6872 & 7893 & 7907 & 7377 & 11758 & 9054 & 6939 & 7648 \\
\hline & 9916 & 7767 & 6904 & 7536 & 6848 & 7972 & 7886 & 7341 & 11715 & 9048 & 6950 & 7655 \\
\hline & 9816 & 7685 & 6920 & 7524 & 6909 & 7929 & 7904 & 7332 & 11896 & 9056 & 6975 & 7613 \\
\hline & 9785 & 7682 & 6870 & 7452 & 6891 & 7870 & 7949 & 7358 & 11784 & 9032 & 6912 & 7695 \\
\hline & 9732 & 7735 & 6885 & 7596 & 6943 & 7895 & 7849 & 7352 & 11829 & 9036 & 6932 & 7627 \\
\hline & 9615 & 7611 & 6842 & 7440 & 6875 & 7735 & 7767 & 7240 & 11498 & 8925 & 6910 & 7529 \\
\hline & 9678 & 7673 & 6876 & 7533 & 6902 & 7907 & 7801 & 7387 & 11807 & 8947 & 6932 & 7606 \\
\hline & 9496 & 7623 & 6937 & 7486 & 6891 & 7799 & 7786 & 7281 & 11774 & 8927 & 6923 & 7614 \\
\hline & 9478 & 7534 & 6817 & 7307 & 6826 & 7729 & 7729 & 7155 & 11337 & 8764 & 6902 & 7470 \\
\hline & 9450 & 7503 & 6882 & 7417 & 6795 & 7715 & 7638 & 7204 & 11268 & 8752 & 6942 & 7485 \\
\hline Naja atra & 9570 & 7463 & 6764 & 7207 & 6601 & 7254 & 8062 & 7347 & 12595 & 8696 & 6634 & 7177 \\
\hline
\end{tabular}




\begin{tabular}{|c|c|c|c|c|c|c|c|c|c|c|c|c|}
\hline & 9607 & 7539 & 6744 & 7210 & 6637 & 7364 & 8184 & 7376 & 12807 & 8781 & 6641 & 7216 \\
\hline & 9656 & 7664 & 6800 & 7295 & 6646 & 7419 & 8199 & 7531 & 12887 & 8841 & 6665 & 7136 \\
\hline & 9731 & 7561 & 6809 & 7233 & 6724 & 7391 & 8229 & 7457 & 13153 & 8890 & 6672 & 7232 \\
\hline & 9688 & 7650 & 6856 & 7313 & 6696 & 7411 & 8343 & 7542 & 13209 & 8921 & 6755 & 7245 \\
\hline & 9841 & 7671 & 6868 & 7286 & 6741 & 7400 & 8420 & 7593 & 13367 & 8964 & 6758 & 7281 \\
\hline & 9724 & 7682 & 6849 & 7320 & 6716 & 7440 & 8362 & 7601 & 13150 & 8995 & 6736 & 7257 \\
\hline & 9669 & 7715 & 6822 & 7348 & 6682 & 7377 & 8389 & 7619 & 13246 & 8982 & 6685 & 7331 \\
\hline & 9634 & 7672 & 6820 & 7251 & 6649 & 7377 & 8257 & 7476 & 13241 & 8852 & 6709 & 7242 \\
\hline & 9489 & 7604 & 6872 & 7225 & 6566 & 7396 & 8220 & 7549 & 13157 & 8854 & 6672 & 7229 \\
\hline & 9402 & 7563 & 6808 & 7217 & 6648 & 7326 & 8158 & 7470 & 13097 & 8780 & 6625 & 7246 \\
\hline & 9341 & 7533 & 6810 & 7211 & 6588 & 7290 & 8129 & 7485 & 13098 & 8767 & 6673 & 7160 \\
\hline & 9292 & 7524 & 6808 & 7121 & 6618 & 7249 & 8097 & 7425 & 12830 & 8719 & 6673 & 7191 \\
\hline & 9347 & 7531 & 6850 & 7169 & 6620 & 7279 & 8097 & 7448 & 12812 & 8732 & 6681 & 7140 \\
\hline \multirow{14}{*}{ Agkistrodon Halys } & 9048 & 7268 & 6698 & 7043 & 6423 & 7102 & 9063 & 7383 & 12816 & 8746 & 6690 & 7254 \\
\hline & 8744 & 7179 & 6598 & 6942 & 6510 & 7118 & 8436 & 7166 & 11033 & 8703 & 6667 & 7268 \\
\hline & 8741 & 7242 & 6643 & 7023 & 6508 & 7146 & 8416 & 7265 & 11162 & 8769 & 6780 & 7278 \\
\hline & 8724 & 7172 & 6587 & 7008 & 6508 & 7122 & 8371 & 7145 & 11224 & 8753 & 6676 & 7271 \\
\hline & 8763 & 7247 & 6681 & 6988 & 6581 & 7135 & 8505 & 7248 & 11406 & 8855 & 6775 & 7323 \\
\hline & 8876 & 7215 & 6654 & 7005 & 6543 & 7209 & 8469 & 7290 & 11328 & 8789 & 6826 & 7345 \\
\hline & 8728 & 7224 & 6616 & 7004 & 6564 & 7181 & 8486 & 7248 & 11315 & 8808 & 6790 & 7318 \\
\hline & 8802 & 7231 & 6692 & 7019 & 6570 & 7185 & 8494 & 7343 & 11452 & 8865 & 6833 & 7396 \\
\hline & 8813 & 7219 & 6615 & 7039 & 6592 & 7193 & 8535 & 7322 & 11606 & 8841 & 6738 & 7357 \\
\hline & 8710 & 7125 & 6643 & 7028 & 6527 & 7144 & 8443 & 7265 & 11430 & 8764 & 6782 & 7386 \\
\hline & 8668 & 7287 & 6659 & 6967 & 6574 & 7113 & 8424 & 7225 & 11340 & 8733 & 6764 & 7375 \\
\hline & 8653 & 7185 & 6611 & 6994 & 6533 & 7181 & 8330 & 7206 & 11337 & 8690 & 6729 & 7285 \\
\hline & 8526 & 7121 & 6563 & 6935 & 6485 & 7110 & 8272 & 7235 & 11288 & 8696 & 6705 & 7219 \\
\hline & 8513 & 7153 & 6619 & 6931 & 6483 & 7110 & 8277 & 7194 & 11157 & 8630 & 6701 & 7227 \\
\hline \multirow{14}{*}{$\begin{array}{c}\text { Ophiophagus } \\
\text { hannah }\end{array}$} & 8384 & 7032 & 6500 & 6930 & 6550 & 7140 & 8664 & 7131 & 12941 & 8958 & 6919 & 7716 \\
\hline & 8366 & 7022 & 6528 & 6953 & 6520 & 7183 & 8691 & 7135 & 13116 & 9010 & 6898 & 7774 \\
\hline & 8421 & 7030 & 6560 & 6989 & 6513 & 7205 & 8758 & 7302 & 13229 & 9090 & 6968 & 7827 \\
\hline & 8381 & 7066 & 6633 & 6890 & 6579 & 7211 & 8631 & 7295 & 13265 & 9095 & 6959 & 7829 \\
\hline & 8374 & 7138 & 6543 & 6971 & 6626 & 7236 & 8695 & 7306 & 13393 & 9116 & 6952 & 7782 \\
\hline & 8506 & 7110 & 6611 & 7064 & 6575 & 7260 & 8808 & 7355 & 13592 & 9200 & 7026 & 7876 \\
\hline & 8433 & 7039 & 6625 & 7024 & 6516 & 7251 & 8748 & 7347 & 13306 & 9187 & 6964 & 7849 \\
\hline & 8441 & 7101 & 6586 & 6996 & 6587 & 7243 & 8678 & 7314 & 13369 & 9137 & 7066 & 7849 \\
\hline & 8430 & 7077 & 6625 & 6973 & 6546 & 7269 & 8732 & 7368 & 13418 & 9170 & 7001 & 7835 \\
\hline & 8406 & 7061 & 6552 & 6986 & 6558 & 7170 & 8588 & 7289 & 13363 & 9154 & 6995 & 7779 \\
\hline & 8420 & 7081 & 6577 & 7032 & 6543 & 7168 & 8561 & 7301 & 13292 & 9033 & 7032 & 7809 \\
\hline & 8346 & 7049 & 6556 & 6931 & 6541 & 7181 & 8555 & 7232 & 13370 & 9093 & 6947 & 7831 \\
\hline & 8216 & 7032 & 6575 & 6860 & 6517 & 7178 & 8536 & 7292 & 13321 & 9065 & 7002 & 7763 \\
\hline & 8242 & 7039 & 6597 & 6904 & 6567 & 7155 & 8464 & 7188 & 13146 & 8944 & 6967 & 7682 \\
\hline BSA & 8738 & 7403 & 6783 & 7043 & 6236 & 6863 & 8963 & 7811 & 15410 & 8834 & 6586 & 7114 \\
\hline
\end{tabular}




\begin{tabular}{|c|c|c|c|c|c|c|c|c|c|c|c|c|}
\hline & 9196 & 7512 & 6917 & 7210 & 6535 & 7286 & 8429 & 7688 & 14174 & 8741 & 6641 & 7155 \\
\hline & 9587 & 7603 & 6954 & 7379 & 6545 & 7416 & 7782 & 7418 & 12879 & 8610 & 6598 & 6965 \\
\hline & 9199 & 7521 & 6817 & 7141 & 6571 & 7192 & 8181 & 7507 & 13886 & 8727 & 6561 & 7097 \\
\hline & 8528 & 7239 & 6697 & 6992 & 6468 & 7040 & 8812 & 7624 & 14707 & 8669 & 6634 & 7108 \\
\hline & 8151 & 7051 & 6602 & 6871 & 6379 & 6962 & 8775 & 7585 & 14859 & 8628 & 6588 & 7115 \\
\hline & 8126 & 7059 & 6674 & 6895 & 6480 & 6969 & 8986 & 7628 & 15005 & 8713 & 6637 & 7145 \\
\hline & 8487 & 7269 & 6740 & 6973 & 6287 & 6921 & 8866 & 7801 & 14931 & 8789 & 6575 & 7125 \\
\hline & 9144 & 7500 & 6822 & 7144 & 6523 & 7234 & 8334 & 7630 & 13827 & 8744 & 6640 & 7152 \\
\hline & 9355 & 7598 & 6884 & 7243 & 6568 & 7292 & 7762 & 7432 & 12489 & 8570 & 6603 & 6953 \\
\hline & 9077 & 7437 & 6804 & 7176 & 6606 & 7303 & 8170 & 7568 & 13588 & 8700 & 6663 & 7122 \\
\hline & 8508 & 7252 & 6712 & 6936 & 6478 & 7024 & 8724 & 7639 & 14387 & 8659 & 6592 & 7072 \\
\hline & 8139 & 7008 & 6637 & 6912 & 6401 & 6860 & 8780 & 7581 & 14363 & 8631 & 6610 & 7080 \\
\hline & 8014 & 6963 & 6583 & 6817 & 6439 & 6965 & 8838 & 7571 & 14740 & 8678 & 6594 & 7188 \\
\hline \multirow{14}{*}{ Thrombin } & 7382 & 6230 & 4704 & 7843 & 5703 & 5629 & 6206 & 4561 & 8411 & 6725 & 6225 & 5960 \\
\hline & 6650 & 6393 & 4863 & 7294 & 5940 & 5709 & 6086 & 4015 & 8637 & 7229 & 5955 & 5723 \\
\hline & 6720 & 6094 & 4962 & 7410 & 6077 & 5854 & 6204 & 4161 & 7661 & 7192 & 6666 & 5681 \\
\hline & 7133 & 6098 & 4809 & 6829 & 5892 & 6187 & 6013 & 4302 & 8471 & 7255 & 5871 & 6075 \\
\hline & 7094 & 6192 & 4757 & 7655 & 7012 & 5933 & 6156 & 4314 & 8846 & 7720 & 6703 & 6381 \\
\hline & 7079 & 7069 & 4618 & 7781 & 6903 & 5783 & 5919 & 4284 & 8158 & 8147 & 6840 & 7003 \\
\hline & 7485 & 7703 & 4612 & 7188 & 6500 & 5916 & 6276 & 4517 & 8619 & 7565 & 6511 & 7082 \\
\hline & 6750 & 7211 & 4992 & 7806 & 6374 & 5688 & 6348 & 4574 & 8498 & 7715 & 6281 & 6937 \\
\hline & 6945 & 8436 & 4939 & 7012 & 6639 & 5830 & 6181 & 4678 & 8159 & 7510 & 6035 & 6768 \\
\hline & 7786 & 6943 & 5141 & 7938 & 5532 & 5920 & 5630 & 4260 & 8286 & 7154 & 5682 & 6522 \\
\hline & 6651 & 6116 & 5143 & 6900 & 6496 & 6323 & 6006 & 4189 & 8422 & 7161 & 6005 & 6520 \\
\hline & 6941 & 7305 & 4634 & 7304 & 5535 & 5522 & 5074 & 4244 & 7386 & 7230 & 6473 & 5962 \\
\hline & 6546 & 6499 & 4549 & 7202 & 5519 & 6362 & 5255 & 4260 & 7236 & 7118 & 5869 & 5997 \\
\hline & 6744 & 7206 & 4413 & 7094 & 5564 & 5687 & 5376 & 3859 & 8255 & 6665 & 5766 & 6492 \\
\hline \multirow{14}{*}{ TRF } & 8202 & 6998 & 6542 & 6719 & 6263 & 6663 & 8149 & 7002 & 10147 & 8321 & 6482 & 6827 \\
\hline & 8616 & 7112 & 6601 & 6832 & 6368 & 6884 & 7635 & 6898 & 9686 & 8233 & 6463 & 6815 \\
\hline & 8885 & 7198 & 6677 & 6943 & 6444 & 6954 & 7246 & 6871 & 9186 & 8128 & 6444 & 6724 \\
\hline & 8700 & 7076 & 6633 & 6851 & 6490 & 6929 & 7528 & 6954 & 9497 & 8197 & 6500 & 6766 \\
\hline & 8097 & 6933 & 6468 & 6635 & 6327 & 6797 & 7900 & 6912 & 9791 & 8223 & 6472 & 6747 \\
\hline & 7822 & 6832 & 6405 & 6671 & 6277 & 6744 & 8029 & 6824 & 9810 & 8144 & 6535 & 6767 \\
\hline & 7752 & 6796 & 6450 & 6680 & 6385 & 6729 & 8180 & 6951 & 10050 & 8151 & 6480 & 6832 \\
\hline & 8364 & 7009 & 6536 & 6777 & 6287 & 6753 & 8217 & 7143 & 10467 & 8305 & 6468 & 6851 \\
\hline & 8889 & 7208 & 6682 & 6918 & 6558 & 6983 & 7756 & 7033 & 9999 & 8281 & 6572 & 6905 \\
\hline & 9103 & 7286 & 6676 & 6945 & 6470 & 7046 & 7347 & 6939 & 9595 & 8225 & 6560 & 6818 \\
\hline & 8935 & 7193 & 6628 & 6883 & 6460 & 7039 & 7723 & 7086 & 9956 & 8262 & 6536 & 6796 \\
\hline & 8374 & 7002 & 6490 & 6772 & 6398 & 6853 & 8093 & 7060 & 10320 & 8264 & 6568 & 6919 \\
\hline & 7944 & 6839 & 6412 & 6665 & 6358 & 6705 & 8146 & 6982 & 10231 & 8205 & 6540 & 6840 \\
\hline & 7909 & 6838 & 6505 & 6647 & 6439 & 6767 & 8317 & 6971 & 10699 & 8193 & 6505 & 6906 \\
\hline Control & 8289 & 7068 & 6419 & 6742 & 6404 & 6794 & 8803 & 7167 & 10684 & 8276 & 6463 & 6771 \\
\hline
\end{tabular}




\begin{tabular}{|l|l|l|l|l|l|l|l|l|l|l|l|l|}
\hline & 8173 & 6981 & 6525 & 6748 & 6401 & 6843 & 8577 & 7071 & 10234 & 8324 & 6457 & 6806 \\
\hline 8125 & 6968 & 6566 & 6725 & 6368 & 6785 & 8547 & 7173 & 10277 & 8344 & 6482 & 6792 \\
\hline 8186 & 7031 & 6431 & 6775 & 6357 & 6835 & 8553 & 7100 & 10303 & 8412 & 6496 & 6841 \\
\hline 8252 & 6990 & 6520 & 6750 & 6340 & 6869 & 8535 & 7139 & 10387 & 8424 & 6480 & 6851 \\
\hline 8234 & 7034 & 6496 & 6791 & 6406 & 6914 & 8517 & 7127 & 10344 & 8362 & 6458 & 6847 \\
\hline 8231 & 7042 & 6589 & 6780 & 6390 & 6939 & 8533 & 7172 & 10499 & 8432 & 6572 & 6857 \\
\hline 8205 & 7067 & 6482 & 6764 & 6363 & 6862 & 8611 & 7156 & 10505 & 8487 & 6484 & 6858 \\
\hline 8200 & 7065 & 6453 & 6737 & 6411 & 6836 & 8496 & 7159 & 10465 & 8395 & 6518 & 6858 \\
\hline 8157 & 6992 & 6494 & 6750 & 6356 & 6812 & 8502 & 7165 & 10523 & 8372 & 6495 & 6853 \\
\hline 8137 & 7000 & 6542 & 6803 & 6342 & 6832 & 8527 & 7198 & 10684 & 8445 & 6480 & 6817 \\
\hline 8089 & 6960 & 6540 & 6792 & 6349 & 6773 & 8460 & 7139 & 10482 & 8341 & 6509 & 6848 \\
\hline 8055 & 7051 & 6493 & 6783 & 6375 & 6801 & 8469 & 7095 & 10376 & 8300 & 6441 & 6812 \\
\hline 8004 & 6973 & 6500 & 6639 & 6337 & 6764 & 8400 & 7042 & 10155 & 8264 & 6473 & 6758 \\
\hline
\end{tabular}

89 
(1) Otto, M., Chemometrics: Statistics and Computer Application in Analytical Chemistry. Wiley-VCH 1999.

92 (2) Jambu, M., In Exploratory and MultiVariate Data Analysis. Academic Press: 1991.

93

(3) Huang, Y.; Li, F.; Qin, M.; Jiang, L.; Song, Y., A multi-stopband photonic-crystal microchip for high-

94 performance metal-ion recognition based on fluorescent detection. Angew. Chem. Int. Ed. Engl. 2013, 52 (28), 7296- 